\title{
Colloidal Polymers from Inorganic Nanoparticle Monomers
}

Lawrence J. Hill, ${ }^{\mathrm{a}}$ Nicola Pinna, ${ }^{\mathrm{b}}$ Kookheon Char, ${ }^{\mathrm{c}}$ and Jeffrey Pyun ${ }^{\mathrm{a}, \mathrm{c}^{*}}$

a Department of Chemistry and Biochemistry, University of Arizona, 1306 E. University Blvd., Tucson, AZ 85721, USA,

${ }^{\mathrm{b}}$ Institut für Chemie, Humboldt-Universität zu Berlin, Brook-Taylor-Straße 2, 12489 Berlin, Germany,

${ }^{c}$ World Class University Program for Chemical Convergence for Energy and Environment, The National Creative Research Initiative Center for Intelligent Hybrids, School of Chemical and Biological Engineering, Seoul National University, Seoul 151-744, Korea.

\begin{abstract}
The use of preformed inorganic nanoparticles as "colloidal monomers" has received recent attention for the formation of one-dimensional (1-D) mesostructures, or "colloidal polymers." These colloidal monomers form linear assemblies through attractive, directional, interparticle interactions, which are similar to covalent or supramolecular interactions in classical polymer science. However, in contrast to the high degree of structural control available in the synthesis of classical molecular polymers, methods to control fundamental structural features such as chain length (DP), composition (copolymers), and architecture (linear, branched, etc.) are still being developed for NP-based colloidal polymer systems. We therefore review the colloidal polymerization of inorganic nanoparticle monomers by applying the conceptual framework provided by polymer science to categorize these novel systems. The descriptive nomenclature used for classical polymers is applied to NP assembly to define more explicitly the types of colloidal polymers formed in terms of DP, architecture, and composition (for binary NP assemblies). This Review includes descriptions of inorganic nanoparticle types useful for the formation of colloidal polymers with examples chosen to demonstrate control over mesoscopic structure and composition. The various emergent optical, electrical and electrochemical properties from these materials are also reviewed and correlated with structural control achieved in various colloidal polymer systems.
\end{abstract}

Keywords: Colloidal polymer, nanopolymer, nanoparticle assembly, colloidal polymerization

Corresponding Author: jpyun@email.arizona.edu 


\section{Table of Contents}

1. Introduction

1.1 Types of colloidal polymerizations

1.2 Synthesis of colloidal monomers

2. Ligand-directed colloidal polymerization

2.1 Ligand induced electrostatic charge

2.2 Multifunctional ligands

2.3 Non-covalent ligand interactions

3. Surface-directed colloidal polymerization

3.1 Oriented attachment

3.2 Nanowelding

4. Dipole-directed colloidal polymerization

4.1 Electrostatic dipoles

4.2 Magnetic dipoles

5. Properties of colloidal polymers

5.1 Properties correlated with DP, monomer spacing, and monomer orientation

5.2 Properties correlated with composition of colloidal polymers

6. Conclusions and outlook 


\section{Introduction}

The use of well-defined inorganic nanocrystals as reagents for the construction of novel colloidal compounds or assemblies is an emerging approach for the preparation of complex materials with hierarchical structure. A number of reports have described the concept of using inorganic nanoparticles (NPs) as "artificial atoms" for the formation of colloidal crystals and superlattices, as pioneered by Murray and coworkers.[1-3] Shape control of inorganic NPs later extended the structural complexity of these systems by introducing intricate nanocrystalline architectures in the form of nanorod, tetrapod, and dendritic morphologies, as first demonstrated for cadmium chalcogenide NPs.[4-9] Chemical transformations also exist which enabled the formation of "artificial molecules" containing two or more discrete nanoparticle domains within a single nano-object. Examples of these heterostructured nanomaterials include inorganic heterodimer NPs by Xu,[10-11] Teranishi,[12] Sun,[13-14] and Hyeon and coworkers,[15] which have been comprehensively reviewed elsewhere.[16-18] More recently, a "nanoparticle total synthesis" approach was developed for the formation of complex trimers, tetramers, and oligomeric species by sequential nanoparticle formation and bond forming reactions, which expanded the use of preformed nanocrystals as reagents for synthetic transformations .[19-21]

Building on the concept of using nanoparticles as chemical precursors, the use of preformed nanoparticles as "colloidal monomers" has received recent attention for the formation of one-dimensional (1-D) mesostructures, or "colloidal polymers" (also termed "nanopolymers" or "nanochains"). These colloidal monomers form linear assemblies through attractive, and directional, interparticle interactions (e.g., electrostatic interactions, chemical bonds, magnetic interactions). A key challenge in the synthesis of colloidal monomers is in developing synthetic methods to embed anisotropic character to nanoparticle materials to enable strongly associative 
1-D interactions. The formation of colloidal polymers requires that these interparticle associations be strong enough to overcome thermal fluctuations, and that these anisotropic interactions be highly efficient with minimal side reactions to form linear assemblies with reasonable degrees of polymerization (DP).

In contrast to the high degree of structural control afforded from the synthesis of classical molecular polymers, methods to tune fundamental structural features, such as, chain length (DP), composition (copolymers), and architecture (linear, branched, etc.) are still being developed for NP-based colloidal polymer systems. Applying these "principles of polymerization"[22] to the assembly of inorganic nanoparticles is expected to enable access to complex nanocomposite materials that cannot be fabricated via current synthetic methods. However, to our knowledge, the use of inorganic nanoparticles to form colloidal polymers has not been reviewed in terms of monomer types, polymerization mechanisms, or methods for controlling fundamental polymer structural parameters.

Herein, we classify and review the colloidal polymerization of inorganic nanoparticle monomers by applying the conceptual framework provided by polymer science to these mesoscopic systems. The descriptive nomenclature used for classical polymers is used to define more explicitly the types of colloidal polymers that have been prepared in terms of DP, architecture, and composition (for binary or complex NP assemblies). This Review includes descriptions of inorganic nanoparticle types useful for the formation of colloidal polymers with examples chosen to demonstrate control over key structural parameters, and correlation with emergent optical, electrical and electrochemical properties.

\subsection{Types of colloidal polymerizations}


We propose a classification scheme which divides colloidal polymerizations into categories by the interparticle interactions between NP monomers directing the formation of colloidal assemblies (Scheme 1). In classical polymer science, polymers are typically classified by their respective polymerization mechanisms (e.g., step-growth versus chain-growth) which further provides insight into the processes affecting the degree of polymerization (DP), polydispersity $\left(\mathrm{M}_{\mathrm{w}} / \mathrm{M}_{\mathrm{n}}\right)$ and polymer structural features.[22] To date, the vast majority of colloidal polymers are formed via step-growth type processes. Furthermore, the preparation of colloidal polymers has been achieved by harnessing either permanent chemical bonds, or supramolecular associations between NP units.[23-28] However, fundamentally different colloidal monomer types and interparticle binding motifs have been used to form colloidal polymers that deviate from classical polymerization reactions of small molecule monomers. Colloidal polymers are synthesized by harnessing interparticle associations of nanoparticle monomers, and these associations depend greatly on features such as crystal phase, morphology, and ligand functionalization of the colloidal monomers utilized. Hence, the classification of existing colloidal polymers can best be described by the nature of the interparticle associations employed to promote 1-D assembly of nanoparticle monomers (Scheme 1).

\section{INSERT SCHEME 1 AND SCHEME 1 CAPTION}

Interparticle interactions utilized for the formation of colloidal polymers include electrostatic or magnetic dipolar interactions, hydrophobic (non-covalent) interactions of the ligand shells, multifunctional ligands (covalent bonds), direct NP-NP coupling (e.g., metal bonds, ionic bonds), oxidation, and in some cases Ostwald ripening and recrystallization. Interparticle associations directing colloidal polymer formation are divided into three general 
categories based on the origin of the colloidal polymerization, namely, a) ligand-directed; b) surface-directed; or c) dipole-directed associations (Scheme 1).

We describe ligand-directed formation of colloidal polymers as 1-D associations of NPs dictated by the chemical interactions of surface ligands between neighboring particles (Scheme 1a). These ligand-driven associations between NP monomers have been produced by noncovalent interactions (e.g., electrostatic interactions, hydrogen bonding), the formation of covalent bonds, or metal-ligand associations using multi-functional small molecule ligands as colloidal linkers. Electrostatic interactions arise from functional ligands having a net electric charge which directs the formation of linear nanoparticle assemblies (Scheme 1a-1). These interparticle electrostatic interactions of as synthesized nanoparticles are typically isotropic and repulsive in nature due to nanoparticle ligands having like charge (e.g., citrate-capped, AuNPs). However, these interparticle electrostatic interactions can be modulated by judicious choice of reaction conditions ( e.g., by the addition of salts), which has been proposed to form species such as colloidal dimers that promote 1-D nanoparticle assembly.[29-31]

The second subcategory for ligand-directed formation of colloidal polymers deals with multifunctional ligands (also referred to as "molecular linkers" in the literature) or the use of small molecule ligands to form a chemical bond between two otherwise distinct NPs (Scheme 1a-2). The seminal work of Mirkin and coworkers demonstrated the viability of using complementary oligonucleotide thiol ligands on disparate AuNPs to promote NP association into network structures.[32] However, the formation of linear colloidal polymers using multifunctional ligands has the added challenge of imparting directional 1-D NP assembly through surface bound small molecule linkages. For example, linear colloidal polymers based on streptavidin complexes have been synthesized by preferential binding of biotin disulfides to more 
reactive surface sites on the terminal facets of gold nanorods (AuNRs).[33] The inherent shape anisotropy of these nanorods enabled placement of ligands selectively onto terminal facets at two diametrically opposed ends of each colloid, which resulted in the formation of difunctional nanoparticles capable of selective 1-D colloidal polymerization. The chemical selectivity of these nanorod colloidal monomers is possible due to differences in curvature and chemical nature of the surface atoms located on terminal and lateral facets, which results in different binding affinity of ligands for these two surfaces.

The third subcategory for ligand-directed formation of colloidal polymers is through noncovalent interactions of nanoparticles or the use of ligands which preferentially associate and result in nanoparticle agglomeration to form linear assemblies of NPs (Scheme 1a-3). This subcategory includes "patchy particles" which have phase separated mixtures of ligands on the nanoparticle surface and enable NPs to self-assemble through favorable non-covalent interactions of like "patches." An example of this type of system is the self-assembly of AuNRs through the complementary interparticle interactions of zwitterionic glutathione ligands installed at nanorod termini.[34]

Ligand-directed assembly of colloidal polymers is frequently sensitive to $\mathrm{pH}$ and ionic strength of the reaction medium. Compositional changes in the inorganic core can change the efficiency of the directing ligands to bind to the NP surface(s), but otherwise play a minimal role in the ligand-directed formation of colloidal polymers. In addition, crystallographic changes to the NP core can affect the selectivity of ligand binding to NP surfaces, which can be vital for the installation of directional ligand interactions (such as AuNRs).

Surface-directed formation of colloidal polymers (Scheme 1b) occurs through direct contact of inorganic nanoparticle surfaces, and can further be described by oriented attachment, 
or nanowelding approaches. Oriented attachment is the self-assembly of NPs to form larger single crystals by sharing of common crystallographic orientations. Colloidal polymers synthesized by oriented attachment mechanisms can exhibit linear, cyclic, branched, or network architectures (Scheme 1b-1). Oriented attachment of nanoparticles to form colloidal polymers is sensitive to crystallographic changes in the inorganic NP which affect both surface chemistry and effective shape of these colloidal monomers. An elegant example of colloidal polymers formed via oriented attachment is the controlled nucleation, growth, and subsequent oriented attachment of PbSe NPs to form a variety of colloidal polymer architectures.[35] By comparison, nanowelding systems are characterized by the aggregation of NPs to form larger assembled structures (without preferential crystallographic orientation), and have been synthesized by chemically induced destabilization or removal of passivating ligands from the NP surface (Scheme 1b-2). Removal of passivating ligands, or nanowelding, of spherical NPs was observed to uncontrollably aggregate NPs into 3-D networks of irregular size and morphology. However, the preparation of colloidal polymers has been demonstrated using AuNP-tipped CdSe nanorods, where ligand destabilization of AuNP "tips" resulted in the tunable formation of nanorod networks.[36]

Dipole-directed colloidal polymerization (Scheme 1c) arises from through space coupling of charge or spin dipoles inherent to the inorganic NP cores of colloidal monomers. These dipolar interparticle interactions can be mitigated by ligand-induced repulsive interactions or increased temperature. Additionally, the linearity of self-assembled structures from dipolar NPs is strongly dependent on the balance between dipolar interactions and van der Waals interactions between the constituent particles. Electrostatic dipoles arise from anisotropic crystal structures within NPs, and these dipoles must be strong enough to overcome both thermal fluctuations and 
ligand-induced repulsive interactions to form colloidal polymers (Scheme 1c-1). The seminal work of Kotov and coworkers utilized CdTe colloidal monomers, which have been extensively studied.[37] Ligand-induced repulsive interactions are minimized for CdTe NPs by removal of excess ligands from NP dispersions prior to colloidal polymer formation.

Conversely, the formation of colloidal polymers has been demonstrated for coupling of NPs possessing magnetic (spin) dipoles from dipolar associations of either ferromagnetic, or ferrimagnetic colloidal monomers (Scheme 1c-2).[38-50] For example, the seminal work of Thomas and Hess and coworkers described the formation of dipolar colloidal polymers via magnetic associations of ferromagnetic polymer coated cobalt nanoparticles (CoNPs).[51-52] Ferrimagnetic colloidal polymers have been observed in biological systems, most notably in the formation of magnetosome chains composed of $\mathrm{Fe}_{3} \mathrm{O}_{4}$ NPs formed in magnetotactic bacteria.[53]

Each of the above colloidal polymer classifications (from Scheme 1) will be applied to various systems to discuss key polymer parameters such as DP, composition (copolymers), and architecture. We will also discuss the mechanisms governing the assembly of colloidal monomers for the formation of complex colloidal polymer architectures and compositions. The reader is referred to other reviews for general NP assembly,[54-58] the use of templates,[59-72] metallization of (bio)organic polymers,[73-74] polypeptide-grafted particles, [75] field-directed assembly,[76-77] assembly at liquid-liquid interfaces,[78-80] microribbons,[81-82] micron-sized rods, [83-84] or other forms of self-assembly where associative interactions of inorganic colloids play a lesser role in directing the colloidal polymer structure.

\subsection{Synthesis of colloidal monomers}

Modern advances in the synthesis of inorganic nanoparticles provide the basis for the development of colloidal monomer systems (Scheme 1) to embed the shape anisotropy and 
differential functionalization necessary to promote 1-D colloidal polymerization versus 2-D or 3D assembly (Fig. 1a). In general, synthetic methods have been developed to control the size of nanoparticles based upon metals and semiconductors. The use of polymeric ligands and polymeric assemblies have also been reported to afford a wide range of well-defined inorganic nanoparticles.[43, 85-92] Furthermore, in the case of certain systems, methods to control NP architecture have been demonstrated to prepare 1-D nanorods or more complex branched materials (e.g., tetrapods).[5-7, 93-94] Notable and extensively studied examples include Ag and Au nanorod materials (Fig. 1b),[95-105] along with II-VI semiconductor tetrapods and dendritic nanocrystals (Fig. 1c).[8-9, 16-18, 93, 106] Methods to control NP size and composition have been extended to enable the synthesis of materials having multiple NP domains, as demonstrated by the seminal work on heterodimeric NPs (i.e., Janus particles, Fig. 1d),[10-18, 92, 107] and the differential binding affinity of ligands for terminal nanorod facets has been utilized for the selective placement of assembly-directing ligands to create difunctional colloidal monomers.[108-111] Similar heterostructured nanocrystals have been prepared using metalsemiconductor nanocomposites based on II-VI semiconductor nanorods capped with metal NPs selectively bound to the nanorod termini (i.e., NP "tips").[112-114] In these metalsemiconductor NRs, the wurtzite structure of the semiconductor NR promoted deposition of metallic NPs to the NR termini, which created colloidal surfaces that were differentially functionalized with multi-functional ligands that promoted 1-D colloidal polymerization (Fig. 1e).[112-114] The compositional anisotropy afforded by tipped nanorod heterostructures has resulted in a variety of colloidal polymer forming strategies through selective reactivity of NP tips, and colloidal polymers based on tipped nanorods are present in all of the major categories 
described in this Review. Conversely, highly branched colloidal polymers were obtained through colloidal polymerization of metal NP tips on more complex tetrapod architectures (Fig. 1f).[36]

\section{INSERT FIGURE 1 AND FIGURE 1 CAPTION}

\section{Ligand-directed formation of colloidal polymers}

For classification purposes, ligand-directed colloidal polymerization is a broad category which includes interactions arising from surface bound organic ligands on individual NPs resulting in the 1-D (or lightly branched) self-assembly of inorganic NPs (Scheme 1a). These interparticle ligand interactions have promoted colloidal polymerization of NPs using electrostatic interactions, multifunctional ligands, or non-covalent interactions, as discussed in separate sections below. AuNPs have been the most widely investigated colloidal monomer for ligand-directed formation of colloidal polymers in the literature due to both facile control of NP size and surface chemistry via functionalization with thiol-containing ligands.[115] In most cases, the inorganic core does not intrinsically possess sufficient shape anisotropy to enable spontaneous 1-D assembly under practical synthetic conditions. However, functionalization of nanoparticle surfaces with small molecule ligands based on terminal thiols, or block copolymer ligands have been investigated to form "patchy" asymmetrically functionalized colloids from otherwise isotropic particles. Often, multiple mechanisms for colloidal polymerization operate simultaneously and only the dominant pathway directing linear assembly is of interest for a given report. However, in some cases, competitive forces are intentionally utilized in the formation of colloidal polymers to control key polymer parameters, as discussed below in the section for colloidal polymers directed by ligand-induced electrostatic charge. 


\subsection{Ligand-induced electrostatic charge}

As previously discussed, linear colloidal polymers from ligand-induced electrostatic charge have primarily been investigated for functional AuNP systems. A typical synthesis of spherical AuNPs uses citrate ligands, and affords negatively charged AuNPs in aqueous media.[116-118] One of the key questions regarding the formation of colloidal polymers from these noble metal NPs is the driving force for the formation of linear assemblies from isotropic AuNPs, which would be expected to form 3-D network colloidal polymers.[119] A number of reports have investigated AuNP colloidal polymerization and the formation of complex structures via two general mechanisms: (1) phase separation of binary mixtures of surface bound ligands to generate charge anisotropy (electrostatic dipole), or 2) the asymmetric structure of NP dimers imparting charge anisotropy, as opposed to individual colloidal monomers. Mechanistic discussions for a given system are further complicated by possible contributions from thiolterminated ligands which have been proposed to result in the formation of spin dipoles on small $(\mathrm{D}<2 \mathrm{~nm})$ Au clusters.[120]

One of the early proposed mechanisms for linear assembly of AuNPs postulated the spontaneous phase separation of binary mixtures of charged and neutral surface bound ligands on NP surfaces to generate a net charge dipole that promoted 1-D polymerization. Mann and coworkers investigated the mechanism for the self-assembly of AuNPs $(\mathrm{D}=13 \mathrm{~nm})$ functionalized with a mixture of citrate and mercaptoethanol ligands, and the formation of statistical copolymers with branched architectures was achieved by incorporation of larger AuNPs $(D=20 \mathrm{~nm})$.[121-122] Negatively charged, citrate capped, AuNPs $(\mathrm{D}=13 \mathrm{~nm})$ were synthesized using the citrate reduction of $\mathrm{HAuCl}_{4},[116-118]$ which afforded aqueous NP dispersions. Linear and reticulated assemblies of AuNPs were observed upon addition of 2- 
mercaptoethanol (MEA) to an aqueous dispersion of these AuNPs. The formation of these NP assemblies was attributed to the presence of a mixture of both citrate and MEA ligands, which the authors proposed to promote the formation of an electrostatic dipole via phase separation of ligands on the NP surface.[121] TEM studies of a sample prepared at a molar ratio of MEA to AuNP of 3000:1 revealed looped chains ranging $1-5 \mu \mathrm{m}$ in length, which corresponded to $80-$ 380 AuNPs per colloidal polymer chain $(\mathrm{DP}=80-380)$.

Later investigations by Mann and coworkers on AuNP assembly using this mixed ligand system found the final colloidal polymer architecture to also be dependent on temperature and AuNP diameter (Fig. 2).[122] Binary mixtures with AuNPs having larger diameters (20 nm versus $13 \mathrm{~nm}$ ) resulted in phase separated mixtures at equimolar ratios of AuNPs. However, statistical copolymers were obtained by decreasing the feed ratio of $20 \mathrm{~nm}$ AuNPs (ca. 99:1 small:large AuNPs), where the larger AuNPs served as both branching points and chain stoppers in these assemblies (Fig. 2).[122] The diminished reactivity of larger AuNPs was attributed to the decreased (electrostatic) dipole moment for larger AuNPs, which resulted in termination of colloidal polymer chains (chain stopping agents). These branched colloidal copolymers formed from a large excess of $13 \mathrm{~nm}$ AuNPs were reported to span up to $1300 \mathrm{~nm}$ in length, which corresponded to a DP of ca. 100 NPs. The mechanism for linear assembly of AuNPs through dipolar electrostatic interactions of ligand patches has been utilized by others to explain observations of NP assembly.[123]

INSERT FIGURE 2 AND FIGURE 2 CAPTION 
An alternative proposed mechanism leading to linear assembly of negatively charged AuNPs is based on the anisotropic shape and reactivity of AuNP dimers (Fig. 3).[29] These dimers arise from random collisions of isotropic AuNPs, and the addition of a third AuNP to a dimer (to form a trimer) is favored at the ends of these structures. This "dimer/multimer" mechanism was evaluated theoretically by Kotov and coworkers (for CdTe NPs)[30] and experimentally by Wang and coworkers (for charged AuNPs).[31] The presence of an electrostatic dipole was proposed to contribute to the formation of linear assemblies, in both of these cases.[30-31] However, the formation of linear chains of surfactant stabilized, charge neutral AuNPs (e.g. Au-S-R) was also modeled by using pair potential calculations of potential energy surfaces and Brownian dynamics simulations, where electrostatic dipoles were not required for the formation of 1-D NP chains from isotropically stabilized particles (Fig. 3).[29]

\section{INSERT FIGURE 3 AND FIGURE 3 CAPTION}

Wang and coworkers evaluated assembly behavior of negatively charged, thioglycolic acid capped AuNPs $(\mathrm{D}=14 \mathrm{~nm})$ versus ionic strength or dielectric of the media to develop a theoretical framework in terms of electrostatic repulsion, van der Waals interactions, and dipolar interactions induced by stabilizing ligands.[31] Linear nanoparticle assembly was selectively achieved over 2-D or 3-D agglomeration by controlling the ionic strength (by addition of $\mathrm{NaCl}$ ) or dielectric constant (by addition acetonitrile) of aqueous NP dispersions, where colloidal polymer chain length increased with increasing ionic strength (or decreasing dielectric constant) of the media. Electrostatic repulsive interactions were decreased in the presence of salts (or acetonitrile), which increased the number of successful NP collisions (and colloidal polymer DP) 
due to screening of repulsive electrostatic interactions. However, calculations showed that electrostatic repulsive interactions were stronger than van der Waals interactions, which should have precluded chain formation.

Wang and coworkers further proposed that the electrostatic double layer surrounding associated NPs rearranged into a uniform layer surrounding the entire colloidal chain,[31] which eliminated repulsive interactions between NPs in dimers. These AuNP dimers were found to have asymmetric electrostatic repulsive potentials, where a particle approaching from the side of a dimer would experience stronger electrostatic repulsion than one approaching from the end of the dimer.[31] This mechanistic picture was similar to that previously discussed for neutral, surfactant stabilized AuNPs leading to linear assemblies.[29] However, dipolar interactions were also proposed to contribute to the assembly of these thioglycolic acid capped AuNPs,[31, 124] by citing previous findings that $\mathrm{Au}-\mathrm{S}$ bonds can result in the formation of magnetic dipoles for smaller (1.4 nm) Au clusters.[120] The authors did not distinguish for this system whether thiolterminated ligands contributed to the formation of an electrostatic dipole (due to phase separation of ligands, as per Mann and coworkers[122]) or a magnetic dipole (as observed for thiolpassivated AuNPs having significantly smaller diameters[120]). Nevertheless, a mathematical relationship was derived between the average number of nanoparticles in a chain (DP) and the surface charge density of the particles for linear chains up to approximately $400 \mathrm{~nm}(\mathrm{DP} \approx 30$ NPs).[31]

Recently, several methods were developed to control DP of linear AuNP chains by adjusting the ionic strength of AuNP dispersions.[31, 125-126] In these studies, increased salt concentrations were utilized to screen repulsive interparticle electrostatic interactions and promoted increased agglomeration (assembly) of AuNPs. This approach to NP assembly is based 
on earlier work using cationic species to induce the formation of linear and reticulated chains of AuNPs,[127-128] where the mechanism for the formation of linear assemblies in these systems were in general agreement with the findings of Kotov and Wang and coworkers[29-31, 124] Chen and coworkers studied the effect of ionic strength on the linear assembly of 2naphthalenethiolate capped AuNPs $(\mathrm{D}=15 \mathrm{~nm})$ using a block copolymer ligand encapsulation methodology to enable TEM imaging of the assemblies in the dispersed state, and demonstrated the synthesis of colloidal block copolymers from binary mixtures with smaller (10 nm) AuNPs (Fig. 4).[125] AuNPs in this system were synthesized via the standard citrate reduction of $\mathrm{HAuCl}_{4}$, followed by ligand exchange with 2-naphthalenethiolate. Excess citrate salts were removed during purification by centrifugation prior to studies on NP assembly carried out in DMF, and these AuNPs retained colloidal stability after both purification and ligand exchange of the citrate-capped AuNPs with 2-naphthalenethiolate. Agglomeration of NP dispersions was induced by increasing the ionic strength of the media (with $\mathrm{NaCl}$ or $\mathrm{NaNO}_{3}$ ) to screen electrostatic repulsive interactions between negatively charged NPs. Increasing salt concentrations led to longer NP chains, as observed by TEM imaging, and linear colloidal polymers up to 40 NPs in length were observed at the highest salt concentration studied (0.99 $\mathrm{mM} \mathrm{NaCl}$ ). The authors proposed that the linear aggregation was kinetically controlled and that charge repulsion among AuNPs was the key factor directing linear aggregation, similar to the dimer/multimer mechanisms of Kotov and Wang and coworkers[29-31]

The AuNP assemblies of Chen and coworkers were preserved by in situ encapsulation in frozen block copolymer ligand shells for TEM studies to exclude any drying effects on colloidal polymer structure $\left(\mathrm{PS}_{154}-b-\mathrm{PAA}_{60}, \mathrm{PS}-b-\mathrm{PAA}, \mathrm{M}_{\mathrm{n}}=16,000 \mathrm{~g} / \mathrm{mol}\right.$ for the polystyrene block and $M_{n}=4300 \mathrm{~g} / \mathrm{mol}$ for the poly(acrylic acid) block, $M_{w} / M_{n}=1.15$, Fig. 4).[125] PS- $b$-PAA formed 
micelles under the conditions used for polymer encapsulation $\left(\mathrm{DMF} / \mathrm{H}_{2} \mathrm{O}\right)$, and high temperature destabilization of these polymer mesostructures $\left(\mathrm{T}=110^{\circ} \mathrm{C}\right)$ resulted in AuNPs uniformly coated with PS- $b$-PAA. UV-vis spectra of these assemblies were unchanged before and after polymer encapsulation,[125] which demonstrated that the structures of these assemblies were not altered by this polymer encapsulation methodology.[129] [125]

\section{INSERT FIGURE 4 AND FIGURE 4 CAPTION}

In these PS- $b$-PAA/2-naphthalenethiolate capped AuNPs, preferential end-to-end attachment of AuNPs was found to be strongly dependent on particle diameter for AuNPs having diameters of $5 \mathrm{~nm}, 10 \mathrm{~nm}$, and $15 \mathrm{~nm}$ (Fig. 4).[125] Similar to the dimer/multimer-induced asymmetric electrostatic repulsion mechanism of Wang and coworkers, [29-31] the electrostatic repulsive force experienced by a AuNP having a smaller diameter $(D=5 \mathrm{~nm})$ approaching a chain of AuNPs having larger diameters $(\mathrm{D}=15 \mathrm{~nm})$ was less than that for a AuNP having a more closely matched diameter $(D=10 \mathrm{~nm})$ approaching the same NP chain $(D=15 \mathrm{~nm}$, Fig. 4a). These differences in repulsive forces were attributed to increased electrostatic repulsions between larger NPs (for a given surface charge density),[125] as opposed to reorganization of ligands to form an electrostatic dipole. These mechanistic findings were then utilized to form oligomeric colloidal block copolymers having segments enriched in AuNPs of differing diameters (AuNP diameters $=10 \mathrm{~nm}$ and $15 \mathrm{~nm}$; Fig. 4b). End-functional "tadpole" like structures consisting of chains of $15 \mathrm{~nm}$ AuNPs attached to $60 \mathrm{~nm}$ AuNPs were also synthesized by control over the ionic strength of the media where AuNP-AuNP interactions were found to be most strongly repulsive between AuNPs having larger diameters (Fig 4c-i). The range of 
accessible chain lengths from these colloidal polymerizations were not discussed for either of these copolymer compositions, although TEM images suggest that these assemblies consisted of approximately 20 NPs per chain.[125]

Linear colloidal polymers capable of reversible assembly were synthesized by Yin and coworkers in their investigation of the role of salt in the self-assembly of AuNPs $(D=10 \mathrm{~nm})$ in ethanol, which demonstrated that thiol-terminated ligands were not required for linear assembly of AuNPs.[126] AuNPs were synthesized by the standard citrate reduction of $\mathrm{HAuCl}_{4}$ and subsequently functionalized with bis(p-sulfonatophenyl)phenylphosphine dihydrate dipotassium salt (BSPP) after ligand exchange to improve colloidal stability. The main purpose of this study was to clarify earlier reports in the literature that aggregation of negatively charged AuNPs was induced by the addition of ethanol.[130-131]

This study on the aggregation of AuNPs by Yin and coworkers determined that the key factor affecting AuNP aggregation in ethanol or ethanol/water mixtures was residual salt, as opposed to ethanol itself, for both citrate and BSPP ligand capped AuNPs.[126] Increased aggregation rates were associated with increased salt concentrations in wet ethanol, which the authors noted were introduced into the system after purification of the (salt containing) synthetic reaction mixture. Colloidal polymer chain length increased with increasing salt concentration in ethanol (determined by UV-vis), although DP was not explicitly discussed in this report. The reversible polymerization of these AuNP colloidal polymers in ethanol/water mixtures was demonstrated by dissociation of AuNP chains upon dilution of the system with water or evaporation of ethanol, but only when the NPs were passivated with a sufficiently tightly binding ligand such as BSPP. This study supported the mechanisms of Wang and coworkers, [29, 31] and 
demonstrated the need for precise salt concentrations and nanoparticle purity when targeting the linear assembly from ligand-directed colloidal polymerization.

Xia and coworkers utilized tunable electrostatic repulsive interactions to control DP and monomer spacing in their study of in situ encapsulation of AuNP colloidal polymers.[132] AuNPs $(\mathrm{D}=17.7 \mathrm{~nm})$ were prepared by reduction of $\mathrm{HAuCl}_{4}$ with sodium citrate, and the formation of linear and branched assemblies was triggered by the addition of mercaptoacids with varying alkyl segments $\left(\mathrm{HS}\left(\mathrm{CH}_{2}\right)_{\mathrm{n}} \mathrm{COOH}, \mathrm{n}=2,10,15\right)$ to AuNP dispersions in wet ethanol (98:2 ethanol:water). The mercaptoacids were proposed to completely replace the citrate ligands present on AuNPs, and remained negatively charged under these conditions. The large excess (> $0.01 \mathrm{M}$ ) of these surfactants reduced interparticle electrostatic repulsive interactions between AuNPs by screening NP charges due to the increased concentration of charged species. The observed assembly behavior was in agreement with the predicted decrease in AuNP Debye length with increasing surfactant concentration,[132] based on previous reports of Bibette and Xia and coworkers[133-134]

AuNP spacing in these colloidal polymers was correlated with increasing alkyl chain length of the surfactant, as confirmed by tracking of the localized surface plasmon resonance (LSPR) of colloidal monomers by UV-vis spectroscopy.[135-136] Additionally, ligand size affected the kinetics of colloidal polymer formation, where assembly times ranged from days to weeks with increasing mercaptoacid alkyl chain length. Conversely, aggregation rate and colloidal polymer chain length (DP) were observed to increase with increasing surfactant concentration. The number of AuNPs per linear chain was controlled for oligomeric colloidal polymers consisting of up to ca. 20 NPs per chain upon incubation in $1 \mathrm{mM} 3$-mercaptopropionic acid solution for 24 hours. Larger, reticulated (network) NP assemblies were observed at higher 
surfactant concentration and longer reaction time. Colloidal polymer chains were then fixed in either silica (by the addition of tetraethyl orthosilicate), or in gelatin (by a microfluidic approach). Nanoparticle spacing and colloidal polymer architecture were minimally affected by these encapsulation approaches, as confirmed by UV-vis spectroscopy and SEM imaging, respectively.

Kumacheva and coworkers utilized both hydrophobic and electrostatic interactions to form linear and globular colloidal polymers from charged AuNPs $(D=23 \mathrm{~nm})$ capped with a mixture of cetyltrimethylammonium bromide (CTAB) and polystyrene ligands.[137] AuNPs capped with CTAB ligands were subjected to ligand exchange with thiol-terminated polystyrene $\left(\mathrm{M}_{\mathrm{n}}=5,000\right.$ to $\left.50,000 \mathrm{~g} / \mathrm{mol}\right)$ to afford colloidal monomers coated with a mixture of polystyrene and charged CTAB ligands. NP assembly was systematically controlled by tuning solvent quality for the polymer ligands and screening of repulsive electrostatic interactions afforded by retained $\mathrm{CTAB}$ ligands. These electrostatic associations were found to be strongly dependent on ionic strength, concentration of water in DMF/water mixtures, and on the amount of THF added to these colloidal dispersions .

In this AuNP system capped with mixed ligands of PS and CTAB, colloidal polymer conformational transitions (chain versus globule) were governed by the competition of attractive hydrophobic forces from PS ligands and repulsive electrostatic forces from the charged AuNPs.[137] A phase diagram for polystyrene ligands of various molar masses versus concentration of water was determined, ranging from isolated AuNPs (all PS $\mathrm{M}_{\mathrm{n}}$ studied, low concentration of water), NP globules (high PS $\mathrm{M}_{\mathrm{n}}$, moderate concentration of water), and NP chains (all PS $\mathrm{M}_{\mathrm{n}}$ studied, high concentration of water). Linear oligomeric AuNP chains having the longest lengths in this report ( $\mathrm{DP} \approx 25 \mathrm{NP}$ based on available TEM images) were obtained 
from AuNPs capped with PS ligands of the lowest molar mass $\left(M_{n}=5,000 \mathrm{~g} / \mathrm{mol}\right)$ at relatively high volume fraction of water in DMF (15\% water v/v). Polymer ligand molecular weight was also demonstrated to impact interparticle spacing between colloidal monomers. Solution light scattering was utilized in conjunction with TEM studies to observe structural transitions between linear chains and globular assemblies of these polystyrene coated spherical AuNPs, which provided additional evidence for the imaged structures representing the dispersed state.[137]

Wang and coworkers recently utilized the electrostatic repulsion mechanism for AuNP chain formation to synthesize oligomeric colloidal block copolymers consisting of segments enriched in both small $(\mathrm{D}=25 \mathrm{~nm})$ and large $(\mathrm{D}=36 \mathrm{~nm})$ AuNPs capped with $\mathrm{pH}$ sensitive polymer ligands (poly([2-(dimethylamino)ethyl] methacrylate), Fig. 5).[138] Spherical AuNPs were synthesized by $\mathrm{Ag}(\mathrm{I})$-assisted reduction of $\mathrm{HAuCl}_{4}$ with citrate in boiling water,[139] and citrate capped AuNPs were then subjected to ligand exchange with (poly([2(dimethylamino)ethyl] methacrylate) complexed with disodium citrate to prepare the polymer coated NPs used for aqueous self-assembly. Colloidal polymer chain length increased with decreasing $\mathrm{pH}$ of the reaction medium due to protonation of complexed citrate ions which resulted in diminished interparticle electrostatic repulsive interactions (increased zeta potential).

Linear assembly in these systems was attributed to the dimer/multimer-induced asymmetric electrostatic repulsion mechanism of Wang and coworkers, ${ }^{98,100-101}$ and a minimal dependence of colloidal polymer chain length versus the diameter of NP colloidal monomers was observed (Fig. 5).[138] However, similar colloidal polymer chain lengths resulted in a decreased number of NPs per chain (DP) for increasing AuNP diameter (up to $\mathrm{D}=36 \mathrm{~nm}$ ). The largest linear chains in that report were oligomeric with lengths of approximately $300 \mathrm{~nm}$ (based on TEM images), which corresponded to DPs ranging from 5 - 15 NPs, depending on NP diameter. 
AuNP diameter also contributed to the electrostatic repulsion of individual chains such that endto-end coupling of chains consisting of AuNPs of differing diameters was disfavored for binary mixtures of small and large AuNPs. Oligomeric colloidal block copolymers were obtained by blending chains of $25 \mathrm{~nm}$ AuNPs $(3 \pm 1$ NPs per chain) and chains of $36 \mathrm{~nm} \mathrm{AuNPs}(2 \pm 1$ NPs per chain) in water, followed by stepwise adjustment from pH 3.0 to 2.5 (Fig. 5). This system, in particular, illustrated the ability to control colloidal polymer composition using variations of $\mathrm{pH}$ to induce ligand-directed electrostatic assembly of AuNPs of differing diameters.

\section{INSERT FIGURE 5 AND FIGURE 5 CAPTION}

\subsection{Multifunctional ligands}

For the purposes of forming colloidal polymers, the term "multifunctional ligand" refers to small molecules which induce the formation of linear (or lightly branched) NP assemblies through chemical associations (or bonds) between ligands on disparate colloidal monomers. Colloidal polymers based on multiple associations of ligands with NPs have been primarily investigated using AuNPs, which can be attributed to the extensive studies of Au surface functionalization using thiols and disulfides.[140] However, isotropic functionalization of spherical particles with ligands that promote association of NPs typically affords highly branched, or network, colloidal polymers. Therefore, synthetic routes to colloidal polymers from multifunctional ligands require methods for either anisotropic functionalization of NPs, or kinetically controlled conditions to suppress 3-D colloidal polymer network formation.

The assembly of AuNPs with oligonucleotides and other biomolecules has been widely utilized for sensing applications based on the early work of Mirkin and coworkers,[141] which 
has been extensively reviewed elsewhere.[142-144] However, the formation of linear nanoparticle assemblies via these methods have generally relied on preformed DNA templates for NP associations.[145] Hence, the synthesis of colloidal polymers of controllable architecture and composition requires precise functionalization and assembly methods of NP colloidal monomers.

One related technique which was utilized for the formation of alternating colloidal copolymers was based on the polymerase chain reaction (PCR) for replication of chiral doublestranded DNA on AuNPs (Fig. 6).[146-150] In this system, AuNPs of two distinct diameters (10 $\mathrm{nm}$ and $25 \mathrm{~nm}$ ) were functionalized with thiolated reverse and forward primers, utilizing stoichiometric control to limit the number of primers per AuNP. Repetitive PCR cycles with small and large AuNPs resulted in oligomeric alternating plasmonic copolymers consisting of up to approximately 12 NPs per colloidal polymer chain as characterized by TEM (Fig. 6a).[146] The number of PCR cycles used to synthesize colloidal polymers correlated with increasing chain length or degree of polymerization (Fig. 6b-i). Cycle number also correlated with the circular dichroism spectra for these plasmonic colloidal polymers, which afforded tunable chirality of these structures. While other examples exist which demonstrate preferential alternation of colloidal monomers within linear assemblies, this PCR methodology is the only example to date which provides a synthetic route to truly alternating colloidal copolymers. Related work with AuNRs also afforded a means for enhancing the specificity of DNA oligomers.[151-152]

INSERT FIGURE 6 AND FIGURE 6 CAPTION 
Stellacci and coworkers demonstrated the selective functionalization of NPs with multifunctional ligands by installation of 11-mercaptoundecanoic acid (MUA) activated by Nhydroxysuccinimide at two diametrically opposed points, or "point defects" on AuNPs $(\mathrm{D}=10$ nm) having stripe-like ligand domains (Fig. 7).[153] Hydrophobic AuNPs were synthesized using a modified Schiffrin method,[154-157] which afforded AuNPs capped with a binary mixture of 1-nonanethiol and 4-methylbenzenethiol. This binary ligand mixture was reported to form stripe-like domains of ligands on the NP surface, as previously reported for nanoparticulate Au surfaces having high curvature.[158-160] Ligands at the poles of these spherical AuNPs were preferentially exchanged with thiol-terminated carboxylic acids (i.e., MUA).[153] This selective functionalization of "point defects" with carboxylic acid functionality afforded $\mathrm{A}_{2}$ colloidal monomers (i.e., $\mathrm{A}_{2}$ and $\mathrm{B}_{2}$ refer to difunctional monomers used for step-growth polymerization[22]) which could then be polymerized via interfacial polymerization methods by addition of $\mathrm{a}_{2}$ small molecule monomer (1,6-diaminohexane). This interfacial polymerization approach afforded colloidal polymer films consisting of linear nanoparticle assemblies (DP = 3 20 NPs per chain), as observed by TEM (Fig. 7). Interparticle distances were correlated with variation of linker length in the small molecule used for the formation of these colloidal polymers (e.g., O,O'-bis(2-aminoethyl)octadecaethylene glycol (PEG-diamine, n=20)). Blends of difunctional AuNPs having differing diameters $(10 \mathrm{~nm}$ and $20 \mathrm{~nm})$ were reported (in supporting information). Similar studies by Stellacci and coworkers were conducted using superparamagnetic iron oxide NPs, where an increase in blocking temperature for colloidal polymers in comparison to individual iron oxide NPs was observed .[161]

\section{INSERT FIGURE 7 AND FIGURE 7 CAPTION}


The synthesis and self-assembly of AuNRs to form colloidal polymeric materials have been extensively investigated.[97, 108] The inherent shape anisotropy of AuNRs, in conjunction with the differential binding of ligands to the terminal or lateral facets, can be utilized to promote 1-D assembly of NPs into colloidal polymers. In particular, the ability to selectively install ligands at nanorod termini affords the opportunity to synthesize $A_{2}$ monomers[22] capable of 1D assembly. For example, the selective ligand placement of biotin disulfides at AuNR termini enabled controlled formation of colloidal polymers using biotin-streptavidin multivalent ligands, as first demonstrated by Murphy and coworkers[33] Cetyltrimethylammonium bromide (CTAB) ligands used during the synthesis of AuNRs were postulated to bind preferentially to lateral NR facets, which left the terminal facets of the nanorods more exposed for further functionalization with biotin disulfides. Treating dispersed nanorod-biotin conjugates with streptavidin thus resulted in linear colloidal polymers having (AuNR-biotin-avidin-biotin) n $_{\mathrm{n}}$ connectivity.[33] Although the degrees of polymerization were not discussed for these colloidal polymers, the formation of oligomeric colloidal polymers having at least 10 AuNRs were obtained as indicated by TEM images.

Thomas and coworkers extensively investigated the formation of linear assemblies of AuNRs using a variety of approaches based on multifunctional ligands (Fig. 8).[162-165] In these systems, the selective functionalization of terminal nanorod facets with $\alpha, \omega$-alkanedithiols of varying hydrocarbon length ( $3-9$ carbons) was a crucial factor in obtaining difunctional colloidal monomers capable of 1-D assembly. In one study,[162] CTAB coated AuNRs (aspect ratios 2.2 and $2.9, \mathrm{~L} \approx 50 \mathrm{~nm}$ ) were treated with multifunctional ligands to trigger linear nanoparticle assembly in acetonitrile/water (4:1 v/v) affording chemically bonded AuNR 
colloidal polymers having up to 20 NRs per chain (Fig. 8c). Colloidal polymerization of these difunctional nanorods was preceded by an incubation period, during which AuNRs were functionalized with the $\alpha, \omega$-alkanedithiol ligands, and this incubation period increased with increasing alkyl chain length in the multifunctional ligand.[162] Upon reaching a critical concentration of difunctional AuNRs (colloidal monomers), second order kinetics were observed for colloidal polymer formation. These kinetic observations were consistent with the stepwise mechanism proposed for polymerization of these difunctional AuNRs (Fig. 8a,b). The energies of activation for dimerization of nanorods were estimated from Arrhenius plots to be 85.9, 80.2, and $33.8 \mathrm{~kJ} / \mathrm{mol}$, for AuNRs possessing $\mathrm{C}_{9}, \mathrm{C}_{8}$, and $\mathrm{C}_{5}$ multifunctional ligands, respectively. Dimerization was more highly favored in the case of nanorods possessing shorter dithiol linkers, which was attributed to activation controlled processes (rather than diffusion controlled) with relatively large activation energies for these polymerizations. This kinetic investigation was perhaps the first correlation of a stepwise kinetic model with the formation of a colloidal polymer.[162]

\section{INSERT FIGURE 8 AND FIGURE 8 CAPTION}

The seminal report by Banin and coworkers demonstrated the synthesis, functionalization, and colloidal polymerization of CdSe nanorods $\left(\mathrm{L}_{\text {nanorod }}=20-30 \mathrm{~nm}, \mathrm{D}_{\text {nanorod }}\right.$ $=3-4 \mathrm{~nm}$ ) functionalized with AuNP-tips $\left(\mathrm{D}_{\text {AuNP-tip }}=2-4 \mathrm{~nm}\right)$.[112] An attractive feature of these metal-tipped nanorod systems for colloidal polymerization is the ability to differentially functionalize metallic tips by selective installation of $\alpha, \omega$-dithiol molecules to promote metal coordination with other AuNP tips.[112] These tipped nanorods have been utilized for the 
formation of linear and star-like colloidal polymer architectures through selective functionalization of AuNP tips with multifunctional ligands.[112-114] Using this approach, colloidal polymers having approximately 15 NPs per chain (as observed by TEM) were reported. [112]

More complex branched colloidal polymer architectures were later obtained for the same

tipped nanorod system using avidin-biotin interactions, where AuNP tips on CdSe nanorods were functionalized with biotin disulfide and/or avidin to form " $\mathrm{A}$ ', $\mathrm{A}_{2}$, and $\mathrm{B}_{3}$ type"[22] colloidal monomers (Fig. 9a).[114] Colloidal polymer architecture was controlled by variation of the feed ratios of these nanorods and small molecule ligands to prepare AuNRs carrying: (1) a single biotin functionality (A' colloidal monomer), (2) a single avidin functionality with three remaining sites for biotin-nanorod conjugation ( $\mathrm{B}_{3}$ colloidal monomer), or (3) two biotin functionalities per nanorod ( $\mathrm{A}_{2}$ colloidal monomer, Fig. 9a). Subsequent exposure of A' colloidal monomers to avidin ( $\mathrm{B}_{4}$ functionality ) in a 2:1 molar ratio resulted in a mixture of dimers and star-like colloidal polymer architectures consisting of up to 10 NRs, based on TEM studies (Fig. $9 b, d)$. By comparison, linear trimers were preferentially formed by blending $A_{2}$ colloidal monomers with $\mathrm{B}_{3}$ colloidal monomers in a 1:2 ratio (Fig. 9c).[114]

\section{INSERT FIGURE 9 AND FIGURE 9 CAPTION}

\subsection{Non-covalent interactions}

For the purposes of forming colloidal polymers, the term "non-covalent interactions" refers to linear (or lightly branched) assemblies arising from attractive interactions of surface bound ligands between NPs, which do not result in the formation of covalent bonds. One of the major challenges with these systems is the selective functionalization of regions of colloid 
surfaces to promote 1-D interparticle associations. For example, binary mixtures of ligands have been used to create phase separated ligand domains on NPs to form "patchy particles" which further undergo 1-D self-assembly to maximize energetically favorable interactions of NP ligand shells.[166-167] Hydrophobic ligand patches on nanorod termini have been utilized to favor assembly of like NP segments in polar media due to the hydrophobic effect.[168-169] Hydrophobic interactions have also been utilized to incorporate isotropically functionalized NPs into cylindrical block copolymer micelles for 1-D assembly.[170-176] Alternatively, complementary zwitterionic interactions of ligands at nanorod termini have also been utilized to generate 1-D assemblies.[34]

Block copolymer micelles have been utilized to direct the linear assembly of inorganic colloids, as was recently reviewed.[177] Early work by Taton and coworkers demonstrated that sphere to string transitions in block copolymer micelles could be utilized to direct the assembly of AuNPs.[178-179] Dodecanethiol capped AuNPs ( $\mathrm{D}=12,32$, and $52 \mathrm{~nm}$ ) were encapsulated in PS- $b$-PAA block copolymer ligands to afford water dispersible AuNPs. Colloidal polymerization of AuNPs was then triggered by adjusting $\mathrm{pH}$ or ionic strength of the media to afford linear AuNP assemblies containing up to approximately 40 NPs per chain (based on available TEM images) due to a sphere to string transition of the block copolymer ligand shell.[178-179]

Chen and coworkers have also demonstrated a variety of colloidal polymer motifs based on controlled destabilization of polymer ligands and micelle encapsulated AuNPs (Fig. 10).[170$175,180]$ While the vast majority of colloidal polymers follow step-growth kinetics, Chen and coworkers observed an unconventional chain growth mode for the linear assembly of AuNPs (D $=16 \mathrm{~nm}$ ) encapsulated in polystyrene-block-poly(acrylic acid) (AuNP@PS- $b$-PAA).[180] The 
characteristic feature of this chain growth type mechanism was the formation of long chain colloidal polymers $(\mathrm{DP} \approx 200 \mathrm{NPs}$ ) at low colloidal monomer conversions (Fig. 10c). Citrate capped AuNPs functionalized with 1,2-dipalmitoyl-sn-glycero-3-phosphothioethanol were encapsulated in block copolymer ligands to afford water dispersible AuNP@PS-b-PAA, where the poly(acrylic acid) segments were dissolved in water (Fig. 10a,b). Structural transformation of polymer micelles from spheres to cylinders was induced by addition of $\mathrm{HCl}$ to warm $\left(70{ }^{\circ} \mathrm{C}\right)$ AuNP@PS- $b$-PAA dispersions in DMF/water (7:3 v/v), which resulted in the assembly of block copolymer encapsulated AuNPs (Fig. 10c). Assemblies obtained using these DMF/water mixtures consisted of two laterally associated linear AuNP chains ("double-line chains"). The formation of these laterally associated assemblies was attributed to the aggregation of the coreshell NPs and subsequent reorganization of PS- $b$-PAA shells to form cylindrical micelles, and the colloidal polymers were preferentially isolated by centrifugation (Fig. 10d).[180]

\section{INSERT FIGURE 10 AND FIGURE 10 CAPTION}

Chen and coworkers used the same system described above to further demonstrate precise control of colloidal monomer lateral association into colloidal polymers by variation of solvent mixtures (DMF/ $\mathrm{H}_{2} \mathrm{O}$, Fig. 10).[180] The formation of block copolymer stabilized colloidal polymers possessing "double-line chains" were found to be more thermodynamically stable than linear AuNP chains which were not laterally associated ("single-line chains"). Singleline chains obtained in $\mathrm{DMF} /$ water $(6: 1 \mathrm{v} / \mathrm{v})$ were readily transformed to double line chains after treating colloids in dispersions with increased water content (DMF/water 7:3 v/v), while the reversion of double-line chains to single-line chains was not observed. Double-line chains possessing degrees of polymerization up to $200 \mathrm{NPs}$ were observed, while single-line chains 
resulted in colloidal polymers of slightly higher DP (300 NPs). Additionally, the authors utilized larger NPs as "colloidal initiators" by introducing 30 or $40 \mathrm{~nm}$ AuNPs or $45 \mathrm{~nm}$ Ag nanocubes as seeds to grow chains composed of two laterally bundled NPs, so that the terminal starting points could be identified. The polymer shells of these colloidal initiators were destabilized by the addition of $\mathrm{HCl}$, but these larger NPs did not aggregate due to their low concentration. Upon the addition of an excess of smaller AuNP@PS- $b$-PAA $(\mathrm{D}=16 \mathrm{~nm})$ colloidal monomers, larger NPs were observed preferentially at the end of the resulting double-line chains (Fig. 10e-g).

Murphy and coworkers demonstrated the formation of linear colloidal homopolymers and statistical copolymers of various CTAB-capped AuNP morphologies assembled on TEM grids through interactions of hydrophobic domains at NP termini (Fig. 11).[168] This study revealed that colloidal monomers having sharp edges (e.g., NRs, bipyramids, and "spiked" NPs; Fig. 11a,c) were prone to the formation of linear assemblies with random monomer connectivity, despite being functionalized by a single type of small molecule ligand.[168] Spherical AuNPs were found to form assemblies with more branched architectures (Fig. 11b). The observed assembly behavior was attributed to the organization of CTAB ligands as bilayer assemblies on AuNR lateral facets.[168] Intuitively, CTAB bilayers on a colloidal surface would be expected to present positively charged head-groups to the solution enabling aqueous dispersions of AuNRs. However, since these bilayers were less stable on nanorod termini surfaces due to increased curvature, the CTAB monolayers presented a greater fraction of hydrophobic alkyl chains to the aqueous medium. Thus, sharp NP facets (such as NR termini or tips of bipyramids) associated through hydrophobic interactions of these alkyl chains at the AuNR termini in polar solvents. This system was an early example of linear AuNR assembly through association of differentially functionalized "patchy particles,"[168] as predicted by molecular simulations.[167] Nanoparticle 
sizes and colloidal polymer DP were not discussed for the linear assemblies in this report.[168] Lyotropic materials have also been synthesized which relied upon differential ligand binding to direct the assembly of AuNRs.[181]

\section{INSERT FIGURE 11 AND FIGURE 11 CAPTION}

Wang and Yan and coworkers devised an approach to the formation of quasi-alternating plasmonic colloidal copolymers based on zwitterionic interactions between ligands on the termini of AuNRs and bipyramids (Fig. 12).[34] In this system, CTAB-coated AuNRs and bipyramids were preferentially functionalized with either glutathione or cysteine at the terminal facets, and linear assemblies were proposed to form through complementary interactions of these zwitterionic endgroups (Fig. 12a,b). Colloidal copolymers with quasi-alternating monomer connectivity were observed when AuNRs ( $D \approx 14 \mathrm{~nm}$, Fig. $12 \mathrm{c}, \mathrm{d}$ ) or bipyramids ( $\mathrm{D} \approx 45 \mathrm{~nm}$, Fig. 12e,f) were blended with AuNPs of comparable diameter ( $D=14 \mathrm{~nm}$ or $45 \mathrm{~nm}$, respectively) in the presence of glutathione or cysteine. The length of these linear assemblies (DP), and the mechanism resulting in the formation of quasi-alternating copolymers (versus blocks or homopolymers) were not discussed, though the results suggested that reactivity ratios[22] of AuNPs with Au rods or pyramids were small to explain the obtained results $(\mathrm{r}<<1)$ . Binary self-assembly of AuNPs and AuNRs with gold nanowires was also investigated in related work by Liz-Marzán and coworkers[182]

INSERT FIGURE 12 AND FIGURE 12 CAPTION 
Kumacheva and coworkers enhanced the "patchiness" of CTAB coated AuNRs by the functionalization of nanorod termini with thiol-terminated polystyrene ligands, which enabled the formation of linear and cyclic colloidal polymers when dispersed and deposited onto substrates from selective solvent systems.[169] Association of these polystyrene ligands in various solvent mixtures was utilized to afford reversible and highly tunable access to colloidal polymer architectures through hydrophobic interactions of PS ligands at nanorod termini.[183] In general, the formation of colloidal polymer assembles was conducted with dilute dispersions of AuNRs $(\mathrm{L}=40 \mathrm{~nm}, \mathrm{D}=10 \mathrm{~nm})$ in DMF $(0.0075 \mathrm{mg} / \mathrm{mL}$ AuNRs $)$ that were end-functionalized with thiol-terminated PS ligands where the addition of water promoted colloidal polymerization through hydrophobic associations of the PS ligands endcapped onto NR termini (see Fig. 13 for representative example with varying molar mass of PS ligands).[184]

For this patchy AuNR system, Kumacheva and coworkers also investigated the effect of molecular weight of terminal PS ligands on colloidal polymer architecture, where the use of higher molar mass PS ligands correlated with the formation of linear and laterally bundled AuNR assemblies (Fig. 13a-d).[184] A phase diagram was constructed for varying water concentrations and PS ligand $\mathrm{M}_{\mathrm{n}}$ which mapped conditions for obtaining rings, chains, bundles, and bundled chains (double-line chains). Furthermore, variation of PS ligand molar mass was also observed to correlate with the interparticle distances between NP units within colloidal polymer chains as determined by UV-vis spectroscopy and TEM studies (Fig. 13e-g). Degrees of polymerization of up to $50 \mathrm{NPs}$ (as observed by TEM) were obtained for linear chains of AuNRs endfunctionalized with PS ligands $\left(\mathrm{M}_{\mathrm{n}}=30,000 \mathrm{~g} / \mathrm{mol}\right)$ cast from DMF/water dispersions .[184]

\section{INSERT FIGURE 13 AND FIGURE 13 CAPTION}


AuNRs $(\mathrm{L}=43 \mathrm{~nm}, \mathrm{D}=12 \mathrm{~nm})$ functionalized at both termini with thiol terminated poly(N-isopropylacrylamide) (PNIPAm) ligands were also demonstrated to undergo (photo)thermally-triggered self-assembly to form linear and branched colloidal polymers (Fig. 14).[185] AuNRs were heated above the lower critical solution temperature (LCST) of PNIPAm $\left(M_{n}=13000 \mathrm{~g} / \mathrm{mol}\right)$ to induce colloidal polymer formation (Fig. 14b,c). The average DP was reported to be 11 NR units per chain using this LCST approach to colloidal polymer formation. Optical spectroscopy was utilized to confirm the presence of assemblies in the dispersed state, to complement imaging of AuNR samples deposited onto supporting substrates . The 1-D selfassembly of PNIPAm functionalized AuNRs was also observed upon illumination at a wavelength near the longitudinal plasmon band of the NRs due to optically induced heating to trigger the LCST of PNIPAm chains (Fig. 14a).[185]

\section{INSERT FIGURE 14 AND FIGURE 14 CAPTION}

The colloidal polymerization of polystyrene-terminated AuNRs ( $\mathrm{L}=50 \mathrm{~nm}, \mathrm{D}=12 \mathrm{~nm})$ was later shown to follow step-growth polymerization models, as tracked by the optical plasmon absorbance of NR monomers (Fig. 15).[186] This mechanistic study was perhaps the first quantification of supramolecular nanorod assembly using classical polymerization approaches .[22] Furthermore, polymerization parameters related to DP and other NR bonding considerations (e.g., cis/trans isomerism, cyclization, branching, interparticle angles) were also measured by TEM imaging and incorporated into the theoretical description of AuNR assembly.[186] The polydispersity of this system was found to approach a value of 2 at high 
conversion of AuNR colloidal monomers (tracked up to DP $=16$ ), which was in agreement with classical step-growth polymerization predictions (Fig. 15d).[186]

\section{INSERT FIGURE 15 AND FIGURE 15 CAPTION}

Similar to the previous work on the self-assembly of AuNPs, the ionic strength of NP dispersions was later shown to be a useful parameter for controlling the colloidal polymerization of polystyrene end-functionalized AuNRs.[187] However, the major driving force for selfassembly in the case of PS functionalized AuNRs was found to be a decrease in solubility of the polystyrene ligands, rather than screening of electrostatic repulsion previously observed for AuNPs. Photocrosslinking of poly(styrene-co-isoprene) end-functionalized AuNRs was also demonstrated as a useful means to covalently fix the bond angles in AuNR colloidal polymers,[188] and plasmonic properties of related systems were later correlated with colloidal polymer structure.[189-193]

$\mathrm{Au}-\mathrm{Fe}_{3} \mathrm{O}_{4}$ heterodimers were recently utilized to prepare $\alpha, \omega$-telechelic colloidal polymers by end-capping AuNR colloidal polymers with $\mathrm{Au}-\mathrm{Fe}_{3} \mathrm{O}_{4}$ heterodimers (Fig. 16a).[194] In this case, the $\mathrm{Au}-\mathrm{Fe}_{3} \mathrm{O}_{4}$ heterodimers served as colloidal analogs to end-capping agents (or B' agents) in the colloidal polymerization of AuNRs. These $\mathrm{Au}-\mathrm{Fe}_{3} \mathrm{O}_{4}$ heterodimers $\left(\mathrm{D}_{\mathrm{Au}}=6.5 \mathrm{~nm}, \mathrm{D}_{\mathrm{Fe} 3 \mathrm{O} 4}=15.4 \mathrm{~nm}\right)$ were synthesized based on the work of Sun and coworkers, $[14]$ and the Au domains were functionalized with thiol-terminated PS ligands (Fig. 16c).[194] AuNRs (L = $35 \mathrm{~nm}, \mathrm{D}=10 \mathrm{~nm}$, Fig. 16b) functionalized with PS ligands at nanorod termini were then blended with these heterodimers in DMF/water mixtures (15\% water v/v). The PScoated AuNP segments on $\mathrm{Au}-\mathrm{Fe}_{3} \mathrm{O}_{4}$ NPs were able to associate with PS-coated nanorod termini, 
where these heterodimers were utilized as a monofunctional B' colloidal monomer, or "chain stopper" (Fig 16d vs. 16e). The relative molar ratio of chain stoppers to AuNRs was varied from $0-1.55$, which enabled the preparation of colloidal polymers of controllable DP.[194]

\section{INSERT FIGURE 16 AND FIGURE 16 CAPTION}

Both statistical and segmented colloidal copolymers were recently prepared for CTAB coated plasmonic ( $\mathrm{Au}$ and Pd) nanorods end functionalized with PS ligands in DMF/water mixtures (Fig. 17).[195] AuNRs of differing lengths ( $\mathrm{L}=50$ and $80 \mathrm{~nm}$ ) and comparable diameters $(12 \mathrm{~nm})$ were found to have different reactivity towards homo or cross nanoparticle coupling reactions. Shorter nanorods $(\mathrm{L}=50 \mathrm{~nm})$ were observed to self-associate at a faster rate than coupling of shorter nanorods with longer nanorods $(\mathrm{L}=80 \mathrm{~nm})$ under these conditions. Hence, due to the differential reactivity of nanorods as a function of size, statistical colloidal copolymers were prepared by controlling the feed ratio of the respective comonomers, where the monomer incorporation was tracked by scanning TEM (STEM) (Fig. 17a). This approach was extended to include PdNR comonomers $(\mathrm{L}=183 \mathrm{~nm}, \mathrm{D}=19 \mathrm{~nm}$ ) to afford colloidal copolymers of tunable composition.

Both statistical and segmented copolymers were obtained for mixtures of Au and Pd NRs in the same report.[195] Segmented, or block, copolymers were prepared using classical prepolymer approaches,[22] where preformed colloidal homopolymers of NRs were blended in situ to form blocky copolymers (Fig. 17b). The authors also showed that the less reactive PdNR colloidal monomers could be added to preformed AuNR chains as an alternative approach to segmented colloidal copolymers. These colloidal (co)polymers were oligomeric with average DP 
$\approx 10$ NRs, though chains consisting of up to 17 NRs were also observed (based on STEM images).

\section{INSERT FIGURE 17 AND FIGURE 17 CAPTION}

Kumacheva and coworkers also demonstrated that AuNP-tipped CdSe nanorods could be treated as colloidal analogs to molecular amphiphiles to access a variety of self-assembled structures including stars and oligomeric brushes (denoted as "elongated objects" in Fig. 18a).[196] CdSe nanorods having a range of lengths $(\mathrm{L}=8-28 \mathrm{~nm})$ and comparable diameters $(3-4 \mathrm{~nm})$ were investigated, where nanorods incorporated a single AuNP tip $(\mathrm{D}=5 \mathrm{~nm})$. The CdSe nanorods were capped with $\mathrm{C}_{12}$ terminal thiol ligands, while the AuNP tips were coated with didodecyldimethylammonium bromide and 1-dodecylamine ligands.[196] The selfassembly of these nanorods was thus controlled by tuning both the relative volume fractions of the $\mathrm{Au}$ and $\mathrm{CdSe}$ segments and the quality of solvent for each of the segments, which enabled access to a range of self-assembled morphologies (Fig. 18a). Oligomeric "elongated objects" containing approximately 10 NPs (based on STEM images) were obtained (Fig. 18b,c), where the structural evolution of self-assembled morphologies was tracked by STEM and dynamic light scattering experiments.

\section{INSERT FIGURE 18 AND FIGURE 18 CAPTION}

Silver nanoparticles (AgNPs) have also been reported to form plasmonic assemblies through non-covalent interactions, as was recently reviewed.[197] High aspect ratio (large DP) 
colloidal polymers were obtained through non-covalent interactions of Ag-thiolate polymers (Ag(I) and tiopronin ligands) and AgNPs via an ultra-sonication approach.[198] A colloidal suspension of tiopronin-stabilized AgNPs $(\mathrm{D}=16 \mathrm{~nm})$ was dried and the solid product was redispersed in deionized water under ultrasound sonication before preparing a sample for TEM studies. Colloidal polymers consisting of smaller $(\mathrm{D}=4 \mathrm{~nm})$ colloidal monomers were obtained from sonication of larger $(\mathrm{D}=16 \mathrm{~nm})$ AgNP dispersions, [198] where the change in particle diameter was proposed to occur due to digestive ripening of larger $(16 \mathrm{~nm})$ precursors.[199] The formation of 1-D colloidal polymers was attributed to the presence of $\mathrm{Ag}(\mathrm{I})$-tiopronin coordination polymers, where linear NP assemblies were held together by a combination of hydrogen bonding, van der Waals forces, and argentophilic interactions. Furthermore, the possibility of (ligand induced) dipolar interactions assisting in the formation of colloidal polymers could not be excluded for this system under these conditions. However, this metalthiolate mechanism for colloidal polymer formation was extended to include AuNPs by using $\mathrm{Cu}(\mathrm{I})$ and $\mathrm{Au}(\mathrm{I})$-thiolates to form linear thiolate structures in the same report.[198] The resulting linear assemblies of both AgNPs and AuNPs based on thiolate templates spanned several microns in length, but the average DP of these colloidal polymers was not explicitly discussed. An example of controlling colloidal polymer structure by non-covalent interactions was demonstrated for silver nanocubes (AgNCs), where both edge-edge and face-face orientations of colloidal monomers were accessible within linear colloidal polymers formed in a PS matrix (Fig. 19).[200] AgNCs (80 nm) were capped with polymer ligands (thiol-terminated PEG or PVP) and then cast in polystyrene thin films, where entropy-driven depletion demixing resulted in 1-D nanoparticle association.[201] Linear colloidal polymers (as opposed to the formation of 2-D networks) consisting of up to $100 \mathrm{AgNCs}$ were proposed to occur due to a combination of steric 
effects,[202] and diffusion-limited aggregation.[203] The additional enthalpic interactions between polymer ligands bound to neighboring AgNCs were then utilized to control colloidal monomer orientation to obtain either kinetically trapped (edge-edge) orientations or more thermodynamically favorable (face-face) orientations (Fig. 19a). PVP-coated nanocubes were observed to predominantly retain their initial edge-edge orientations after annealing, while PEGcoated AgNCs were found to reorganize into more diverse 1-D arrangements depending on the thermal history of the thin film (Fig. 19b,c). The molar mass of surface bound polymer ligands was also utilized to control colloidal monomer orientation for $\mathrm{AgNCs}$, based on predictions obtained from Monte Carlo simulations.[200]

\section{INSERT FIGURE 19 AND FIGURE 19 CAPTION}

\section{Surface-directed formation of colloidal polymers}

Surface-directed colloidal polymerzation is defined as the 1-D aggregation of colloidal monomers through irreversible associations or bonding between NP inorganic surfaces, resulting in linear or lightly branched self-assembled architectures. To date, only a few systems have been demonstrated to form colloidal polymeric assemblies through a surface-directed mechanism, herein described as oriented attachment or nanowelding approaches.

\subsection{Oriented attachment}

Oriented attachment mechanisms for colloidal polymer formation occur via the selfassembly of NPs to form larger single crystals by sharing of common crystallographic orientations resulting in linear, cyclic, or network NP assemblies.[204-208] The mechanism governing aggregation and coalescence of NPs via oriented attachment is currently under study 
for a variety of colloidal polymer systems.[208-209] For purposes of this review, it is interesting to note that the initial organization of these colloidal monomers has been shown to follow stepgrowth type polymerization kinetics.[210] Oriented attachment has been extensively studied for a wide variety of metal (e.g., Ag, [211-212] Au,[213] Pt[214]), metal oxide (e.g., $\mathrm{TiO}_{2}$,[204-206, 215] $\mathrm{ZnO}[216]$ ), and metal chalcogenide (e.g., CdSe,[217] $\mathrm{PbSe}$ [35]) NPs, and the reader is referred elsewhere for comprehensive reviews on this subject.[209, 218]

Murray and coworkers demonstrated the synthesis of a variety of polymeric architectures using PbSe nanocrystalline colloidal monomers via oriented attachment colloidal polymerizations (Fig. 20).[35] In this system, stabilizing ligands used during the synthesis of PbSe NPs affected the growth and resulting end morphology of colloidal monomers by altering the surface energy of various nanocrystal facets. The morphology of these PbSe NPs (colloidal monomers) then controlled the structure of the resulting colloidal polymers through oriented attachment of exposed high energy facets. The initial linear assembly of PbSe NPs was attributed to the inherent charge dipole present in cubic PbSe along the $\langle 100\rangle$ axis, followed by oriented attachment of the constituent NPs. However, although both dipolar assembly and oriented attachment contributed to the formation of these PbSe colloidal polymers, the control of chain length and architecture demonstrated in this early example by Murray and coworkers required NP fusion to form chemically linked structures through sharing of common crystallographic orientations (e.g. oriented attachment).[35] Single-crystal PbSe nanowires and nanorings ranging from 4-20 nm in diameter and up to $30 \mu \mathrm{m}$ in length were synthesized in the presence of oleic acid, hexadecylamine, trioctylphosphine, and/or $n$-tetradecylphosphonic acid at reaction temperatures ranging from $190-250{ }^{\circ} \mathrm{C}$. 


\section{INSERT FIGURE 20 AND FIGURE 20 CAPTION}

In this $\mathrm{PbSe}$ colloidal polymer system, Murray and coworkers, proposed that nanowire (NW) formation occurred by both dipolar assembly and oriented attachment of PbSe colloidal monomers having a rock salt crystal structure (Fig. 20a), which were obtained using a combination of oleic acid and $n$-tetradecylphosphonic acid stabilizing ligands.[35] Long-chain, aliphatic primary amines induced the formation of octahedral PbSe nanocrystals terminated by eight $\{111\}$ facets which were preferentially eliminated during self-assembly to form nanowires with zigzag morphologies through sharing of $\{111\}$ faces (Fig. 20b). Conversely, branched, starshaped, PbSe NPs were obtained by optimization of the reaction conditions leading to octahedral $\mathrm{PbSe}$ nanocrystals using primary amines at high $\mathrm{Pb}$ concentration. Linear assembly and oriented attachment of these branched colloidal monomers resulted in the formation of radially branched wires resembling bottlebrush polymers with a high grafting density of side chains (Fig. 20c). Finally, the formation of cyclic PbSe colloidal polymers ("nanorings") was demonstrated for cubic PbSe colloidal monomers synthesized in the presence of hexadecylamine at higher growth temperatures ( $\mathrm{T}>200^{\circ} \mathrm{C}$, Fig. 20d). Similar cyclic colloidal polymers were obtained by Zeng and coworkers for CdS NPs by an oriented attachment mechanism.[219] This example by Murray and coworkers demonstrated the precise control of colloidal polymer architecture which can be obtained from oriented attachment by variation of colloidal monomer structure and reactivity.[35]

\subsection{Nanowelding}

Nanowelding is a recently coined term by Manna and coworkers that describes the fusion of preformed NPs through direct contact of inorganic domains to form a new, larger, chemically 
bonded structure (Fig. 21).[36] These structures were primarily branched,[36] but in principle could form linear colloidal polymers with the appropriate design of colloidal monomers. The work of Manna and coworkers utilized shape anisotropic semiconductor NPs of various morphologies ("bullets", nanorods, and tetrapods) which were functionalized with one or more AuNP tips. Colloidal polymerization was then induced by destabilization of surface ligands on the gold domains by the addition of molecular iodine to NP dispersions.[36] Coalescence of these exposed gold domains resulted in branched colloidal polymers, with the degree of branching depending on the number of semiconductor domains per AuNP-junction (Fig. 21a). These coalesced AuNP tips were observed to be polycrystalline after nanowelding (Fig. 21h,i).

\section{INSERT FIGURE 21 AND FIGURE 21 CAPTION}

In the system of Manna and coworkers,[36] the morphology of the semiconductor component was utilized to dictate the number of polymerizable moieties (AuNP-tips) per colloidal monomer, which enabled the formation of extended networks (Fig. 1f, CdTe@CdS tetrapods, $\mathrm{L}_{\text {arm }} \approx 20-50 \mathrm{~nm}, \mathrm{D}_{\text {arm }} \approx 5 \mathrm{~nm}$ ), branched chain-like assemblies (Fig. 21f-i, CdSe nanorods, $\mathrm{L}=80 \mathrm{~nm}, \mathrm{D}=4 \mathrm{~nm}$ ), and propeller-like structures (Fig. 21b-e, bullet shaped NPs, L $=30 \mathrm{~nm}, \mathrm{D}=20 \mathrm{~nm})$ joined by metallic AuNP-tip junctions $(\mathrm{D} \approx 3-5 \mathrm{~nm})$.[36] The chain length and DP of colloidal polymers formed after nanowelding were not explicitly discussed, although the authors noted that colloidal polymers composed of AuNP-tipped CdSe nanorods consisted of several tens of NPs.[36] Similar network colloidal polymer architectures were later obtained by thermal treatment of AuNP-tipped nanorod dispersions, which offers an alternative approach to using molecular iodine for nanowelding approaches.[220] Although nanowelding methodologies have not yet been demonstrated to enable the synthesis of purely linear colloidal 
polymers, the possibility remains that a balance between steric considerations of nanorod segments and AuNP-tip diameter could be found to provide access to polymeric architectures . Nanowelding approaches are anticipated to be a more general methodology to polymerize other NP types as more systems and colloidal monomers are developed.

\section{Dipole-directed formation of colloidal polymers}

The term dipole-directed colloidal polymerization refers to the formation of linear (or lightly branched) assemblies from interactions of charge or spin dipoles within inorganic cores of disparate NPs. The dipole-directed formation of colloidal polymers is sensitive to changes in the composition and crystal phase of the material, where these attractive interparticle dipolar forces can be mitigated by ligand-induced steric repulsions or thermal fluctuations. It is important to note that these dipoles are intrinsic to the inorganic NP core and differ from ligand-induced dipoles arising from incorporation of certain organic ligands (such as thiols on AuNPs, mentioned in section 2.1 above) on the colloid surface. Additionally, both dipolar and van der Waals interactions are present in dipole-directed colloidal polymerization systems, and the architecture of the resulting colloidal polymers is dependent upon the balance between these two attractive forces.

An elegant series of 2-D simulations by Benkoski and Douglas and coworkers reported on the competition between dipolar and van der Waals effects in colloidal assembly, . (Fig. 22),[221] where a landscape of possible self-assembled morphologies were formed from thermal energy, van der Waals forces (x-axis), and/or dipolar interactions (y-axis).[221-223] In this simulation, particles were dispersed into a 2-D gas when all attractive interparticle interactions were exceeded by thermal energy (Fig. 22T). In the absence of significant van der Waals forces, increasingly linear self-assembled structures were observed as dipolar interactions exceeded 
thermal fluctuations (Fig. 22d1-d3). Conversely, increasing van der Waals forces in the absence of dipolar interactions resulted in the formation of fractal assemblies through reaction and diffusion limited cluster aggregation (Fig. 22v1-v3). Dendritic assemblies were also observed when both van der Waals forces and dipolar interactions simultaneously exceeded thermal energy (Fig. 22dv1-dv4).[221]

\section{INSERT FIGURE 22 AND FIGURE 22 CAPTION}

\subsection{Electrostatic dipoles}

CdTe NPs composed of the cubic zinc blend phase have sufficiently strong electrostatic dipoles to promote the formation of linear assemblies, where the mechanistic aspects of the polymerization process have been extensively investigated. ${ }^{28,99,201-203}$ Ligand-induced repulsive interactions were often minimized by removal of excess ligands from NP dispersions prior to colloidal polymer formation.

Kotov and coworkers demonstrated the formation of linear and branched assemblies of CdTe NPs from ligand depleted dispersions and the subsequent transformation of these NP chains into luminescent CdTe nanowires (CdTe NWs, Fig. 23).[37] Thioglycolic acid capped zinc blend CdTe NPs $(\mathrm{D}=2.5-5.4 \mathrm{~nm})$ were synthesized based on the methods of Rogach and coworkers[224-225] and were then purified to remove excess ligands present after the NP synthesis.[37] This purification step was required to enable colloidal polymerization of CdTe colloidal monomers, where the authors noted that the dipole moments of CdTe NPs were not affected by depletion of surface bound ligands. The obtained aqueous NP dispersions were then allowed to age in the dark at room temperature for up to 48 hours to form "pearl-necklace" 
agglomerates consisting of CdTe NPs (Fig. 23a,b).[37] Continued aging of these aqueous NP dispersions resulted in the formation of linear, single-crystalline, wurtzite, CdTe NWs exhibiting lengths exceeding one micron, where the individual CdTe NPs $(D=3.4$ or $5.5 \mathrm{~nm})$ were no longer distinguishable by TEM (Fig. 23c).[37] Furthermore, single crystalline CdTe NW diameters correlated closely with the diameter of the initial CdTe NPs prior to self-assembly. These findings suggested that the NWs formed due to the recrystallization of the linearly assembled CdTe NPs to form rigid nanowires, which were luminescent as observed by confocal microscopy (Fig. 23d-g).[37]

\section{INSERT FIGURE 23 AND FIGURE 23 CAPTION}

Later theoretical and experimental investigations of these CdTe systems by Kotov and coworkers revealed the initial NP assembly to be dependent on a synergistic combination of electrostatic dipolar, van der Waals, and hydrophobic attractions balanced by electrostatic repulsion from surface bound ligands.[30, 37, 226-228] Monte Carlo simulations suggested that CdTe NPs in these colloidal polymers were arranged in a face-to-face orientation, with dipole directions alternating perpendicular to the wire axis (as opposed to a head-to-tail orientation).[227] Kotov and coworkers also demonstrated that the recrystallization process of nanowire formation from CdTe and CdSe NPs could be manipulated to produce Te and Se NWs (respectively) by the addition of EDTA as a Cd-complexing agent during aging of liganddepleted NP dispersions.[229]

More recently, colloidal copolymers of CdTe NWs were prepared from the recrystallization and Ostwald ripening of CdTe NPs within chains (Fig. 24).[230] Kotov and 
coworkers demonstrated the formation of nanowires with corrugated features by blending $\mathrm{CdTe}$ NPs having diameters of $2.4 \mathrm{~nm}$ and $5.8 \mathrm{~nm}$, followed by AFM interrogation of the selfassembled structures (Fig. 24).[230] CdTe NWs formed from binary blends of CdTe NPs (copolymers) were found to have diameters which varied significantly more than for NWs prepared from either of the constituent NP diameters (homopolymers). Presumably, this blending methodology can be further utilized to include nanowires of gradient or blocky compositions , and could enable the incorporation of pendant functionality if additional NP domains were carried into the assembly.

\section{INSERT FIGURE 24 AND FIGURE 24 CAPTION}

A number of other colloidal polymers have been prepared from these semiconductor (CdTe) colloidal monomers followed by NP conversion reactions. Kotov and coworkers demonstrated the preparation of $\mathrm{Ag}_{2} \mathrm{Te} \mathrm{NW}$ networks by cation exchange reactions from preformed CdTe NWs, where the ligand density on NPs was again a crucial parameter affecting the final colloidal polymer architecture.[231] Silica coating by post-functionalization of CdTe nanowires resulted in bristled "nano-centipedes",[232] which resembled classical polymer brushes,[233] and CdTe chain formation was also investigated under conditions which disfavored nanowire formation.[234] Related studies have demonstrated that CuS NPs selfassembled to form ribbons,[235] while CdTe and CdS nanoparticles formed twisted ribbons under illumination.[236] $\mathrm{ZnO}$ hexagonal pyramids represented a relatively new class of colloidal monomer, which have been demonstrated to form oligomeric collodial polymers.[237] 


\subsection{Magnetic dipoles}

Magnetic dipolar nanoparticles are known to form linear chains under zero-field conditions (i.e., in the absence of an applied external field) due to interparticle interactions of the magnetic spin dipoles in each colloidal monomer.[47-48, 238-241] Examples of these systems have been observed for $\mathrm{Fe}_{3} \mathrm{O}_{4}$ colloids in magnetotactic bacteria,[53] and magnetic dipolar NPs have most commonly been observed using either (bi)metallic ferromagnetic or metal oxide ferrimagnetic colloids.[41, 242] Early work by Thomas and Hess and coworkers demonstrated the spontaneous formation of linear NP chains from polymer stabilized cobalt nanoparticles (CoNPs).[51-52] Similar dipolar NP systems were later developed using polymeric ligands to enhance the colloidal stability of dipolar magnetic NPs.[243-248] Uniformly sized ferromagnetic nanoparticles have also been prepared from thermolysis reactions of metal carbonyls in the presence of small molecule ligands (e.g., oleic acid, oleylamine, and trioctylphosphine oxide).[249-251]

The assembly process by which magnetic dipolar NPs associate is similar to step-growth polymerization of A-B monomers.[22] These systems have been described using equilibrium polymerization models, where stronger dipolar coupling (relative to $-\mathrm{k}_{B} \mathrm{~T}$ ) favors the formation of NP chains over freely dispersed monomers.[252] Although the formation of linear assemblies from magnetic NP colloidal monomers has been extensively studied, control of key polymer parameters such as DP, composition (copolymers), and architecture have not been widely investigated for magnetic dipole-induced colloidal polymers. Previous examples demonstrating control of colloidal polymer parameters have generally utilized CoNP colloidal monomers, as discussed in the examples below. 
A key example of controlling colloidal polymer structure by magnetic dipolar assembly was the formation of nanoparticle bracelets, as pioneered by Wei and coworkers for CoNPs using modified resorcinarene ligands (Fig. 25).[38, 42, 46, 49, 253-254] Cobalt nanoparticles (D $=27 \mathrm{~nm}$ ) were synthesized by the thermolysis of $\mathrm{Co}_{2}(\mathrm{CO})_{8}$ in refluxing toluene containing $\mathrm{C}$ undecylcalix[4]resorcinarene tetraphosphonite ligands to afford dipolar bracelets, or "flux closure rings," consisting of approximately 5 - 12 NPs (Fig. 25a,b).[38, 253] The formation of these cyclic assemblies was attributed to the dipolar coupling of exposed chain ends possessing opposite north-south magnetic poles (Fig. 25c-f). Open linear chains were more frequently observed at ligand concentrations higher or lower than the optimal $10^{-4} \mathrm{M}$, which was attributed to the delicate balance required between dipolar interactions, van der Waals interactions, and the viscosity of the dispersion during drying of samples on carbon coated TEM grids. Other examples of dipolar magnetic NP bracelets have been reported using a variety of ligands, which demonstrated the generality of various methods to access to cyclic colloidal polymer architectures.[255-259]

\section{INSERT FIGURE 25 AND FIGURE 25 CAPTION}

Pyun and coworkers developed the synthesis of dipolar CoNPs $(D \approx 20 \mathrm{~nm})$ functionalized with end-functionalized polystyrene ligands $\left(\mathrm{M}_{\mathrm{n}} \approx 10,000 \mathrm{~g} / \mathrm{mol}\right)$, which spontaneously formed linear colloidal polymers.[260-263] This methodology was modified to enable the large scale synthesis of dipolar PS-coated CoNPs, which could be readily isolated and stored as powders prior to redispersion in organic solvents.[264] The assembly and shape persistence of colloidal polymers made from dipolar PS-CoNPs were then extensively studied by 
Pyun and Benkoski and coworkers on surfaces, at oil/water interfaces, and in solution.[44, 221, 265-267]

The fabrication of microscopic "artificial swimmers" consisting of a chain of approximately $70 \mathrm{CoNPs}(\mathrm{D}=24 \mathrm{~nm})$ attached to a single, larger, $\mathrm{Fe}_{\mathrm{x}} \mathrm{O}_{\mathrm{y}}$ bead $(\mathrm{D}=250 \mathrm{~nm})$ was demonstrated by blending these colloids in an external magnetic field. These binary assemblies were held together solely by dipolar associations.[266] Actuation of these artificial flagella (dispersed in DMF) by an oscillating external field and directional progression of swimmers were observed using differential interference contrast optical microscopy.[266] The fabrication of colloidal polymer brush-like surfaces capable of artificial cilia-type actuation was also reported.[267]

Magnetic-dipole induced colloidal polymers have also been chemically modified in situ to form chemically bonded nanowires by the oxidation of CoNPs with chalcogens $(E=\mathrm{O}, \mathrm{S}, \mathrm{Se}$, Te). This approach was based on earlier reports by Alivisatos and coworkers for individual CoNPs to afford larger, hollow, $\mathrm{Co}_{\mathrm{x}} \mathrm{E}_{\mathrm{y}} \mathrm{NPs}$ via the nanoscale Kirkendall effect.[268-271] The nanoscale Kirkendall effect was utilized by Xu and coworkers to convert chains of CoNPs from supramolecular magnetic assemblies to chemically bonded colloidal polymers by dimensional expansion of closely associated dipolar CoNPs during chalcogenation.[272] CoNPs $(D \approx 20 \mathrm{~nm})$ were converted into micron long $\mathrm{Co}_{x} \mathrm{E}_{\mathrm{y}}$ nanowires $(\mathrm{E}=\mathrm{S}, \mathrm{Se}, \mathrm{Te})$ by the addition of chalcogen precursors to CoNP dispersions in refluxing 1,2-dichlorobenzene, and dipolar assemblies were captured in solution by the dimensional expansion due to the nanoscale Kirkendall effect. Dipolar ferromagnetic CoNPs were shown to be necessary for the formation $\mathrm{Co}_{x} \mathrm{E}_{\mathrm{y}} \mathrm{NWs}$ in the absence of an external magnetic field, as superparamagnetic CoNPs formed only individual $\mathrm{Co}_{x} \mathrm{E}_{\mathrm{y}}$ NPs after chalcogenation.[272] This, to our knowledge, was one of the first examples of a 
colloidal polymerization that harnessed both dipolar assembly of CoNPs and a NP conversion reaction to form chalcogenide nanowires. Related work on galvanic exchange[273-276] was utilized for the formation of hollow noble metal NWs, [277] along with alloying reactions using $\mathrm{Sb}^{3+}$ precursors for the formation of $\mathrm{CoSb}_{3} \mathrm{NWs}$.[278]

Pyun and coworkers reported on the solution oxidation of polystyrene-capped CoNPs and concomitant dimensional expansion of CoNPs resulted in micron sized $\mathrm{Co}_{\mathrm{x}} \mathrm{O}_{\mathrm{y}} \mathrm{NWs}$ due to the nanoscale Kirkendall effect in a process termed colloidal polymerization (Fig. 26).[279] The modularity of this colloidal polymerization approach was also demonstrated by a variety of polymer pre- and post-functionalization strategies which incorporated noble metals (e.g., Au, Pt) into either the colloidal polymer core or periphery for enhanced electrochemical activity.[280283] Kim and Pyun and coworkers prepared Au@CoNPs $(\mathrm{D}=22 \mathrm{~nm})$ by deposition of cobalt shells onto preformed AuNP seed particles $(\mathrm{D}=13 \mathrm{~nm})$, which then spontaneously formed linear assemblies due to spin dipolar interactions of the ferromagnetic cobalt shells.[280] Oxidation of these Au@CoNP assemblies resulted in the formation of micron sized $\mathrm{Co}_{\mathrm{x}} \mathrm{O}_{\mathrm{y}} \mathrm{NWs}(\mathrm{D}=31 \mathrm{~nm})$ which incorporated intact AuNP cores into each monomeric unit (Fig. 26a). The AuNP cores were clearly resolved by TEM imaging of these $\mathrm{Au} @ \mathrm{Co}_{\mathrm{x}} \mathrm{O}_{\mathrm{y}}$ colloidal polymers and comparison with $\mathrm{Co}_{\mathrm{x}} \mathrm{O}_{\mathrm{y}}$ nanowires synthesized from CoNPs without AuNP cores (Fig. 26b,c).[280]

\section{INSERT FIGURE 26 AND FIGURE 26 CAPTION}

Magnetic NP-semiconductor nanorod heterostructures have been synthesized for several systems as a route to materials with novel magneto-optical and catalytic properties.[284-292] However, the magnetic NP domains (or "tips") on these heterostructured nanorod systems were 
generally not strongly dipolar, and thus did not form colloidal polymers. By contrast, highly

branched colloidal polymer structures were obtained from dipolar assembly of cobalt domains (D $\approx 15 \mathrm{~nm}$ ) on CoNP-tipped CdSe@CdS nanorod “dumbbells" (nanorod L = 40 - $170 \mathrm{~nm}$ )when cast onto supporting substrates.[292] These structures were also captured by oxidation and the nanoscale Kirkendall effect to demonstrate the persistence of these structures in the dispersed state. The branched nature of these "dumbbell" CoNP-tipped nanorod assemblies was attributed to the fact that each colloidal monomer carried two functional A-B monomers in the form of two dipolar CoNP “tips.”[292]

Linear colloidal polymers were recently synthesized using CoNP-tipped nanorod colloidal monomers carrying a single, strongly dipolar, CoNP-tip per nanorod (Fig. 27).[293294] These "matchstick" CoNP-tipped nanorods (nanorod length $=60-150 \mathrm{~nm}$ ) spontaneously formed linear assemblies due to dipolar magnetic coupling of the cobalt domains $(\mathrm{D} \approx 20$ nm).[293] The attached CdSe@ CdS nanorods were carried as side chains with a high grafting density (ca. 90\%), in a fashion analogous to "grafting through" approaches in classical polymer science to prepare graft copolymers (Fig. 27a,b). The surface wetting and phase behavior of these "colloidal bottlebrush" structures were strongly dependent on the nanorod side chain length (which was tunable from $60 \mathrm{~nm}$ to $150 \mathrm{~nm}$ ), where longer nanorod side chains promoted more efficient surface wetting behavior on carbon-coated substrates (Fig. 27c,d). Colloidal bottlebrush side chain length was also correlated with a decreased tendency for lateral aggregation of colloidal polymer chains in thin films through site isolation of the colloidal polymer backbones due to steric interactions of nanorod side chains.[293]

INSERT FIGURE 27 AND FIGURE 27 CAPTION 
Synthetic routes to both segmented and statistical colloidal copolymers were also demonstrated for this dipolar colloidal bottlebrush system (Fig. 28).[293] These dipolar colloidal monomers were shown to be tightly bound within preformed chains due to strong magnetic interactions of constituent NPs (ca. $-30 \mathrm{k}_{B} \mathrm{~T}$ ), which enabled blending of dispersions of preformed colloidal polymers as a route to form segmented, or block, copolymers (Fig. 28a,b). The preparation of block-type colloidal copolymers was demonstrated by blending dipolar “bare” PS-CoNPs with “CoNP-tipped”CdSe@CdS nanorods possessing CoNPs of similar size and magnetization. Despite numerous processing variations, segmented colloidal copolymers were consistently obtained from blends of dipolar colloidal monomers. The formation of colloidal polymers with segmented compositions was attributed to the strong dipolar coupling between CoNP units which suppressed isotropic mixing of disparate colloids, where efficient mixing would be expected to afford statistical or random colloidal copolymers. These segmented colloidal polymers obtained from blending of preformed dipolar NP chains were thus obtained in mixtures with the parent homopolymers (Fig. 28c). Conversely, random colloidal copolymers of CoNP-tipped nanorods were prepared by blending "activated" nanorods of differing lengths prior to deposition of dipolar CoNP-tips. Since these "activated" CdSe $@ \mathrm{CdS}$ nanorods were not dipolar in nature, isotropic mixing "in solution" was obtained. Subsequent deposition of dipolar CoNP-tips resulted in the in situ formation of the desired statistical copolymer structure, and colloidal homopolymers were not observed from this approach.[293]

INSERT FIGURE 28 AND FIGURE 28 CAPTION 
The phase behavior of dipolar colloidal (co)polymers was also investigated by Pyun and coworkers by the blending of bare CoNPs with CoNP-tipped nanorods (Fig. 29b).[293] Segmented colloidal copolymers consisting of CoNPs and CoNP-tipped nanorods (10:1 ratio by number of particles) were cast into thin films on carbon coated TEM grids, and segments rich in bare CoNPs laterally associated into tightly bundled, , phase segregated domains of CoNP chains (Fig. 29a). Conversely, due to site isolation of the colloidal polymer backbone afforded by steric interactions of attached nanorod side chains, dipolar CoNP-tipped nanorods did not laterally selfassociate or associate with bare CoNP chains (Fig. 29a). This was the first example demonstrating the formation of a phase separated morphology from colloidal (co)polymers at this length scale, which highlighted how otherwise unexpected results can be obtained by applying concepts from classical polymer science to the self-assembly of inorganic colloids.

\section{INSERT FIGURE 29 AND FIGURE 29 CAPTION}

\section{Properties of colloidal polymers}

Although this field is still in the early stages of development, a number of structureproperty correlations have been investigated, pointing to the intriguing optical, electrical, electrochemical, and catalytic properties of colloidal polymers from inorganic NPs. Selfassembled structures of inorganic nanoparticles can exhibit synergistic properties which are not observed in the individual constituent particles,[189] as demonstrated by the early plasmonic sensor work of Mirkin and coworkers for the detection of oligonucleotides.[32, 295-297] However, the synthesis and functionalization of inorganic colloids for the formation of linear colloidal polymers is an emerging field which is only beginning to reveal material design rules 
and associated properties. The need for understanding structure/property correlations of colloidal polymers is, in part, responsible for driving synthetic efforts towards controlling fundamental polymer parameters (e.g., DP, composition, architecture) in these mescoscopic materials.

In a general sense, the characterization of electronic properties is complicated by the presence of stabilizing ligands used in the synthesis of inorganic colloids. These insulating organic ligands often comprise a significant mass percentage of the sample, which translates to a large volume fraction of organic material present with these colloidal polymers. For example, gold nanoparticles (density of $\mathrm{Au}, 19.3 \mathrm{~g} / \mathrm{cm}^{3}$ ) stabilized with citrate ligands (density of citric

acid, $1.665 \mathrm{~g} / \mathrm{cm}^{3}$ ) at $50 \%$ organic content by mass, exist as $92 \%$ organic ligand by volume in the composite material. This large volume of insulating material has been problematic for device manufacturing using inorganic NPs,[298] which will also apply to the incorporation of colloidal polymers into future devices.

\subsection{Properties correlated with DP, monomer spacing, and monomer orientation}

Control over structural features such as DP and monomer spacing in colloidal polymers was utilized by Kumacheva and coworkers for modulation of the optical (plasmonic) properties in AuNR colloidal polymer systems (Fig. 30).[189-193] Coupling of localized surface plasmon resonances (LSPRs) in AuNR chains resulted in the formation of periodic arrays of enhanced electric fields, or "hot-spots" in the spaces between AuNR colloidal monomers, which enabled study of the optical properties of these colloidal polymers by surface-enhanced Raman scattering (SERS).[190] Both the SERS intensity and extinction (absorption + scattering) of AuNR colloidal polymers were shown to increase with average aggregation number (DP) in the range of oligomeric chain lengths. This study further afforded a direct correlation between ensemble- 
averaged surface-enhanced Raman scattering and extinction properties (product of extinctions at $\lambda=785 \mathrm{~nm}$ and $821 \mathrm{~nm}$ ) of AuNRs in colloidal polymer chains (Fig. 30).[190] The authors suggested potential applications of these colloidal polymers for chemical and biological sensing, nanoelectronics, and plasmonic circuits.

\section{INSERT FIGURE 30 AND FIGURE 30 CAPTION}

One of the most unusual features of colloidal polymer structure relative to classical polymers is the effect of spacing and orientation of constituent monomers within self-assembled structures (Fig. 31). Interparticle spacing has been extensively studied for plasmonic colloidal polymers, where overlap of plasmonic wavefunctions is highly dependent on interparticle distance. Thus, interparticle distances have been directly correlated with the utility of these colloidal polymers in sensing applications - particularly for SERS measurements.[188] Relative orientation of NP units within colloidal polymers can also be tuned for a number of systems (e.g., Fig. 19), and nanoparticle orientation has been directly correlated with a decrease in SERS intensity for side-by-side assembly of AuNRs versus individual colloidal monomers (Fig. 31a,b).[192] This decrease in SERS intensity was attributed to destructive interference of the electric fields surrounding laterally associated AuNRs (Fig. 31c). These examples demonstrate how control of polymer parameters (DP, spacing, and orientation) directly correlate with the ensemble material properties for colloidal polymers.

INSERT FIGURE 31 AND FIGURE 31 CAPTION 


\subsection{Properties correlated with composition of colloidal polymers}

Methods to control fundamental polymer parameters are still being developed for dipoledirected colloidal polymerization approaches, such as the dipolar assembly of ferromagnetic CoNPs. However, methods to vary the composition of these colloidal polymers have been developed based on core@shell colloidal monomer morphologies and decoration of preformed colloidal polymer chains.

Pyun and coworkers investigated the composition-dependent electrochemical properties of $\mathrm{Co}_{\mathrm{x}} \mathrm{O}_{\mathrm{y}}$ nanowires functionalized with $\mathrm{Au}$ and $\mathrm{Pt}$ inclusions, where the potential formation of nano-Schottky type junctions between the metal oxide (p-type) and metallic nanoinclusions resulted in enhanced electrochemical properties of cobalt oxide nanowires (Fig. 32).[280, 283] Cyclic voltammetry of $\mathrm{Au}-\mathrm{Co}_{3} \mathrm{O}_{4}$ versus $\mathrm{Co}_{3} \mathrm{O}_{4}$ nanowire films revealed a two-fold increase of the (background corrected) Faradaic electrochemical activity associated with redox transitions at the active sites (i.e., $\mathrm{CoO}_{2}>\mathrm{CoOOH}>\mathrm{Co}_{3} \mathrm{O}_{4} ; \mathrm{Fig}$. 32).[280] The capacitive current was also enhanced, giving a specific capacitance per NP of $\mathrm{C}_{\mathrm{Au}-\mathrm{C} 0304}=1.21 \times 10^{-13} \mathrm{~F}$ and $\mathrm{C}_{\mathrm{C} 0304}=4.50 \mathrm{x}$ $10^{-14} \mathrm{~F}$. This was the first report detailing the electrochemical properties of a nanoscopic metal oxide obtained via the nanoscale Kirkendall effect, and Pt-decorated $\mathrm{Co}_{3} \mathrm{O}_{4}$ nanowires were later demonstrated to exhibit a sevenfold enhancement in specific capacitance over pristine $\mathrm{Co}_{3} \mathrm{O}_{4}$ nanowires.[283] Kotov and coworkers investigated the electrochemical properties of individual CdTe NWs with and without coaxial silica coating using conductive AFM measurements, and observed that silica coatings as thin as $10 \mathrm{~nm}$ were strongly insulating.[299] Manna and coworkers also investigated charge transport across metal-semiconductor interfaces using "all inorganic networks" of nanowelded AuNP-tipped CdSe nanorods using micropatterned $\mathrm{Au} / \mathrm{SiO}_{2}$ 
electrodes, and found that the voltage dependence could be described by a Schottky barrier lowering at a metal semiconductor contact under reverse bias.[300]

\section{INSERT FIGURE 32 AND FIGURE 32 CAPTION}

Control of colloidal polymer composition also enabled direct comparison of $\mathrm{Co}_{3} \mathrm{O}_{4} \mathrm{NWs}$, Au- $\mathrm{Co}_{3} \mathrm{O}_{4} \mathrm{NWs}$, and blends of AuNPs with $\mathrm{Co}_{3} \mathrm{O}_{4} \mathrm{NWs}$ for formulation into negative electrodes for Li-batteries (Fig. 33).[282] Powder samples annealed at $500{ }^{\circ} \mathrm{C}$ were formulated with PVDF binder and Super P conductive carbon into a negative electrode for Li-batteries, and the electrochemical activity of these electrodes was assessed against Li reactivity using galvanostatic measurements. The heterostructured $\mathrm{Au}-\mathrm{Co}_{3} \mathrm{O}_{4}$ electrode was found to exhibit lowered capacity (on a mass basis) relative to $\mathrm{Co}_{3} \mathrm{O}_{4}$ electrodes (Fig. 33, blue circles and red squares, respectively). This lower capacity was attributed to the presence of AuNP inclusions, which lowered the specific capacity of these electrodes on a weight basis without enhancing the electroactivity of the nanocomposite toward Li-insertion. Furthermore, the $\mathrm{Au}-\mathrm{Co}_{3} \mathrm{O}_{4}$ exhibited more rapid loss of charge capacity with cycling relative to the other systems, which was attributed to an observed decrease in dimensional stability relative to $\mathrm{Co}_{3} \mathrm{O}_{4} \mathrm{NWs}$. By comparison, electrodes from physical mixtures of AuNPs and $\mathrm{Co}_{3} \mathrm{O}_{4} \mathrm{NWs}$ possessed a lower initial charge capacity than $\mathrm{Au}-\mathrm{Co}_{3} \mathrm{O}_{4} \mathrm{NWs}$, but improved retention of capacity versus cycle number (Fig. 33, green triangles).[282] Related work by Yan and coworkers investigated the use of Sb NWs and $\mathrm{CoSb}_{3}$ NWs as anode materials for Li-batteries.[278] These examples demonstrated direct correlation of colloidal polymer composition with the electrochemical properties of a bulk material. 


\section{INSERT FIGURE 33 AND FIGURE 33 CAPTION}

\section{Conclusions and outlook}

This Review has presented a classification of 1-D nanoparticle assembly in a new paradigm based on established ideas from classical polymer science. This classification of colloidal polymers is fundamentally linked to the mechanism governing colloidal polymer formation to aid future readers in their own investigations of nanoparticle assembly. Although the ability to dictate structure-property relationships in colloidal polymers is still in its infancy, continued efforts to develop increased control of colloidal polymer structure are anticipated to afford useful, synergistic properties. While many NP systems have been demonstrated to form linear assemblies, methods to control colloidal polymer architecture and composition have only been demonstrated for a limited number of NP systems. There remains a need to develop synthetic methods to control basic structural polymer parameters such as, DP, interparticle spacing, regiochemistry, composition and architecture. Hence, we anticipate that the synthesis of colloidal polymers will remain an emerging field since the subtle effects of DP, architecture, and composition on NP assemblies will need to be understood to afford useful nanocomposite materials.

\subsection{Acknowledgements}

This work was funded by the Division of Chemical Science, Geosciences and Biosciences, Office of Basic Energy Sciences of the U.S. Department of Energy (DE-FG0302ER15753) (L.J.H., J.P.), the NSF (DMR-0645618 and DMR-130792 for L.J.H., J.P.), the World Class University Program through the National Research Foundation of Korea funded by 
the Ministry of Education, Science and Technology (R31-10013) are acknowledged for support of this work. K.C. acknowledges financial support from NRF through the National Creative Research Initiative Center for Intelligent Hybrids (2010-0018290).

\subsection{References}

[1] Murray CB, Kagan CR, Bawendi MG. Self-organization of CdSe nanocrystallites into three- dimensional quantum dot superlattices. Science 1995;270:1335-1338.

[2] Redl FX, Cho KS, Murray CB, O'Brien S. Three-dimensional binary superlattices of magnetic nanocrystals and semiconductor quantum dots. Nature 2003;423:968-971.

[3] Shevchenko EV, Talapin DV, Kotov NA, O'Brien S, Murray CB. Structural diversity in binary nanoparticle superlattices. Nature 2006;439:55-59.

[4] Manna L, Scher EC, Alivisatos AP. Synthesis of soluble and processable rod-, arrow-, teardrop-, and tetrapod-shaped CdSe nanocrystals. J Am Chem Soc 2000;122:1270012706.

[5] Peng XG, Manna L, Yang WD, Wickham J, Scher E, Kadavanich A, Alivisatos AP. Shape control of CdSe nanocrystals. Nature 2000;404:59-61.

[6] Peng ZA, Peng X. Mechanisms of the shape evolution of CdSe nanocrystals. J Am Chem Soc 2001;123:1389-1395.

[7] Peng X. Mechanisms for the shape-control and shape-evolution of colloidal semiconductor nanocrystals. Adv Mater 2003;15:459-463.

[8] Manna L, Milliron DJ, Meisel A, Scher EC, Alivisatos AP. Controlled growth of tetrapod- branched inorganic nanocrystals. Nat Mater 2003;2:382-385.

[9] Milliron DJ, Hughes SM, Cui Y, Manna L, Li J, Wang LW, Paul Alivisatos A. Colloidal nanocrystal heterostructures with linear and branched topology. Nature 2004;430:190-195.

[10] Gu H, Zheng R, Zhang X, Xu B. Facile one-pot synthesis of bifunctional heterodimers of nanoparticles: a conjugate of quantum dot and magnetic nanoparticles. J Am Chem Soc 2004;126:5664-5665. 
[11] Gu H, Yang Z, Gao J, Chang CK, Xu B. Heterodimers of nanoparticles: formation at a liquid-liquid interface and particle-specific surface modification by functional molecules. J Am Chem Soc 2005;127:34-35.

[12] Teranishi T, Inoue Y, Nakaya M, Oumi Y, Sano T. Nanoacorns: anisotropically phasesegregated CoPd sulfide nanoparticles. J Am Chem Soc 2004;126:9914-9915.

[13] Yanqiu L, Qiang Z, Nurmikko AV, Shouheng S. Enhanced magnetooptical response in dumbbell- like Ag-CoFe2O4 nanoparticle pairs. Nano Lett 2005;5:1689-1692.

[14] Yu H, Chen M, Rice PM, Wang SX, White RL, Sun S. Dumbbell-like bifunctional $\mathrm{Au}-\mathrm{Fe} 3 \mathrm{O} 4$ nanoparticles. Nano Lett 2005;5:379-382.

[15] Choi SH, Kim EG, Hyeon T. One-pot synthesis of copper-indium sulfide nanocrystal heterostructures with acorn, bottle, and larva shapes. J Am Chem Soc 2006;128:25202521.

[16] Buck MR, Schaak RE. Emerging strategies for the total synthesis of inorganic nanostructures. Angew Chem Int Ed 2013;52:6154-6178.

[17] Costi R, Saunders AE, Banin U. Colloidal hybrid nanostructures: a new type of functional materials. Angew Chem Int Ed 2010;49:4878-4897.

[18] Banin U, Ben-Shahar Y, Vinokurov K. Hybrid semiconductor-metal nanoparticles: from architecture to function. Chem Mater 2014;26:97-110.

[19] Wang Y, Chen G, Yang M, Silber G, Xing S, Tan LH, Wang F, Feng Y, Liu X, Li S, Chen H. A systems approach towards the stoichiometry-controlled hetero-assembly of nanoparticles. Nat Commun 2010;1:87/1-7.

[20] Chen G, Wang Y, Tan LH, Yang M, Tan LS, Chen Y, Chen H. High-purity separation of gold nanoparticle dimers and trimers. J Am Chem Soc 2009;131:4218-4219.

[21] Buck MR, Bondi JF, Schaak RE. A total-synthesis framework for the construction of high-order colloidal hybrid nanoparticles. Nat Chem 2012;4:37-44.

[22] Odian G. Principles of polymerization, 4th Ed. New York: John Wiley \& Sons Inc, 2004. 832 pp. 
[23] Rowan SJ, Cantrill SJ, Cousins GRL, Sanders JKM, Stoddart JF. Dynamic covalent chemistry. Angew Chem Int Ed 2002;41:898-952.

[24] Brienne MJ, Gabard J, Lehn JM, Stibor I. Macroscopic expression of molecular recognition: supramolecular liquid crystalline phases induced by association of complementary heterocyclic components. Chem Commun 1989 :1868-1870.

[25] Fouquey C, Lehn JM, Levelut AM. Molecular recognition directed self-assembly of supramolecular liquid crystalline polymers from complementary chiral components. Adv Mater 1990;2:254-257.

[26] Brunsveld L, Folmer BJB, Meijer EW, Sijbesma RP. Supramolecular polymers. Chem Rev 2001;101:4071-4097.

[27] Lehn JM. Dynamers: dynamic molecular and supramolecular polymers. Prog Polym Sci 2005;30:814-831.

[28] De Greef TFA, Smulders MMJ, Wolffs M, Schenning A, Sijbesma RP, Meijer EW. Supramolecular polymerization. Chem Rev 2009;109:5687-5754.

[29] Wang JC, Neogi P, Forciniti D. On one-dimensional self-assembly of surfactant-coated nanoparticles. J Chem Phys 2006;125:1947171-1947176.

[30] Sinyagin AY, Belov A, Tang Z, Kotov NA. Monte Carlo computer simulation of chain formation from nanoparticles. J Phys Chem B 2006;110:7500-7507.

[31] Zhang H, Wang DY. Controlling the growth of charged-nanoparticle chains through interparticle electrostatic repulsion. Angew Chem Int Ed 2008;47:3984-3987.

[32] Elghanian R, Storhoff JJ, Mucic RC, Letsinger RL, Mirkin CA. Selective colorimetric detection of polynucleotides based on the distance-dependent optical properties of gold nanoparticles. Science 1997;277:1078-1081.

[33] Caswell KK, Wilson JN, Bunz UHF, Murphy CJ. Preferential end-to-end assembly of gold nanorods by biotin-streptavidin connectors. J Am Chem Soc 2003;125:1391413915. 
[34] Zhang SZ, Kou XS, Yang Z, Shi QH, Stucky GD, Sun LD, Wang JF, Yan CH. Nanonecklaces assembled from gold rods, spheres, and bipyramids. Chem Commun $2007: 1816-1818$.

[35] Cho KS, Talapin DV, Gaschler W, Murray CB. Designing PbSe nanowires and nanorings through oriented attachment of nanoparticles. J Am Chem Soc 2005;127:7140-7147.

[36] Figuerola A, Franchini IR, Fiore A, Mastria R, Falqui A, Bertoni G, Bals S, Van Tendeloo G, Kudera S, Cingolani R, Manna L. End-to-end assembly of shape-controlled nanocrystals via a nanowelding approach mediated by gold domains. Adv Mater 2009;21:550-554.

[37] Tang ZY, Kotov NA, Giersig M. Spontaneous organization of single CdTe nanoparticles into luminescent nanowires. Science 2002;297:237-240.

[38] Tripp SL, Pusztay SV, Ribbe AE, Wei A. Self-assembly of cobalt nanoparticle rings. J Am Chem Soc 2002;124:7914-7915.

[39] Mîinea LA, Sessions LB, Ericson KD, Glueck DS, Grubbs RB. Phenylethynylstyrene-cobalt carbonyl block copolymer composites. Macromolecules 2004;37:8967-8972.

[40] Grubbs RB. Hybrid metal-polymer composites from functional block copolymers. J Polym Sci Part A Polym Chem 2005;43:4323-4336.

[41] Huber DL. Synthesis, properties, and applications of iron nanoparticles. Small 2005;1:482-501.

[42] Wei A. Calixarene-encapsulated nanoparticles: self-assembly into functional nanomaterials. Chem Commun 2006 :1581-1591.

[43] Grubbs RB. Roles of polymer ligands in nanoparticle stabilization. Polym Rev 2007;47:197-215.

[44] Benkoski JJ, Bowles SE, Jones RL, Douglas JF, Pyun J, Karim A. Self-assembly of polymer- coated ferromagnetic nanoparticles into mesoscopic polymer chains. J Polym Sci Part B Polym Phys 2008;46:2267-2277. 
[45] Sudeep PK, Emrick T. Pearls of wisdom: stringing nanoparticles and polymers into new assemblies and materials. ACS Nano 2009;3:2870-2875.

[46] Wei A, Tripp SL, Liu J, Kasama T, Dunin-Borkowski RE. Calixarene-stabilized cobalt nanoparticle rings: self-assembly and collective magnetic properties. Supramol Chem 2009;21:189-195.

[47] Dai Q, Lam M, Swanson S, Yu RHR, Milliron DJ, Topuria T, Jubert PO, Nelson A. Monodisperse cobalt ferrite nanomagnets with uniform silica coatings. Langmuir 2010;26:17546-17551.

[48] Dai Q, Nelson A. Magnetically-responsive self assembled composites. Chem Soc Rev 2010;39:4057-4066.

[49] Wei A, Kasama T, Dunin-Borkowski RE. Self-assembly and flux closure studies of magnetic nanoparticle rings. J Mater Chem 2011;21:16686-16693.

[50] Hill L, Pyun J. Colloidal polymers via dipolar assembly of magnetic nanoparticle monomers. ACS Appl Mater Interfaces 2014;6:6022-6032.

[51] Thomas JR. Preparation and magnetic properties of colloidal cobalt particles. J Appl Phys 1966;37:2914-2915.

[52] Hess PH, Parker PH, Jr. Polymers for stabilization of colloidal cobalt particles. J Appl Polym Sci 1966;10:1915-1927.

[53] Faivre D, Schueler D. Magnetotactic bacteria and magnetosomes. Chem Rev 2008;108:4875-4898.

[54] Xia YN, Yang PD, Sun YG, Wu YY, Mayers B, Gates B, Yin YD, Kim F, Yan YQ. One- dimensional nanostructures: synthesis, characterization, and applications. Adv Mater 2003;15:353-389.

[55] Zhang ZL, Horsch MA, Lamm MH, Glotzer SC. Tethered nano building blocks: toward a conceptual framework for nanoparticle self-assembly. Nano Lett 2003;3:1341-1346.

[56] Glotzer SC, Solomon MJ, Kotov NA. Self-assembly: from nanoscale to microscale colloids. AIChE J 2004;50:2978-2985. 
[57] Xu L, Ma W, Wang L, Xu C, Kuang H, Kotov NA. Nanoparticle assemblies: dimensional transformation of nanomaterials and scalability. Chem Soc Rev 2013;42:3114-3126.

[58] Kitching H, Shiers MJ, Kenyon AJ, Parkin IP. Self-assembly of metallic nanoparticles into one dimensional arrays. J Mater Chem A 2013;1:6985-6999.

[59] Fullam S, Cottell D, Rensmo H, Fitzmaurice D. Carbon nanotube templated selfassembly and thermal processing of gold nanowires. Adv Mater 2000;12:1430-1432.

[60] Hutchinson TO, Liu YP, Kiely C, Kiely CJ, Brust M. Templated gold nanowire selfassembly on carbon substrates. Adv Mater 2001;13:1800-1803.

[61] Gates B, Wu Y, Yin Y, Yang P, Xia Y. Single-crystalline nanowires of Ag2Se can be synthesized by templating against nanowires of trigonal Se. J Am Chem Soc 2001;123:11500-11501.

[62] Xia Y, Gates B, Yin Y. Building complex structures from monodisperse spherical colloids. Aust J Chem 2001;54:287-290.

[63] Yin Y, Xia Y. Self-assembly of monodispersed spherical colloids into complex aggregates with well-defined sizes, shapes, and structures. Adv Mater 2001;13:267-271.

[64] Yin Y, Lu Y, Gates B, Xia Y. Template-assisted self-assembly: a practical route to complex aggregates of monodispersed colloids with well-defined sizes, shapes, and structures. J Am Chem Soc 2001;123:8718-8729.

[65] Yin Y, Lu Y, Xia Y. Assembly of monodispersed spherical colloids into onedimensional aggregates characterized by well-controlled structures and lengths. J Mater Chem 2001;11:987-989.

[66] Yin Y, Lu Y, Xia Y. A self-assembly approach to the formation of asymmetric dimers from monodispersed spherical colloids. J Am Chem Soc 2001;123:771-772.

[67] Warner MG, Hutchison JE. Linear assemblies of nanoparticles electrostatically organized on DNA scaffolds. Nat Mater 2003;2:272-277. 
[68] Dujardin E, Peet C, Stubbs G, Culver JN, Mann S. Organization of metallic nanoparticles using tobacco mosaic virus templates. Nano Lett 2003;3:413-417.

[69] Correa-Duarte MA, Sobal N, Liz-Marzan LM, Giersig M. Linear assemblies of silicacoated gold nanoparticles using carbon nanotubes as templates. Adv Mater 2004; $16: 2179$.

[70] Zhang M, Drechsler M, Mueller AHE. Template-controlled synthesis of wire-like cadmium sulfide nanoparticle assemblies within core-shell cylindrical polymer brushes. Chem Mater 2004;16:537-543.

[71] Liu Y, Goebl J, Yin Y. Templated synthesis of nanostructured materials. Chem Soc Rev 2013;42:2610-2653.

[72] He J, Liu YJ, Babu T, Wei ZJ, Nie ZH. Self-assembly of inorganic nanoparticle vesicles and tubules driven by tethered linear block copolymers. J Am Chem Soc 2012;134:11342-11345.

[73] Richter J, Seidel R, Kirsch R, Mertig M, Pompe W, Plaschke J, Schackert HK. Nanoscale palladium metallization of DNA. Adv Mater 2000;12:507-510.

[74] Richter J. Metallization of DNA. Physica E 2003;16:157-173.

[75] Wang J, Xia H, Zhang Y, Lu H, Kamat R, Dobrynin AV, Cheng J, Lin Y. Nucleationcontrolled polymerization of nanoparticles into supramolecular structures. J Am Chem Soc 2013;135:11417-11420.

[76] Liu YD, Choi HJ. Recent progress in smart polymer composite particles in electric and magnetic fields. Polym Int 2013;62:147-151.

[77] Wang M, He L, Yin Y. Magnetic field guided colloidal assembly. Mater Today 2013;16:110-116.

[78] Lin Y, Skaff H, Böker A, Dinsmore AD, Emrick T, Russell TP. Ultrathin cross-linked nanoparticle membranes. J Am Chem Soc 2003;125:12690-12691.

[79] Lin Y, Skaff H, Emrick T, Dinsmore AD, Russell TP. Nanoparticle assembly and transport at liquid-liquid interfaces. Science 2003;299:226-229. 
[80] Glogowski E, Tangirala R, Russell TP, Emrick T. Functionalization of nanoparticles for dispersion in polymers and assembly in fluids. J Polym Sci Part A Polym Chem 2006;44:5076-5086.

[81] Pham JT, Lawrence J, Lee DY, Grason GM, Emrick T, Crosby AJ. Highly stretchable nanoparticle helices through geometric asymmetry and surface forces. Adv Mater 2013;25:6703-6708.

[82] Lee DY, Pham JT, Lawrence J, Lee CH, Parkos C, Emrick T, Crosby AJ. Macroscopic nanoparticle ribbons and fabrics. Adv Mater 2013;25:1248-1253.

[83] Park S, Lim JH, Chung SW, Mirkin CA. Self-assembly of mesoscopic metal-polymer amphiphiles. Science 2004;303:348-351.

[84] Wang JY, Wang Y, Sheiko Sergei S, Betts Douglas E, DeSimone Joseph M. Tuning multiphase amphiphilic rods to direct self-assembly. J Am Chem Soc 2012;134:58015806.

[85] Vriezema DM, Aragones MC, Elemans JAAW, Cornelissen JJLM, Rowan AE, Nolte RJM. Self-assembled nanoreactors. Chem Rev 2005;105:1445-1489.

[86] Balazs AC, Emrick T, Russell TP. Nanoparticle polymer composites: where two small worlds meet. Science 2006;314:1107-1110.

[87] Pang X, Zhao L, Akinc M, Kim JK, Lin Z. Novel amphiphilic multi-arm, star-like block copolymers as unimolecular micelles. Macromolecules 2011;44:3746-3752.

[88] Pang XC, Zhao L, Feng CW, Lin ZQ. Novel amphiphilic multiarm, starlike coil-rod diblock copolymers via a combination of click chemistry with living polymerization. Macromolecules 2011;44:7176-7183.

[89] Yuan J, Xu Y, Muller AHE. One-dimensional magnetic inorganic-organic hybrid nanomaterials. Chem Soc Rev 2011;40:640-655.

[90] Pang X, He Y, Jiang B, Iocozzia J, Zhao L, Guo H, Liu J, Akinc M, Bowler N, Tan X, Lin Z. Block copolymer/ferroelectric nanoparticle nanocomposites. Nanoscale 2013;5:8695-8702. 
[91] Pang X, Zhao L, Han W, Xin X, Lin Z. A general and robust strategy for the synthesis of nearly monodisperse colloidal nanocrystals. Nat Nanotechnol 2013;8:426-431.

[92] Pang X, Wan C, Wang M, Lin Z. Strictly biphasic soft and hard Janus structures: synthesis, properties, and applications. Angew Chem Int Ed 2014;53:5524-5538.

[93] Manna L, Scher EC, Alivisatos AP. Shape control of colloidal semiconductor nanocrystals. J Cluster Sci 2002;13:521-532.

[94] Puzder A, Williamson AJ, Zaitseva N, Galli G, Manna L, Alivisatos AP. The effect of organic ligand binding on the growth of $\mathrm{CdSe}$ nanoparticles probed by ab initio calculations. Nano Lett 2004;4:2361-2365.

[95] Jana NR, Gearheart L, Murphy CJ. Wet chemical synthesis of high aspect ratio cylindrical gold nanorods. J Phys Chem B 2001;105:4065-4067.

[96] Jana NR, Gearheart L, Murphy CJ. Wet chemical synthesis of silver nanorods and nanowires of controllable aspect ratio. Chem Commun 2001 :617-618.

[97] Johnson CJ, Dujardin E, Davis SA, Murphy CJ, Mann S. Growth and form of gold nanorods prepared by seed-mediated, surfactant-directed synthesis. J Mater Chem 2002;12:1765-1770.

[98] Gao J, Bender CM, Murphy CJ. Dependence of the gold nanorod aspect ratio on the nature of the directing surfactant in aqueous solution. Langmuir 2003;19:9065-9070.

[99] Park K, Koerner H, Vaia RA. Depletion-induced shape and size selection of gold nanoparticles. Nano Lett 2010;10:1433-1439.

[100] Nepal D, Drummy LF, Biswas S, Park K, Vaia RA. Large scale solution assembly of quantum dot-gold nanorod architectures with plasmon enhanced fluorescence. ACS Nano 2013;7:9064-9074.

[101] Biswas S, Duan J, Nepal D, Park K, Pachter R, Vaia RA. Plasmon-induced transparency in the visible region via self-assembled gold nanorod heterodimers. Nano Lett 2013;13:6287-6291. 
[102] Wadams RC, Fabris L, Vaia RA, Park K. Time-dependent susceptibility of the growth of gold nanorods to the addition of a cosurfactant. Chem Mater 2013;25:4772-4780.

[103] Gupta MK, König T, Near R, Nepal D, Drummy LF, Biswas S, Naik S, Vaia RA, ElSayed MA, Tsukruk VV. Surface assembly and plasmonic properties in strongly coupled segmented gold nanorods. Small 2013;9:2979-2990.

[104] Zhang Q, Yin Y. Beyond spheres: Murphy's silver nanorods and nanowires. Chem Commun 2013;49:215-217.

[105] Park K, Biswas S, Kanel S, Nepal D, Vaia RA. Engineering the optical properties of gold nanorods: independent tuning of surface plasmon energy, extinction coefficient, and scattering cross section. J Phys Chem C 2014;118:5918-5926.

[106] Kumar S, Nann T. Shape control of II-VI semiconductor nanomaterials. Small 2006;2:316-329.

[107] Lattuada M, Hatton TA. Synthesis, properties and applications of Janus nanoparticles. Nano Today 2011;6:286-308.

[108] Murphy CJ, Sau TK, Gole AM, Orendorff CJ, Gao J, Gou L, Hunyadi SE, Li T. Anisotropic metal nanoparticles: synthesis, assembly, and optical applications. J Phys Chem B 2005;109:13857-13870.

[109] Perez-Juste J, Pastoriza-Santos I, Liz-Marzan LM, Mulvaney P. Gold nanorods: synthesis, characterization and applications. Coord Chem Rev 2005;249:1870-1901.

[110] Grzelczak M, Perez-Juste J, Mulvaney P, Liz-Marzan LM. Shape control in gold nanoparticle synthesis. Chem Soc Rev 2008;37:1783-1791.

[111] Huang XH, Neretina S, El-Sayed MA. Gold nanorods: from synthesis and properties to biological and biomedical applications. Adv Mater 2009;21:4880-4910.

[112] Mokari T, Rothenberg E, Popov I, Costi R, Banin U. Selective growth of metal tips onto semiconductor quantum rods and tetrapods. Science 2004;304:1787-1790. 
[113] Mokari T, Sztrum CG, Salant A, Rabani E, Banin U. Formation of asymmetric onesided metal- tipped semiconductor nanocrystal dots and rods. Nat Mater 2005;4:855863.

[114] Salant A, Amitay-Sadovsky E, Banin U. Directed self-assembly of gold-tipped CdSe nanorods. J Am Chem Soc 2006;128:10006-10007.

[115] Klinkova A, Choueiri RM, Kumacheva E. Self-assembled plasmonic nanostructures. Chem Soc Rev 2014;43:3976-3991.

[116] Hauser EA, Lynn JE, Experiments in colloid chemistry. New York: McGraw-Hill Book Co, 1940. 178 pp.

[117] Turkevich J, Stevenson PC, Hillier J. The nucleation and growth processes in the synthesis of colloidal gold. Discuss Faraday Soc 1951;11:55-75.

[118] Frens G. Controlled nucleation for the regulation of the particle size in monodisperse gold suspensions. Nature Phys Sci 1973;241:20-22.

[119] Romo-Herrera JM, Alvarez-Puebla RA, Liz-Marzan LM. Controlled assembly of plasmonic colloidal nanoparticle clusters. Nanoscale 2011;3:1304-1315.

[120] Crespo P, Litran R, Rojas TC, Multigner M, de la Fuente JM, Sanchez-Lopez JC, Garcia MA, Hernando A, Penades S, Fernandez A. Permanent magnetism, magnetic anisotropy, and hysteresis of thiol-capped gold nanoparticles. Phys Rev Lett 2004;93:087204/1-4.

[121] Lin S, Li M, Dujardin E, Girard C, Mann S. One-dimensional plasmon coupling by facile self- assembly of gold nanoparticles into branched chain networks. Adv Mater 2005;17:2553.

[122] Li M, Johnson S, Guo HT, Dujardin E, Mann S. A generalized mechanism for ligandinduced dipolar assembly of plasmonic gold nanoparticle chain networks. Adv Funct Mater 2011;21:851-859.

[123] Gangula A, Chelli J, Bukka S, Poonthiyil V, Podila R, Kannan R, Rao AM. Thione-gold nanoparticles interactions: Vroman-like effect, self-assembly and sensing. J Mater Chem 2012;22:22866-22872. 
[124] Zhang H, Fung KH, Hartmann Jr, Chan CT, Wang D. Controlled chainlike agglomeration of charged gold nanoparticles via a deliberate interaction balance. J Phys Chem C 2008;112:16830-16839.

[125] Yang MX, Chen G, Zhao YF, Silber G, Wang Y, Xing SX, Han Y, Chen HY. Mechanistic investigation into the spontaneous linear assembly of gold nanospheres. Phys Chem Chem Phys 2010;12:11850-11860.

[126] Han XG, Goebl J, Lu ZD, Yin YD. Role of salt in the spontaneous assembly of charged gold nanoparticles in ethanol. Langmuir 2011;27:5282-5289.

[127] Enustun BV, Turkevich J. Coagulation of colloidal gold. J Am Chem Soc 1963;85:3317-3328.

[128] Shipway AN, Lahav M, Gabai R, Willner I. Investigations into the electrostatically induced aggregation of Au nanoparticles. Langmuir 2000;16:8789-8795.

[129] El-Sayed MA. Some interesting properties of metals confined in time and nanometer space of different shapes. Acc Chem Res 2001;34:257-264.

[130] Liao J, Zhang Y, Yu W, Xu L, Ge C, Liu J, Gu N. Linear aggregation of gold nanoparticles in ethanol. Colloids Surf A 2003;223:177-183.

[131] Liao JH, Chen KJ, Xu LN, Ge CW, Wang J, Huang L, Gu N. Self-assembly of lengthtunable gold nanoparticle chains in organic solvents. Appl Phys A 2003;76:541-543.

[132] Cho EC, Choi SW, Camargo PHC, Xia Y. Thiol-induced assembly of Au nanoparticles into chainlike structures and their fixing by encapsulation in silica shells or gelatin microspheres. Langmuir 2010;26:10005-10012.

[133] Mondain-Monval O, Leal-Calderon F, Phillip J, Bibette J. Depletion forces in the presence of electrostatic double layer repulsion. Phys Rev Lett 1995;75:3364-3367.

[134] Choi SW, Cheong IW, Kim JH, Xia Y. Preparation of uniform microspheres using a simple fluidic device and their crystallization into close-packed lattices. Small 2009;5:454-459. 
[135] Lazarides AA, Schatz GC. DNA-linked metal nanosphere materials: structural basis for the optical properties. J Phys Chem B 2000;104:460-467.

[136] Pecharroman C. Influence of the close sphere interaction on the surface plasmon resonance absorption peak. Phys Chem Chem Phys 2009;11:5922-5929.

[137] Choueiri RM, Klinkova A, Thérien-Aubin H, Rubinstein M, Kumacheva E. Structural transitions in nanoparticle assemblies governed by competing nanoscale forces. J Am Chem Soc 2013;135:10262-10265.

[138] Xia H, Su G, Wang D. Size-dependent electrostatic chain growth of pH-sensitive hairy nanoparticles. Angew Chem Int Ed 2013;52:3726-3730.

[139] Xia H, Bai S, Hartmann Jr, Wang D. Synthesis of monodisperse quasi-spherical gold nanoparticles in water via silver(I)-assisted citrate reduction. Langmuir 2010;26:35853589 .

[140] Bain CD, Whitesides GM. Molecular-level control over surface order in self-assembled monolayer films of thiols on gold. Science 1988;240:62-63.

[141] Nam JM, Thaxton CS, Mirkin CA. Nanoparticle-based bio-bar codes for the ultrasensitive detection of proteins. Science 2003;301:1884-1886.

[142] Rosi NL, Mirkin CA. Nanostructures in biodiagnostics. Chem Rev 2005;105:1547-1562.

[143] Stewart ME, Anderton CR, Thompson LB, Maria J, Gray SK, Rogers JA, Nuzzo RG. Nanostructured plasmonic sensors. Chem Rev 2008;108:494-521.

[144] Tan SJ, Campolongo MJ, Luo D, Cheng WL. Building plasmonic nanostructures with DNA. Nat Nanotechnol 2011;6:268-276.

[145] Jones MR, Osberg KD, Macfarlane RJ, Langille MR, Mirkin CA. Templated techniques for the synthesis and assembly of plasmonic nanostructures. Chem Rev 2011;111:37363827.

[146] Zhao Y, Xu LG, Liz-Marzan LM, Kuang H, Ma W, Asenjo-Garcia A, de Abajo FJG, Kotov NA, Wang LB, Xu CL. Alternating plasmonic nanoparticle heterochains made by 
polymerase chain reaction and their optical properties. J Phys Chem Lett 2013;4:641647.

[147] Chen W, Bian A, Agarwal A, Liu LQ, Shen HB, Wang LB, Xu CL, Kotov NA. Nanoparticle superstructures made by polymerase chain reaction: collective interactions of nanoparticles and a new principle for chiral materials. Nano Lett 2009;9:2153-2159.

[148] Zhao Y, Xu LG, Kuang H, Wang LB, Xu CL. Asymmetric and symmetric PCR of gold nanoparticles: a pathway to scaled-up self-assembly with tunable chirality. J Mater Chem 2012;22:5574-5580.

[149] Ma W, Yin HH, Xu LG, Wang LB, Kuang H, Xu CL. A PCR based magnetic assembled sensor for ultrasensitive DNA detection. Chem Commun 2013;49:53695371.

[150] Kuang H, Ma W, Xu LG, Wang LB, Xu CL. Nanoscale superstructures assembled by polymerase chain reaction (PCR): programmable construction, structural diversity, and emerging applications. Acc Chem Res 2013;46:2341-2354.

[151] Xu LG, Kuang H, Xu CL, Ma W, Wang LB, Kotov NA. Regiospecific plasmonic assemblies for in situ raman spectroscopy in live cells. J Am Chem Soc 2012;134:16991709.

[152] Xu LG, Hao CL, Yin HH, Liu LQ, Ma W, Wang LB, Kuang H, Xu CL. Plasmonic coresatellites nanostructures with high chirality and bioproperty. J Phys Chem Lett $2013 ; 4: 2379-2384$.

[153] DeVries GA, Brunnbauer M, Hu Y, Jackson AM, Long B, Neltner BT, Uzun O, Wunsch BH, Stellacci F. Divalent metal nanoparticles. Science 2007;315:358-361.

[154] Brust M, Walker M, Bethell D, Schiffrin DJ, Whyman R. Synthesis of thiol-derivatised gold nanoparticles in a two-phase liquid-liquid system. Chem Commun 1994 :801-802.

[155] Daniel MC, Astruc D. Gold nanoparticles: assembly, supramolecular chemistry, quantum-size- related properties, and applications toward biology, catalysis, and nanotechnology. Chem Rev 2004;104:293-346. 
[156] Sardar R, Funston AM, Mulvaney P, Murray RW. Gold nanoparticles: past, present, and future. Langmuir 2009;25:13840-13851.

[157] Boisselier E, Astruc D. Gold nanoparticles in nanomedicine: preparations, imaging, diagnostics, therapies and toxicity. Chem Soc Rev 2009;38:1759-1782.

[158] Jackson AM, Myerson JW, Stellacci F. Spontaneous assembly of subnanometre-ordered domains in the ligand shell of monolayer-protected nanoparticles. Nat Mater 2004;3:330-336.

[159] Cesbron Y, Shaw CP, Birchall JP, Free P, Lévy R. Stripy nanoparticles revisited. Small 2012;8:3714-3719.

[160] Yu M, Stellacci F. Response to "Stripy nanoparticles revisited". Small 2012;8:37203726.

[161] Nakata K, Hu Y, Uzun O, Bakr O, Stellacci F. Chains of superparamagnetic nanoparticles. Adv Mater 2008;20:4294-4299.

[162] Shibu Joseph ST, Ipe BI, Pramod P, Thomas KG. Gold nanorods to nanochains: mechanistic investigations on their longitudinal assembly using $\alpha, \omega$-alkanedithiols and interplasmon coupling. J Phys Chem B 2006;110:150-157.

[163] Thomas KG, Barazzouk S, Ipe BI, Joseph STS, Kamat PV. Uniaxial plasmon coupling through longitudinal self-assembly of gold nanorods. J Phys Chem B 2004;108:1306613068.

[164] Pramod P, Thomas KG. Plasmon coupling in dimers of Au nanorods. Adv Mater 2008;20:4300-4305.

[165] Sudeep PK, Joseph STS, Thomas KG. Selective detection of cysteine and glutathione using gold nanorods. J Am Chem Soc 2005;127:6516-6517.

[166] Zeiri O, Wang Y, Neyman A, Stellacci F, Weinstock IA. Ligand-shell-directed assembly and depolymerization of patchy nanoparticles. Angew Chem Int Ed 2013;52:968-972.

[167] Zhang Z, Glotzer SC. Self-assembly of patchy particles. Nano Lett 2004;4:1407-1413. 
[168] Sau TK, Murphy CJ. Self-assembly patterns formed upon solvent evaporation of aqueous cetyltrimethylammonium bromide-coated gold nanoparticles of various shapes. Langmuir 2005;21:2923-2929.

[169] Nie Z, Fava D, Kumacheva E, Zou S, Walker GC, Rubinstein M. Self-assembly of metal-polymer analogues of amphiphilic triblock copolymers. Nat Mater 2007;6:609614.

[170] Chen T, Yang M, Wang X, Tan LH, Chen H. Controlled assembly of eccentrically encapsulated gold nanoparticles. J Am Chem Soc 2008;130:11858-11859.

[171] Wang X, Li G, Chen T, Yang M, Zhang Z, Wu T, Chen H. Polymer-encapsulated goldnanoparticle dimers: facile preparation and catalytical application in guided growth of dimeric ZnO- nanowires. Nano Lett 2008;8:2643-2647.

[172] Tan LH, Xing SX, Chen T, Chen G, Huang X, Zhang H, Chen HY. Fabrication of polymer nanocavities with tailored openings. ACS Nano 2009;3:3469-3474.

[173] Xing S, Tan LH, Yang M, Pan M, Lv Y, Tang Q, Yang Y, Chen H. Highly controlled core/shell structures: tunable conductive polymer shells on gold nanoparticles and nanochains. J Mater Chem 2009;19:3286-3291.

[174] Xing SX, Feng YH, Tay YY, Chen T, Xu J, Pan M, He JT, Hng HH, Yan QY, Chen HY. Reducing the symmetry of bimetallic Au@Ag nanoparticles by exploiting eccentric polymer shells. J Am Chem Soc 2010;132:9537-9539.

[175] Xing S, He J, Liu X, Chen H. A symmetry-adapted shell transformation of core-shell nanoparticles for binary nanoassembly. Chem Commun 2011;47:12533-12535.

[176] Li W, Zhang P, Dai M, He J, Babu T, Xu YL, Deng R, Liang R, Lu MH, Nie Z, Zhu J. Ordering of gold nanorods in confined spaces by directed assembly. Macromolecules 2013;46:2241-2248.

[177] Wang J, Li W, Zhu J. Encapsulation of inorganic nanoparticles into block copolymer micellar aggregates: Strategies and precise localization of nanoparticles. Polymer 2014;55:1079-1096. 
[178] Kang Y, Erickson KJ, Taton TA. Plasmonic nanoparticle chains via a morphological, sphere-to- string transition. J Am Chem Soc 2005;127:13800-13801.

[179] Kang Y, Taton TA. Core/shell gold nanoparticles by self-assembly and crosslinking of micellar, block-copolymer shells. Angew Chem Int Ed 2005;44:409-412.

[180] Wang H, Chen L, Shen X, Zhu L, He J, Chen H. Unconventional chain-growth mode in the assembly of colloidal gold nanoparticles. Angew Chem Int Ed 2012;51:8021-8025.

[181] Park HS, Agarwal A, Kotov NA, Lavrentovich OD. Controllable side-by-side and endto-end assembly of Au nanorods by lyotropic chromonic materials. Langmuir 2008;24:13833-13837.

[182] Sanchez-Iglesias A, Grzelczak M, Perez-Juste J, Liz-Marzan LM. Binary self-assembly of gold nanowires with nanospheres and nanorods. Angew Chem Int Ed 2010;49:99859989.

[183] Fava D, Nie Z, Winnik MA, Kumacheva E. Evolution of self-assembled structures of polymer- terminated gold nanorods in selective solvents. Adv Mater 2008;20:43184322.

[184] Nie Z, Fava D, Rubinstein M, Kumacheva E. "Supramolecular" assembly of gold nanorods end- terminated with polymer "pom-poms": effect of pom-pom structure on the association modes. J Am Chem Soc 2008;130:3683-3689.

[185] Fava D, Winnik MA, Kumacheva E. Photothermally-triggered self-assembly of gold nanorods. Chem Commun 2009 :2571-2573.

[186] Liu K, Nie Z, Zhao N, Li W, Rubinstein M, Kumacheva E. Step-growth polymerization of inorganic nanoparticles. Science 2010;329:197-200.

[187] Liu K, Resetco C, Kumacheva E. Salt-mediated kinetics of the self-assembly of gold nanorods end-tethered with polymer ligands. Nanoscale 2012;4:6574-6580.

[188] Lukach A, Liu K, Therien-Aubin H, Kumacheva E. Controlling the degree of polymerization, bond lengths, and bond angles of plasmonic polymers. J Am Chem Soc 2012;134:18853-18859. 
[189] Nie Z, Petukhova A, Kumacheva E. Properties and emerging applications of selfassembled structures made from inorganic nanoparticles. Nat Nanotechnol 2010;5:1525.

[190] Lee A, Andrade GFS, Ahmed A, Souza ML, Coombs N, Tumarkin E, Liu K, Gordon R, Brolo AG, Kumacheva E. Probing dynamic generation of hot-spots in self-assembled chains of gold nanorods by surface-enhanced Raman scattering. J Am Chem Soc 2011;133:7563-7570.

[191] Eftekhari F, Lee A, Kumacheva E, Helmy AS. Examining metal nanoparticle surface chemistry using hollow-core, photonic-crystal, fiber-assisted SERS. Opt Lett 2012;37:680-682.

[192] Lee A, Ahmed A, dos Santos DP, Coombs N, Park JI, Gordon R, Brolo AG, Kumacheva E. Side- by-side assembly of gold nanorods reduces ensemble-averaged SERS intensity. J Phys Chem C 2012;116:5538-5545.

[193] Liu K, Ahmed A, Chung S, Sugikawa K, Wu G, Nie Z, Gordon R, Kumacheva E. In situ plasmonic counter for polymerization of chains of gold nanorods in solution. ACS Nano 2013;7:5901-5910.

[194] Klinkova A, Therien-Aubin H, Choueiri RM, Rubinstein M, Kumacheva E. Colloidal analogs of molecular chain stoppers. Proc Natl Acad Sci USA 2013;110:18775-18779.

[195] Liu K, Lukach A, Sugikawa K, Chung S, Vickery J, Therien-Aubin H, Yang B, Rubinstein M, Kumacheva E. Copolymerization of metal nanoparticles: a route to colloidal plasmonic copolymers. Angew Chem 2014;126:2648-2653.

[196] Zhao N, Vickery J, Guerin G, Park JI, Winnik MA, Kumacheva E. Self-assembly of single-tip metal-semiconductor nanorods in selective solvents. Angew Chem Int Ed 2011;50:4606-4610.

[197] Rycenga M, Cobley CM, Zeng J, Li WY, Moran CH, Zhang Q, Qin D, Xia YN. Controlling the synthesis and assembly of silver nanostructures for plasmonic applications. Chem Rev 2011;111:3669-3712. 
[198] Shiers MJ, Leech R, Carmalt CJ, Parkin IP, Kenyon AJ. Self-assembled ultra-high aspect ratio silver nanochains. Adv Mater 2012;24:5227-5235.

[199] Smetana AB, Klabunde KJ, Sorensen CM. Synthesis of spherical silver nanoparticles by digestive ripening, stabilization with various agents, and their 3-D and 2-D superlattice formation. J Colloid Interface Sci 2005;284:521-526.

[200] Gao B, Arya G, Tao AR. Self-orienting nanocubes for the assembly of plasmonic nanojunctions. Nat Nanotechnol 2012;7:433-437.

[201] Asakura S, Oosawa F. Interaction between two bodies immersed in a solution of macromolecules. J Chem Phys 1954;22:1255-1256.

[202] Pryamtisyn V, Ganesan V, Panagiotopoulos AZ, Liu H, Kumar SK. Modeling the anisotropic self-assembly of spherical polymer-grafted nanoparticles. J Phys Chem 2009;131:221102/1-4.

[203] Li L, Miesch C, Sudeep PK, Balazs AC, Emrick T, Russell TP, Hayward RC. Kinetically trapped co-continuous polymer morphologies through intraphase gelation of nanoparticles. Nano Lett 2011;11:1997-2003.

[204] Penn RL, Banfield JF. Oriented attachment and growth, twinning, polytypism, and formation of metastable phases: insights from nanocrystalline TiO2. Am Mineral 1998;83:1077-1082.

[205] Penn RL, Banfield JF. Imperfect oriented attachment: dislocation generation in defectfree nanocrystals. Science 1998;281:969-971.

[206] Penn RL, Banfield JF. Morphology development and crystal growth in nanocrystalline aggregates under hydrothermal conditions: insights from titania. Geochim Cosmochim Acta 1999;63:1549-1557.

[207] Banfield JF, Welch SA, Zhang H, Ebert TT, Penn RL. Aggregation-based crystal growth and microstructure development in natural iron oxyhydroxide biomineralization products. Science 2000;289:751-754.

[208] Xue X, Penn RL, Leite ER, Huang F, Lin Z. Crystal growth by oriented attachment: kinetic models and control factors. CrystEngComm 2014;16:1419-1429. 
[209] Zhang J, Huang F, Lin Z. Progress of nanocrystalline growth kinetics based on oriented attachment. Nanoscale 2010;2:18-34.

[210] Ribeiro C, Lee EJH, Longo E, Leite ER. Oriented attachment mechanism in anisotropic nanocrystals: a "polymerization" approach. Chemphyschem 2006;7:664-670.

[211] Lu L, Kobayashi A, Kikkawa Y, Tawa K, Ozaki Y. Oriented attachment-based assembly of dendritic silver nanostructures at room temperature. J Phys Chem B 2006;110:23234-23241.

[212] Wen X, Xie YT, Mak MWC, Cheung KY, Li XY, Renneberg R, Yang S. Dendritic nanostructures of silver: facile synthesis, structural characterizations, and sensing applications. Langmuir 2006;22:4836-4842.

[213] Halder A, Ravishankar N. Ultrafine single-crystalline gold nanowire arrays by oriented attachment. Adv Mater 2007;19:1854-1858.

[214] Wang ZL. Transmission electron microscopy of shape-controlled nanocrystals and their assemblies. J Phys Chem B 2000;104:1153-1175.

[215] Polleux J, Pinna N, Antonietti M, Niederberger M. Ligand-directed assembly of preformed titania nanocrystals into highly anisotropic nanostructures. Adv Mater 2004;16:436-439.

[216] Pacholski C, Kornowski A, Weller H. Self-assembly of ZnO: from nanodots to nanorods. Angew Chem Int Ed 2002;41:1188-1191.

[217] Pradhan N, Xu H, Peng X. Colloidal CdSe quantum wires by oriented attachment. Nano Lett 2006;6:720-724.

[218] Tang Z, Kotov NA. One-dimensional assemblies of nanoparticles: preparation, properties, and promise. Adv Mater 2005;17:951-962.

[219] Liu B, Zeng HC. Semiconductor rings fabricated by self-assembly of nanocrystals. J Am Chem Soc 2005; 127:18262-18268.

[220] Li Z, Hu Y, Sun Y. Promoting photocatalytic multiple-electron reduction in aerobic solutions using Au-tipped CdSe nanorod clusters. Chem Commun 2014;50:1411-1413. 
[221] Benkoski JJ, Jones RL, Douglas JF, Karim A. Photocurable oil/water interfaces as a universal platform for 2-D self-assembly. Langmuir 2007;23:3530-3537.

[222] Tavares JM, Weis JJ, Telo dGMM. Quasi-two-dimensional dipolar fluid at low densities: Monte Carlo simulations and theory. Phys Rev E 2002;65:061201.

[223] Vandewalle N, Ausloos M. Magnetic diffusion-limited aggregation. Phys Rev E 1995;51:597-603.

[224] Rogach AL, Katsikas L, Kornowski A, Su D, Eychmüller A, Weller H. Synthesis and characterization of thiol-stabilized CdTe nanocrystals. Ber Bunsenges Phys Chem 1996;100:1772-1778.

[225] Rogach AL, Nagesha D, Ostrander JW, Giersig M, Kotov NA. "Raisin bun"-type composite spheres of silica and semiconductor nanocrystals. Chem Mater 2000;12:2676-2685.

[226] Sinyagin A, Belov A, Kotov N. Monte Carlo simulation of linear aggregate formation from CdTe nanoparticles. Modelling Simul Mater Sci Eng 2005;13:389-399.

[227] Zhang Z, Tang Z, Kotov NA, Glotzer SC. Simulations and analysis of self-assembly of CdTe nanoparticles into wires and sheets. Nano Lett 2007;7:1670-1675.

[228] Tang Z, Zhang Z, Wang Y, Glotzer SC, Kotov NA. Self-assembly of CdTe nanocrystals into free- floating sheets. Science 2006;314:274-278.

[229] Tang Z, Wang Y, Sun K, Kotov NA. Spontaneous transformation of stabilizer-depleted binary semiconductor nanoparticles into selenium and tellurium nanowires. Adv Mater 2005; 17:358-363.

[230] Lilly GD, Lee J, Sun K, Tang Z, Kim KS, Kotov NA. Media effect on CdTe nanowire growth: mechanism of self-assembly, Ostwald ripening, and control of NW geometry. J Phys Chem C 2008;112:370-377.

[231] Tang Z, Podsiadlo P, Shim BS, Lee J, Kotov NA. The effect of stabilizer density on transformation of CdTe nanoparticles induced by Ag cations. Adv Funct Mater 2008;18:3801-3808. 
[232] Wang Y, Tang Z, Liang X, Liz-Marzán LM, Kotov NA. SiO2-coated CdTe nanowires: bristled nano centipedes. Nano Lett 2004;4:225-231.

[233] Sheiko SS, Sumerlin BS, Matyjaszewski K. Cylindrical molecular brushes: synthesis, characterization, and properties. Prog Polym Sci 2008;33:759-785.

[234] Tang Z, Ozturk B, Wang Y, Kotov NA. Simple preparation strategy and onedimensional energy transfer in CdTe nanoparticle chains. J Phys Chem B 2004;108:6927-6931.

[235] Ma G, Zhou Y, Li X, Sun K, Liu S, Hu J, Kotov NA. Self-assembly of copper sulfide nanoparticles into nanoribbons with continuous crystallinity. ACS Nano 2013;7:90109018.

[236] Srivastava S, Santos A, Critchley K, Kim KS, Podsiadlo P, Sun K, Lee J, Xu CL, Lilly GD, Glotzer SC, Kotov NA. Light-controlled self-assembly of semiconductor nanoparticles into twisted ribbons. Science 2010;327:1355-1359.

[237] Yang M, Sun K, Kotov NA. Formation and assembly-disassembly processes of ZnO hexagonal pyramids driven by dipolar and excluded volume interactions. J Am Chem Soc 2010;132:1860-1872.

[238] Butter K, Bomans PHH, Frederik PM, Vroege GJ, Philipse AP. Direct observation of dipolar chains in iron ferrofluids by cryogenic electron microscopy. Nat Mater 2003;2:88-91.

[239] Klokkenburg M, Vonk C, Claesson EM, Meeldijk JD, Erne BH, Philipse AP. Direct imaging of zero-field dipolar structures in colloidal dispersions of synthetic magnetite. J Am Chem Soc 2004;126:16706-16707.

[240] Pyun J. Nanocomposite materials from functional polymers and magnetic colloids. Polym Rev 2007;47:231-263.

[241] Motornov M, Malynych SZ, Pippalla DS, Zdyrko B, Royter H, Roiter Y, Kahabka M, Tokarev A, Tokarev I, Zhulina E, Kornev KG, Luzinov I, Minko S. Field-directed selfassembly with locking nanoparticles. Nano Lett 2012;12:3814-3820. 
[242] Singamaneni S, Bliznyuk VN, Binek C, Tsymbal EY. Magnetic nanoparticles: recent advances in synthesis, self-assembly and applications. J Mater Chem 2011;21:1681916845.

[243] Griffiths CH, O'Horo MP, Smith TW. The structure, magnetic characterization and oxidation of colloidal iron dispersions. J Appl Phys 1979;50:7108-7115.

[244] Smith TW, Wychick D. Colloidal iron dispersions prepared via the polymer-catalyzed decomposition of iron pentacarbonyl. J Phys Chem 1980;84:1621-1629.

[245] Platonova OA, Bronstein LM, Solodovnikov SP, Yanovskaya IM, Obolonkova ES, Valetsky PM, Wenz E, Antonietti M. Cobalt nanoparticles in block copolymer micelles: preparation and properties. Colloid Polym Sci 1997;275:426-431.

[246] Burke NAD, Stover HDH, Dawson FR. Magnetic nanocomposites: preparation and characterization of polymer-coated iron nanoparticles. Chem Mater 2002;14:4752-4761.

[247] Diana FS, Lee SH, Petroff PM, Kramer EJ. Fabrication of hcp-Co nanocrystals via rapid pyrolysis in inverse PS-b-PVP micelles and thermal annealing. Nano Lett 2003;3:891895.

[248] Zhou Z, Liu G, Han D. Coating and structural locking of dipolar chains of cobalt nanoparticles. ACS Nano 2009;3:165-172.

[249] Dinega DP, Bawendi MG. A solution-phase chemical approach to a new crystal structure of cobalt. Angew Chem Int Ed 1999;38:1788-1791.

[250] Puntes VF, Krishnan KM, Alivisatos AP. Colloidal nanocrystal shape and size control: the case of cobalt. Science 2001;291:2115-2117.

[251] Puntes VF, Zanchet D, Erdonmez CK, Alivisatos AP. Synthesis of hcp-Co nanodisks. J Am Chem Soc 2002;124:12874-12880.

[252] Van Workum K, Douglas JF. Equilibrium polymerization in the Stockmayer fluid as a model of supermolecular self-organization. Phys Rev E 2005;71:031502.

[253] Tripp SL, Dunin-Borkowski RE, Wei A. Flux closure in self-assembled cobalt nanoparticle rings. Angew Chem Int Ed 2003;42:5591-5593. 
[254] Kasama T, Dunin-Borkowski RE, Scheinfein MR, Tripp SL, Liu J, Wei A. Reversal of flux closure states in cobalt nanoparticle rings with coaxial magnetic pulses. Adv Mater 2008;20:4248-4252.

[255] Hu MJ, Lu Y, Zhang S, Guo SR, Lin B, Zhang M, Yu SH. High yield synthesis of bracelet-like hydrophilic Ni-Co magnetic alloy flux-closure nanorings. J Am Chem Soc 2008;130:11606-11607.

[256] Ahmed W, Laarman RPB, Hellenthal C, Kooij ES, van Silfhout A, Poelsema B. Dipole directed ring assembly of Ni-coated Au-nanorods. Chem Commun 2010;46:6711-6713.

[257] Wang H, Chen QW, Sun YB, Wang MS, Sun LX, Yan WS. Synthesis of necklace-like magnetic nanorings. Langmuir 2010;26:5957-5962.

[258] Zhou S, Chen Q. Synthesis and characterization of bracelet-like magnetic nanorings consisting of Ag-Fe3O4 bi-component nanoparticles. Dalton Trans 2011;40:8622-8629.

[259] Ding WF, Li Z, Zhou H, Zhao B, Wan Jg, Song F, Wang GH. Tunable formation of ferromagnetic nanoparticle rings: experiments and Monte Carlo simulations. J Phys Chem C 2012;116:10805-10813.

[260] Korth BD, Keng P, Shim I, Bowles SE, Tang C, Kowalewski T, Nebesny KW, Pyun J. Polymer- coated ferromagnetic colloids from well-defined macromolecular surfactants and assembly into nanoparticle chains. J Am Chem Soc 2006;128:6562-6563.

[261] Keng PY, Shim I, Korth BD, Douglas JF, Pyun J. Synthesis and self-assembly of polymer-coated ferromagnetic nanoparticles. ACS Nano 2007;1:279-292.

[262] Bowles SE, Wu W, Kowalewski T, Schalnat MC, Davis RJ, Pemberton JE, Shim I, Korth BD, Pyun J. Magnetic assembly and pyrolysis of functional ferromagnetic colloids into one-dimensional carbon nanostructures. J Am Chem Soc 2007;129:86948695.

[263] Korth BD, Keng PY, Shim I, Tang C, Kowalewski T, Pyun J. Synthesis, assembly, and functionalization of polymer-coated ferromagnetic nanoparticles. ACS Symp Ser 2008;996:272-285. 
[264] Bull MM, Chung WJ, Anderson SR, Kim Sj, Shim IB, Paik Hj, Pyun J. Synthesis of ferromagnetic polymer coated nanoparticles on multi-gram scale with tunable particle size. J Mater Chem 2010;20:6023-6025.

[265] Benkoski JJ, Bowles SE, Korth BD, Jones RL, Douglas JF, Karim A, Pyun J. Field induced formation of mesoscopic polymer chains from functional ferromagnetic colloids. J Am Chem Soc 2007;129:6291-6297.

[266] Benkoski JJ, Breidenich JL, Uy OM, Hayes AT, Deacon RM, Land HB, Spicer JM, Keng PY, Pyun J. Dipolar organization and magnetic actuation of flagella-like nanoparticle assemblies. J Mater Chem 2011;21:7314-7325.

[267] Benkoski JJ, Deacon RM, Land HB, Baird LM, Breidenich JL, Srinivasan R, Clatterbaugh GV, Keng PY, Pyun J. Dipolar Assembly of Ferromagnetic Nanoparticles into Magnetically Driven Artificial Cilia. Soft Matter 2010;6:602-609.

[268] Yin Y, Rioux RM, Erdonmez CK, Hughes S, Somorjai GA, Alivisatos AP. Formation of hollow nanocrystals through the nanoscale Kirkendall effect. Science 2004;304:711714.

[269] Yin Y, Alivisatos AP. Colloidal nanocrystal synthesis and the organic-inorganic interface. Nature 2005;437:664-670.

[270] Yin Y, Erdonmez CK, Cabot A, Hughes S, Alivisatos AP. Colloidal synthesis of hollow cobalt sulfide nanocrystals. Adv Funct Mater 2006;16:1389-1399.

[271] Wang W, Dahl M, Yin Y. Hollow nanocrystals through the nanoscale Kirkendall effect. Chem Mater 2013;25:1179-1189.

[272] Gao J, Zhang B, Zhang X, Xu B. Magnetic-dipolar-interaction-induced self-assembly affords wires of hollow nanocrystals of cobalt selenide. Angew Chem Int Ed 2006; $45: 1220-1223$.

[273] Sun Y, Xia Y. Alloying and dealloying processes involved in the preparation of metal nanoshells through a galvanic replacement reaction. Nano Lett 2003;3:1569-1572.

[274] Moon GD, Ko S, Min Y, Zeng J, Xia Y, Jeong U. Chemical transformations of nanostructured materials. Nano Today 2011;6:186-203. 
[275] Zhang H, Jin M, Xia Y. Noble-metal nanocrystals with concave surfaces: synthesis and applications. Angew Chem Int Ed 2012;51:7656-7673.

[276] Xia X, Wang Y, Ruditskiy A, Xia Y. 25th anniversary article: galvanic replacement: a simple and versatile route to hollow nanostructures with tunable and well-controlled properties. Adv Mater 2013;25:6313-6333.

[277] Zeng J, Huang J, Lu W, Wang X, Wang B, Zhang S, Hou J. Necklace-like noble-metal hollow nanoparticle chains: synthesis and tunable optical properties. Adv Mater 2007;19:2172-2176.

[278] Zhu J, Sun T, Chen J, Shi W, Zhang X, Lou X, Mhaisalkar S, Hng HH, Boey F, Ma J, Yan Q. Controlled synthesis of Sb nanostructures and their conversion to CoSb3 nanoparticle chains for Li-ion battery electrodes. Chem Mater 2010;22:5333-5339.

[279] Keng PY, Kim BY, Shim IB, Sahoo R, Veneman PE, Armstrong NR, Yoo H, Pemberton JE, Bull MM, Griebel JJ, Ratcliff EL, Nebesny KG, Pyun J. Colloidal polymerization of polymer-coated ferromagnetic nanoparticles into cobalt oxide nanowires. ACS Nano 2009;3:3143-3157.

[280] Kim BY, Shim IB, Araci ZO, Saavedra SS, Monti OLA, Armstrong NR, Sahoo R, Srivastava DN, Pyun J. Synthesis and colloidal polymerization of ferromagnetic Au-Co nanoparticles into Au- Co3O4 nanowires. J Am Chem Soc 2010;132:3234-3235.

[281] Kim BY, Shim IB, Monti OLA, Pyun J. Magnetic self-assembly of gold nanoparticle chains using dipolar core-shell colloids. Chem Commun 2011;47:890-892.

[282] Kim BY, Yu SH, Kim HS, Lee DC, Shim IB, Derosa SE, Sung YE, Pyun J. Morphological conversion of dipolar core-shell Au-Co nanoparticles into beaded $\mathrm{Au}-$ Co3O4 nanowires. J Mater Chem 2011;21:14163-14166.

[283] Keng PY, Bull MM, Shim IB, Nebesny KG, Armstrong NR, Sung Y, Char K, Pyun J. Colloidal polymerization of polymer-coated ferromagnetic cobalt nanoparticles into PtCo3O4 nanowires. Chem Mater 2011;23:1120-1129.

[284] Buonsanti R, Grillo V, Carlino E, Giannini C, Curri ML, Innocenti C, Sangregorio C, Achterhold K, Parak FG, Agostiano A, Cozzoli PD. Seeded growth of asymmetric 
binary nanocrystals made of a semiconductor $\mathrm{TiO}_{2}$ rodlike section and a magnetic gamma-Fe2O3 spherical domain. J Am Chem Soc 2006;128:16953-16970.

[285] Casavola M, Grillo V, Carlino E, Giannini C, Gozzo F, Pinel EF, Garcia MA, Manna L, Cingolani R, Cozzoli PD. Topologically controlled growth of magnetic-metalfunctionalized semiconductor oxide nanorods. Nano Lett 2007;7:1386-1395.

[286] Zanella M, Falqui A, Kudera S, Manna L, Casula MF, Parak WJ. Growth of colloidal nanoparticles of group II-VI and IV-VI semiconductors on top of magnetic ironplatinum nanocrystals. J Mater Chem 2008;18:4311-4317.

[287] Buonsanti R, Snoeck E, Giannini C, Gozzo F, Garcia-Hernandez M, Garcia MA, Cingolani R, Cozzoli PD. Colloidal semiconductor/magnetic heterostructures based on iron-oxide-functionalized brookite $\mathrm{TiO}_{2}$ nanorods. Phys Chem Chem Phys 2009;11:3680-3691.

[288] Casavola M, Falqui A, Garcia MA, Garcia-Hernandez M, Giannini C, Cingolani R, Cozzoli PD. Exchange-coupled bimagnetic cobalt/iron oxide branched nanocrystal heterostructures. Nano Lett 2009;9:366-376.

[289] Deka S, Falqui A, Bertoni G, Sangregorio C, Poneti G, Morello G, De Giorgi M, Giannini C, Cingolani R, Manna L, Cozzoli PD. Fluorescent asymmetrically cobalttipped CdSe@CdS core@shell nanorod heterostructures exhibiting room-temperature ferromagnetic behavior. J Am Chem Soc 2009;131:12817-12828.

[290] Maynadie J, Salant A, Falqui A, Respaud M, Shaviv E, Banin U, Soulantica K, Chaudret B. Cobalt growth on the tips of CdSe nanorods. Angew Chem Int Ed 2009;48:18141817.

[291] Buonsanti R, Grillo V, Carlino E, Giannini C, Gozzo F, Garcia-Hernandez M, Garcia MA, Cingolani R, Cozzoli PD. Architectural control of seeded-grown magneticsemicondutor iron oxide- $\mathrm{TiO}(2)$ nanorod heterostructures: the role of seeds in topology selection. J Am Chem Soc 2010;132:2437-2464.

[292] Hill LJ, Bull MM, Sung Y, Simmonds AG, Dirlam PT, Richey NE, DeRosa SE, Shim IB, Guin D, Costanzo PJ, Pinna N, Willinger MG, Vogel W, Char K, Pyun J. Directing 
the deposition of ferromagnetic cobalt onto Pt-tipped CdSe@CdS nanorods: synthetic and mechanistic insights. ACS Nano 2012;6:8632-8645.

[293] Hill LJ, Richey NE, Sung Y, Dirlam PT, Lavoie-Higgins E, Shim IB, Pinna N, Willinger MG, Vogel W, Benkoski JJ, Char K, Pyun J. Colloidal polymers from dipolar assembly of cobalt-tipped CdSe@CdS nanorods. ACS Nano 2014;8:3272-3284.

[294] Hill LJ, Richey NE, Sung Y, Dirlam PT, Griebel JJ, Shim IB, Pinna N, Willinger MG, Vogel W, Char K, Pyun J. Synthesis of ferromagnetic cobalt nanoparticle tipped CdSe@CdS nanorods: critical role of Pt-activation. CrystEngComm 2014;DOI: 10.1039/c1034ce00680a.

[295] Mirkin CA, Letsinger RL, Mucic RC, Storhoff JJ. A DNA-based method for rationally assembling nanoparticles into macroscopic materials. Nature 1996;382:607-609.

[296] Alivisatos AP, Johnsson KP, Peng X, Wilson TE, Loweth CJ, Bruchez Jr MP, Schultz PG. Organization of 'nanocrystal molecules' using DNA. Nature 1996;382:609-611.

[297] Storhoff JJ, Mucic RC, Mirkin CA. Strategies for organizing nanoparticles into aggregate structures and functional materials. J Cluster Sci 1997;8:179-216.

[298] Kim JY, Kotov NA. Charge transport dilemma of solution-processed nanomaterials. Chem Mater 2014;26:134-152.

[299] Liang X, Tan S, Tang Z, Kotov NA. Investigation of transversal conductance in semiconductor CdTe nanowires with and without a coaxial silica shell. Langmuir 2004;20:1016-1020.

[300] Lavieville R, Zhang Y, Casu A, Genovese A, Manna L, Di Fabrizio E, Krahne R. Charge transport in nanoscale all-inorganic networks of semiconductor nanorods linked by metal domains. ACS Nano 2012;6:2940-2947. 


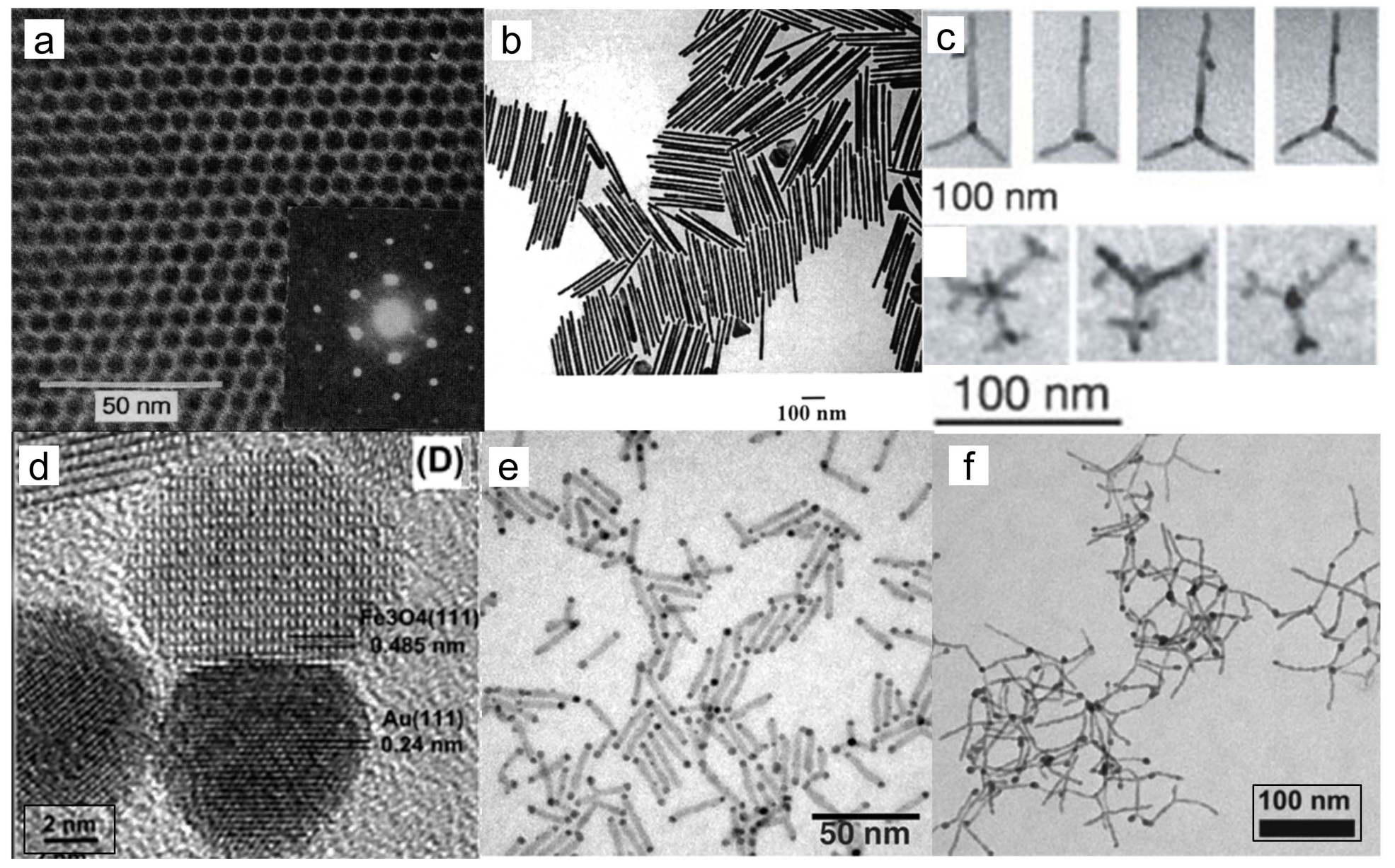


Figure 2

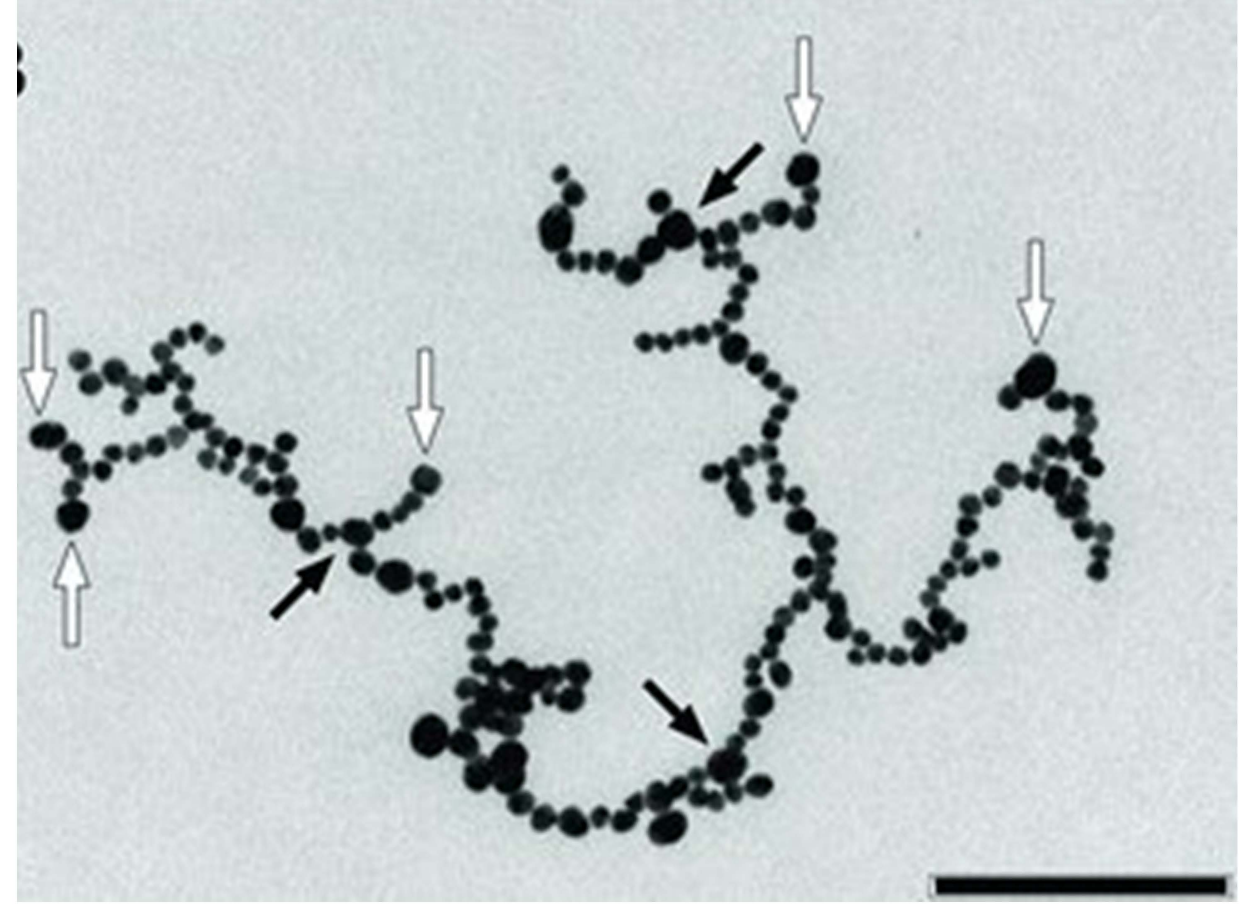


Figure 4

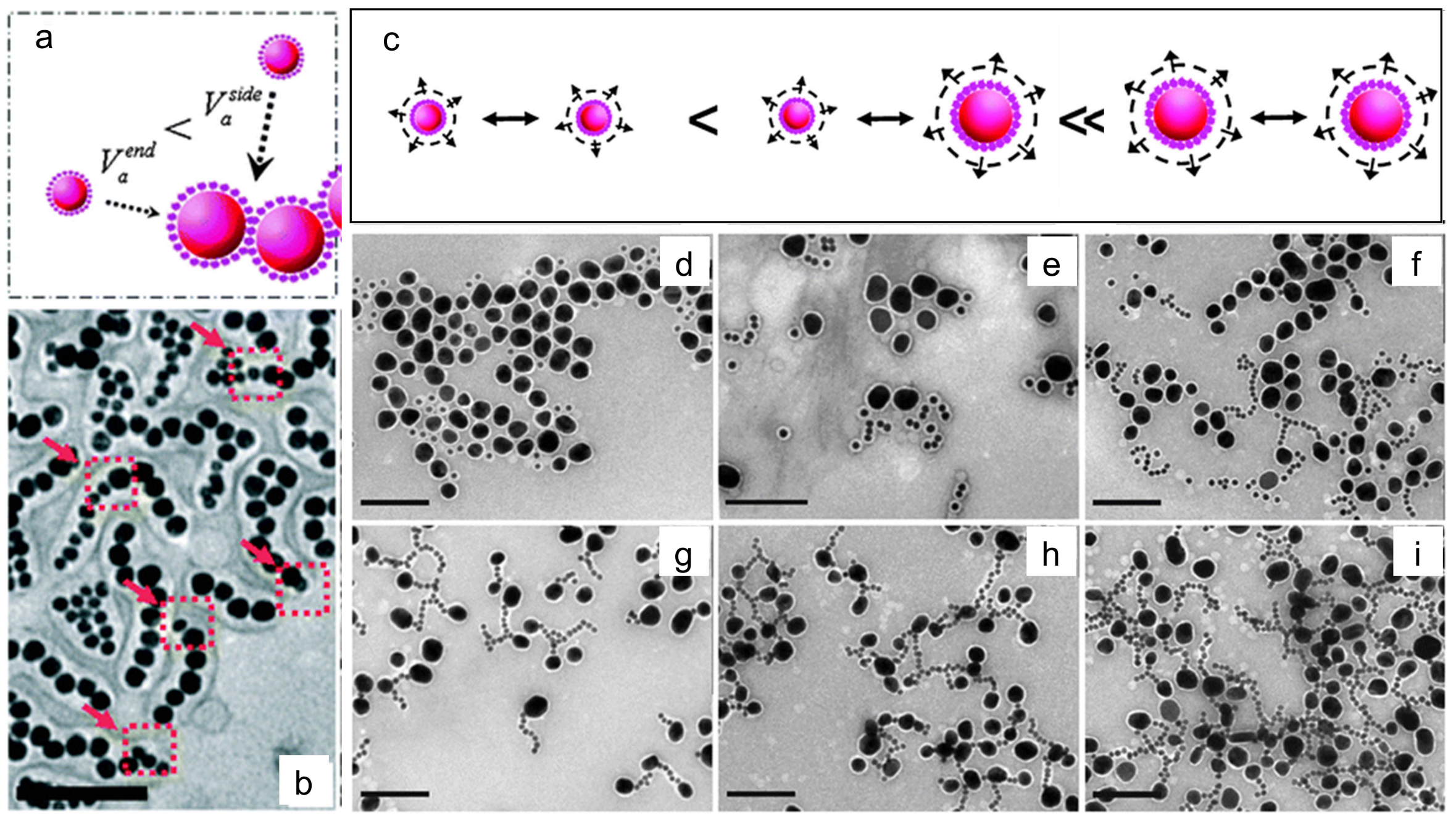


Figure 5

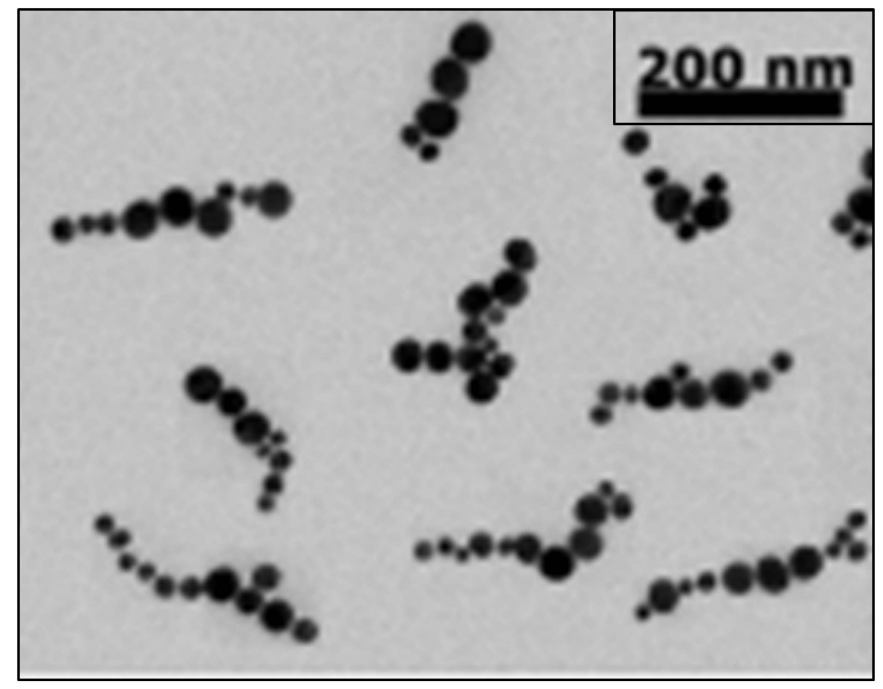


Figure 6

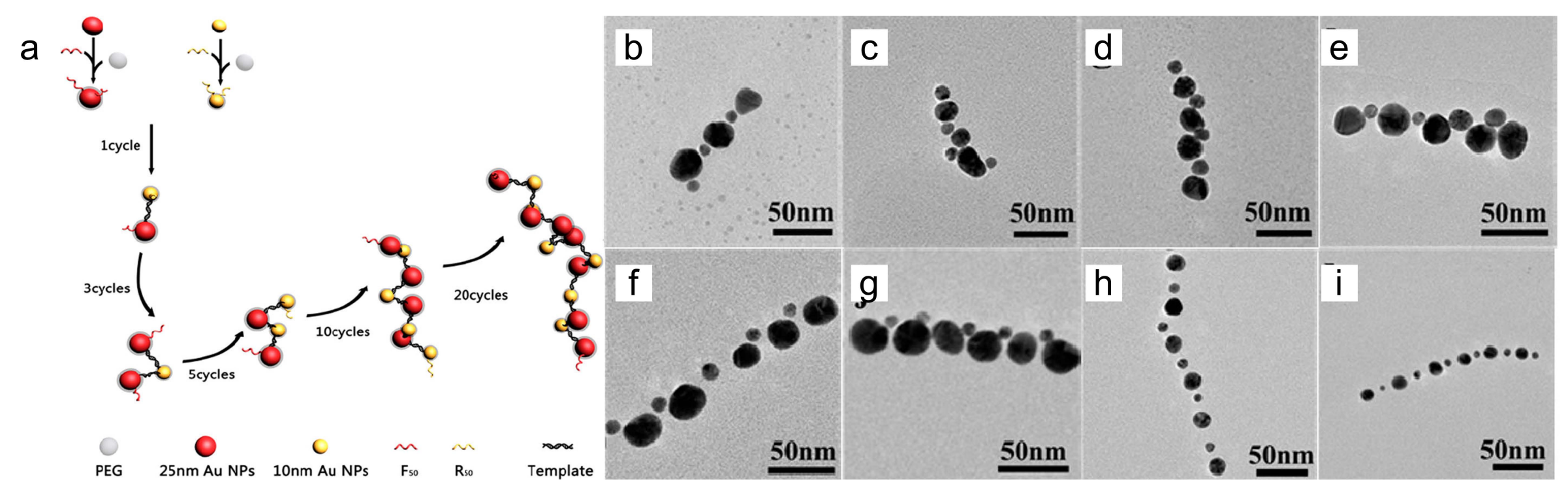


Figure 7

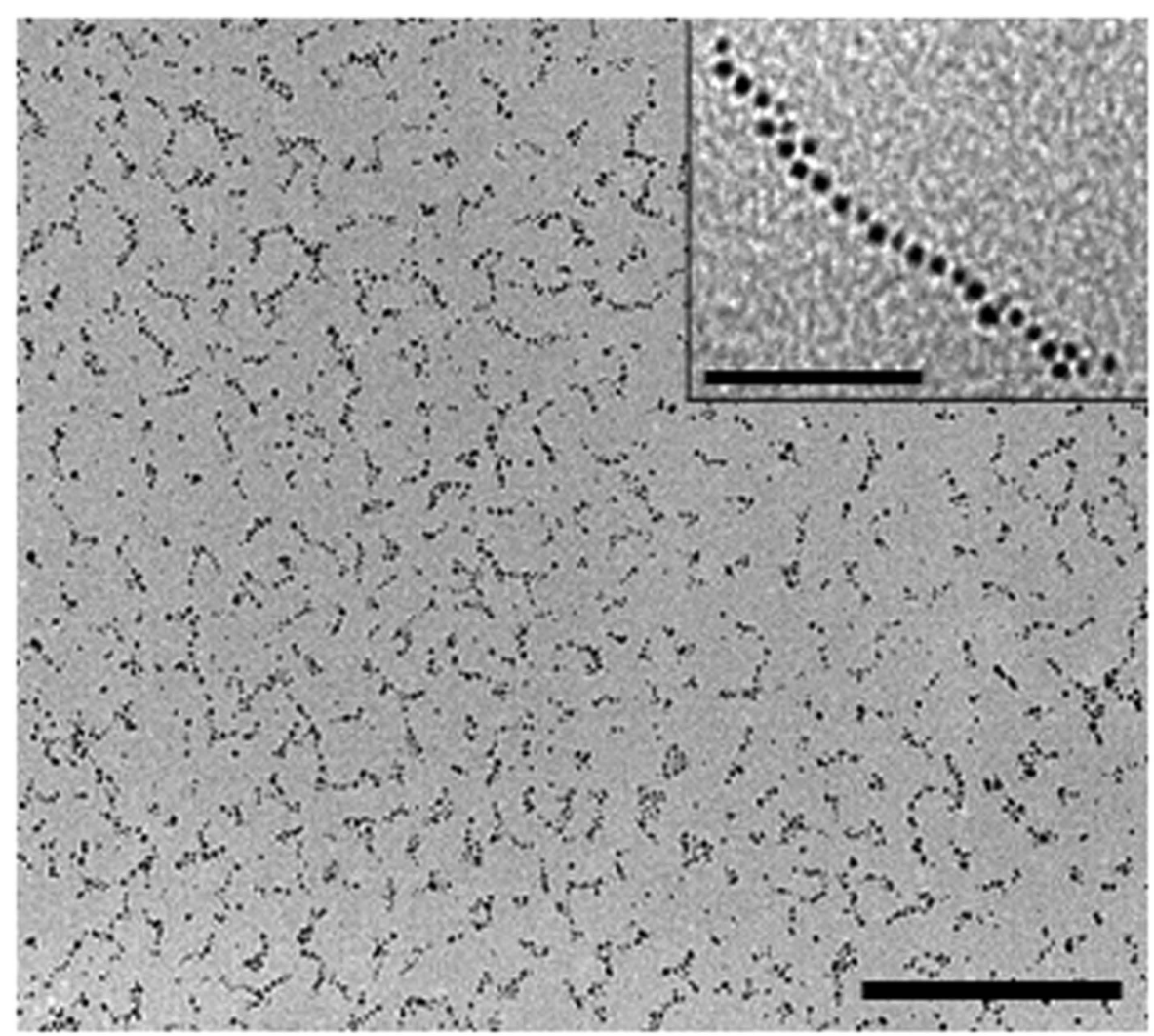


Figure 8

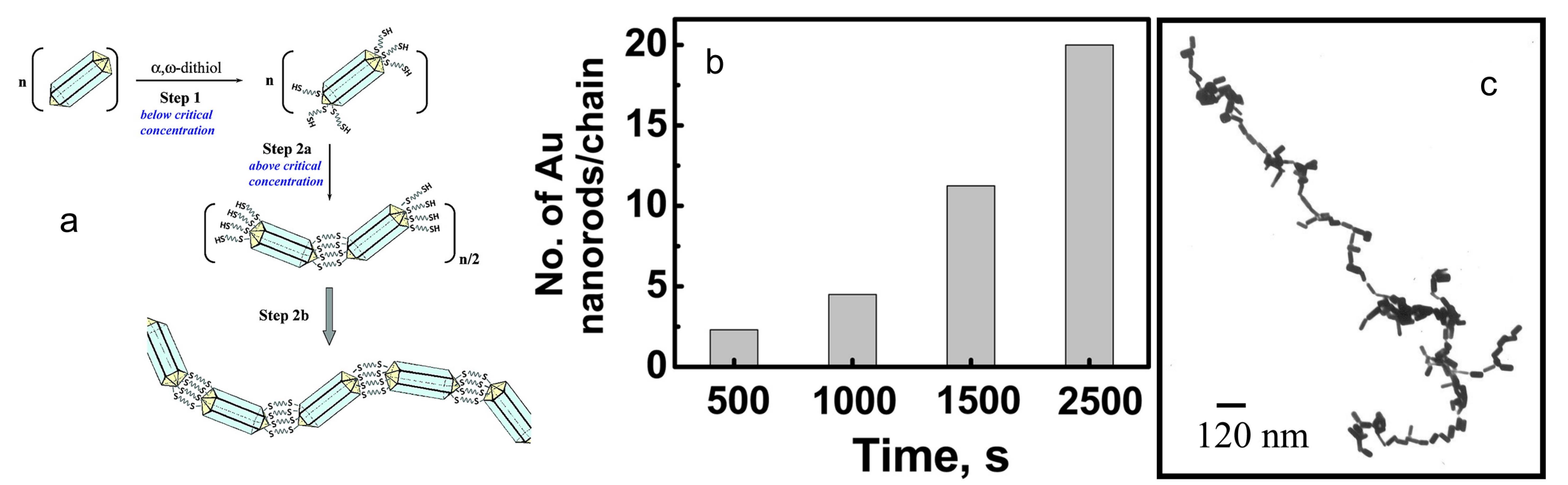


Figure 9

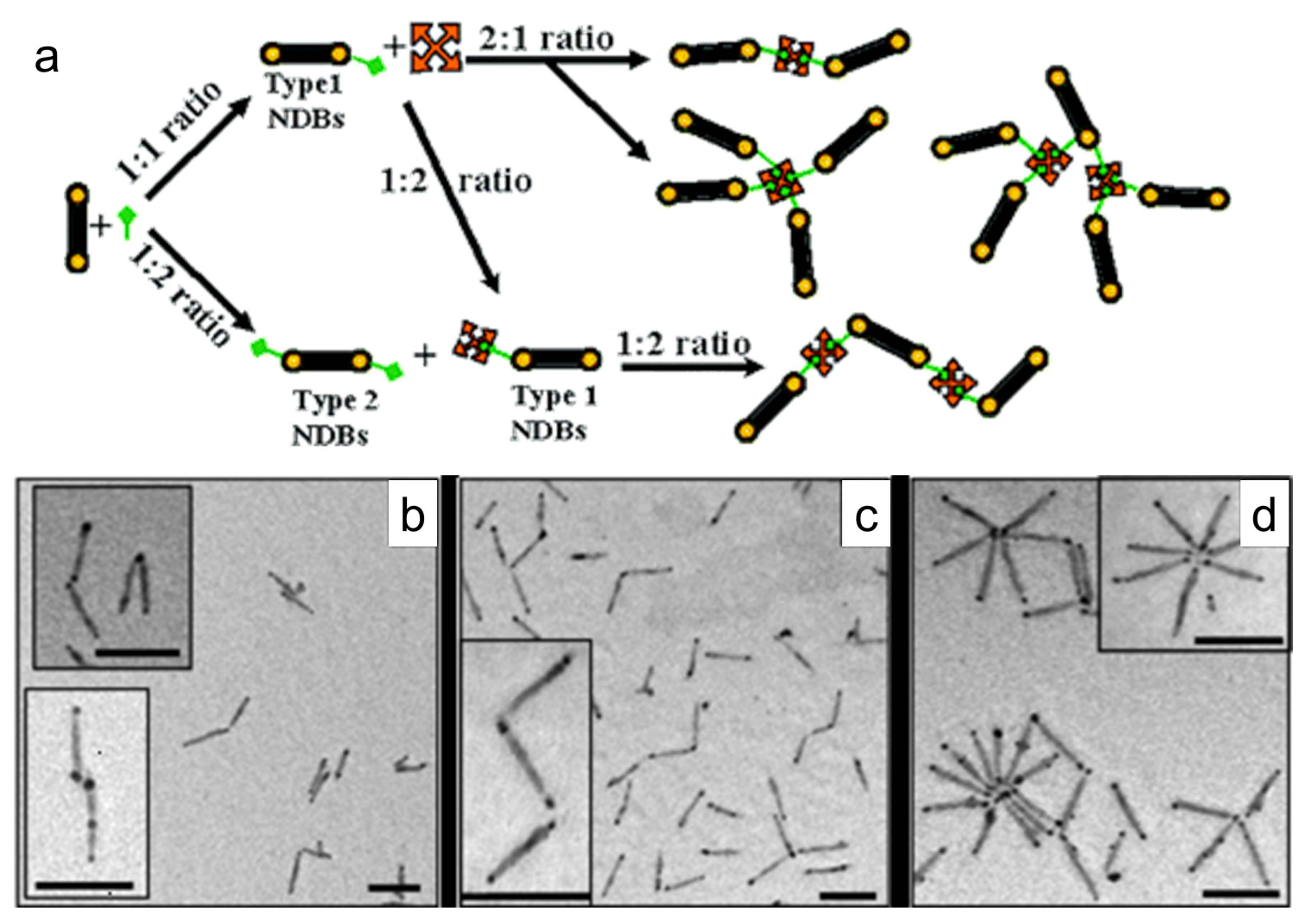




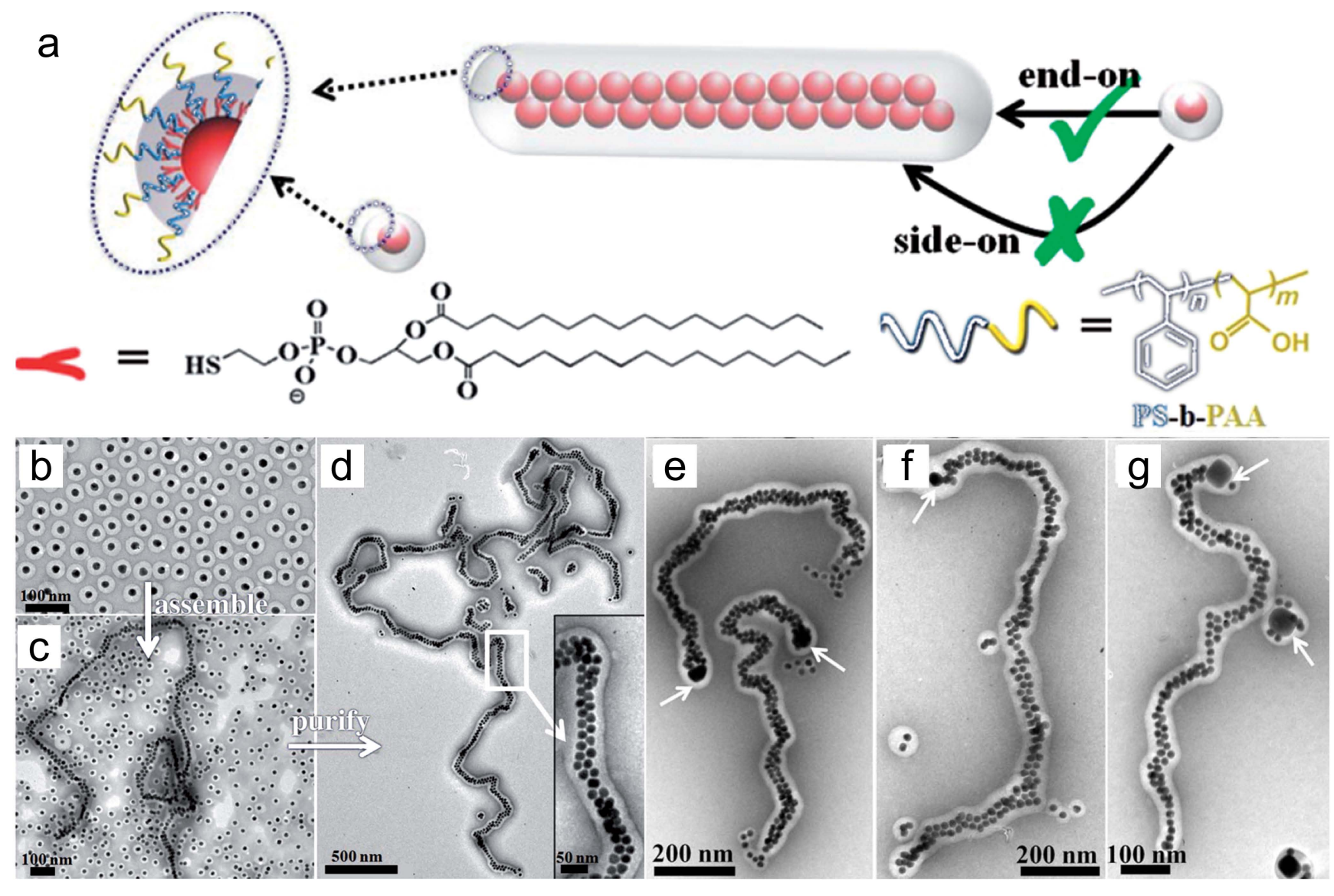


Figure 11

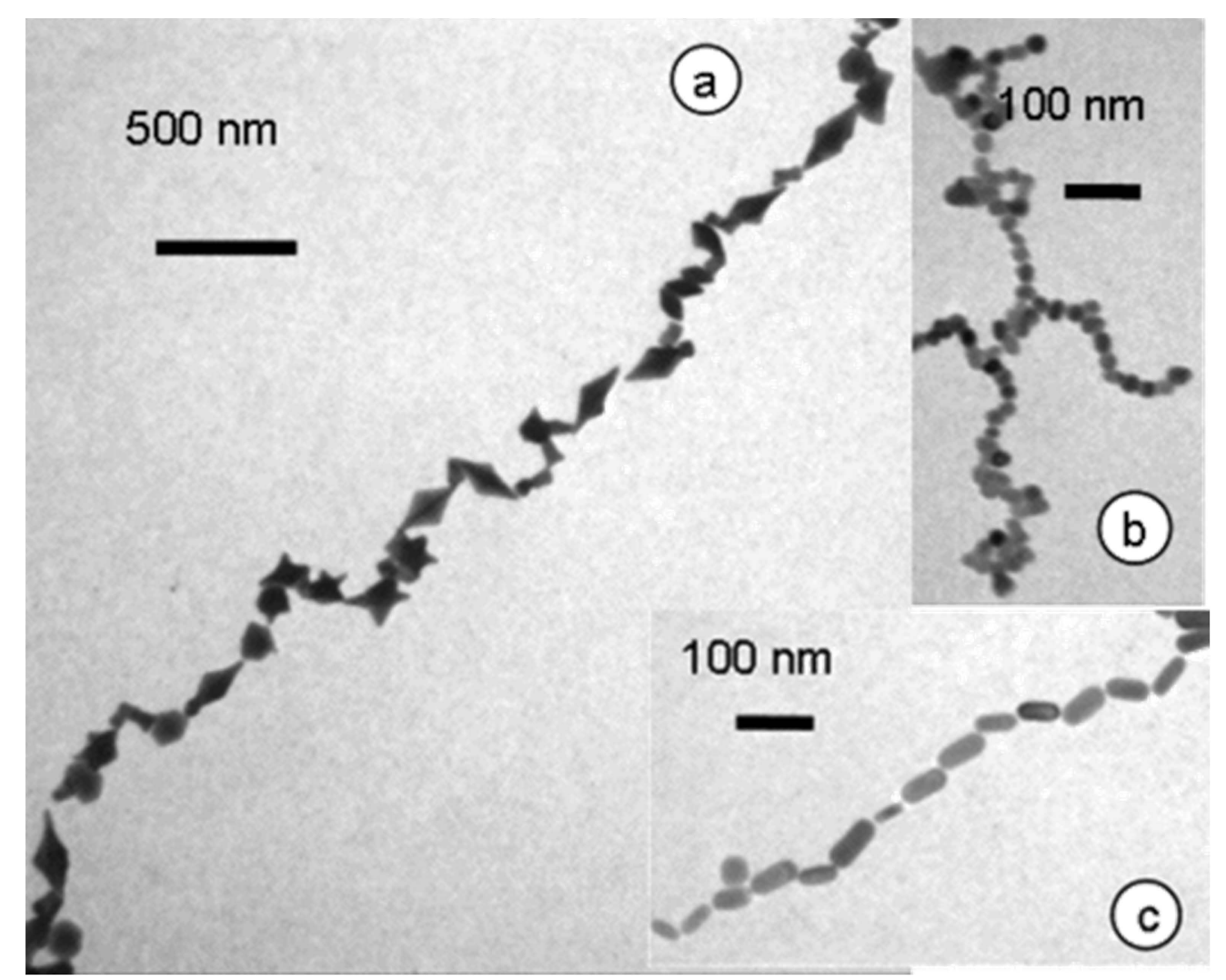




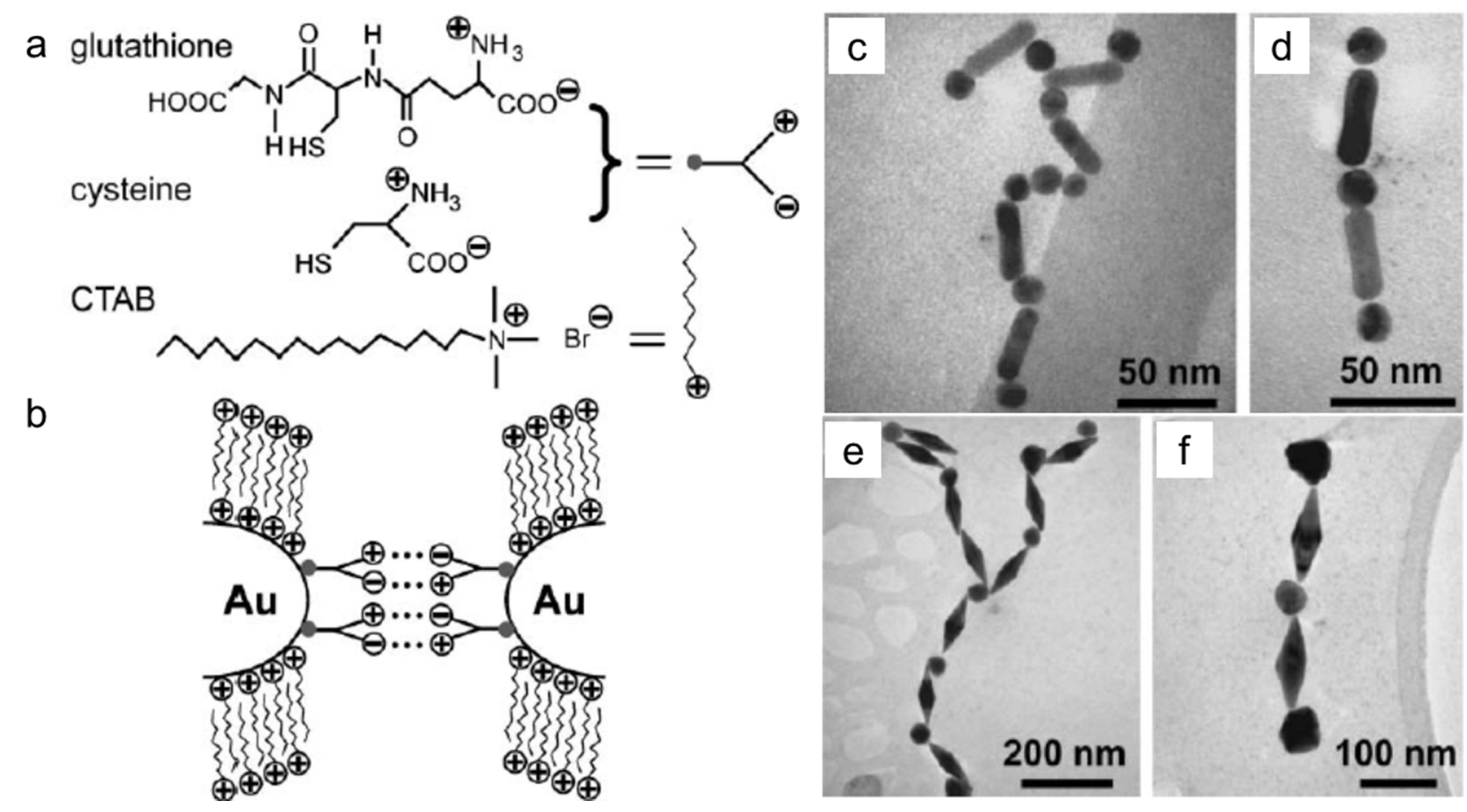


Figure 13

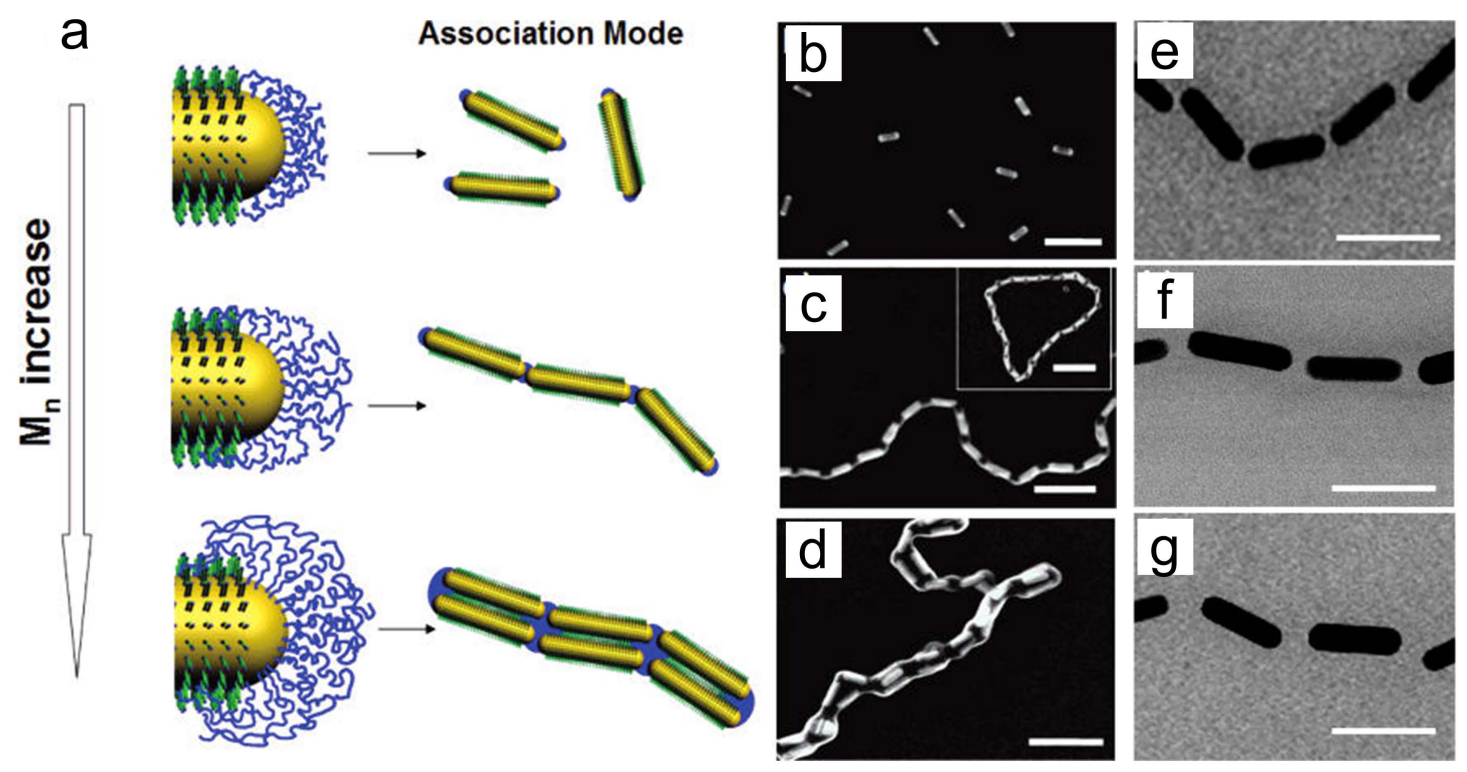


Figure 14

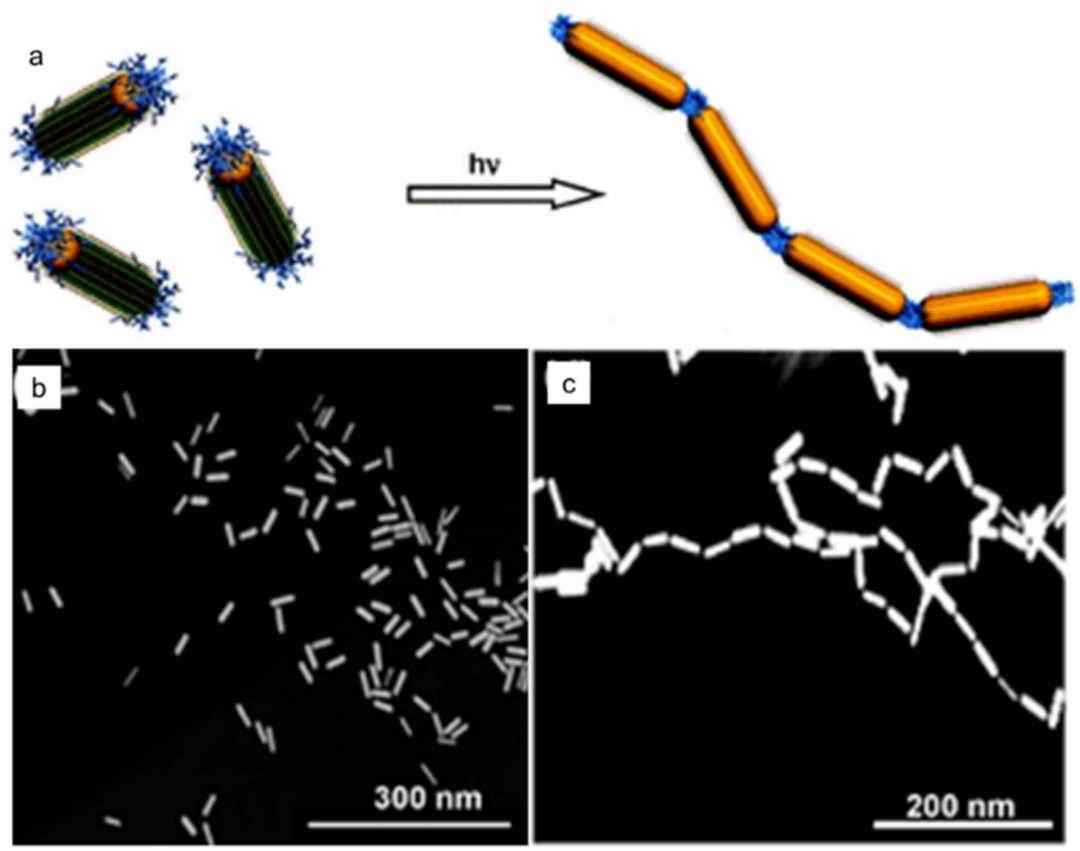


Figure 15

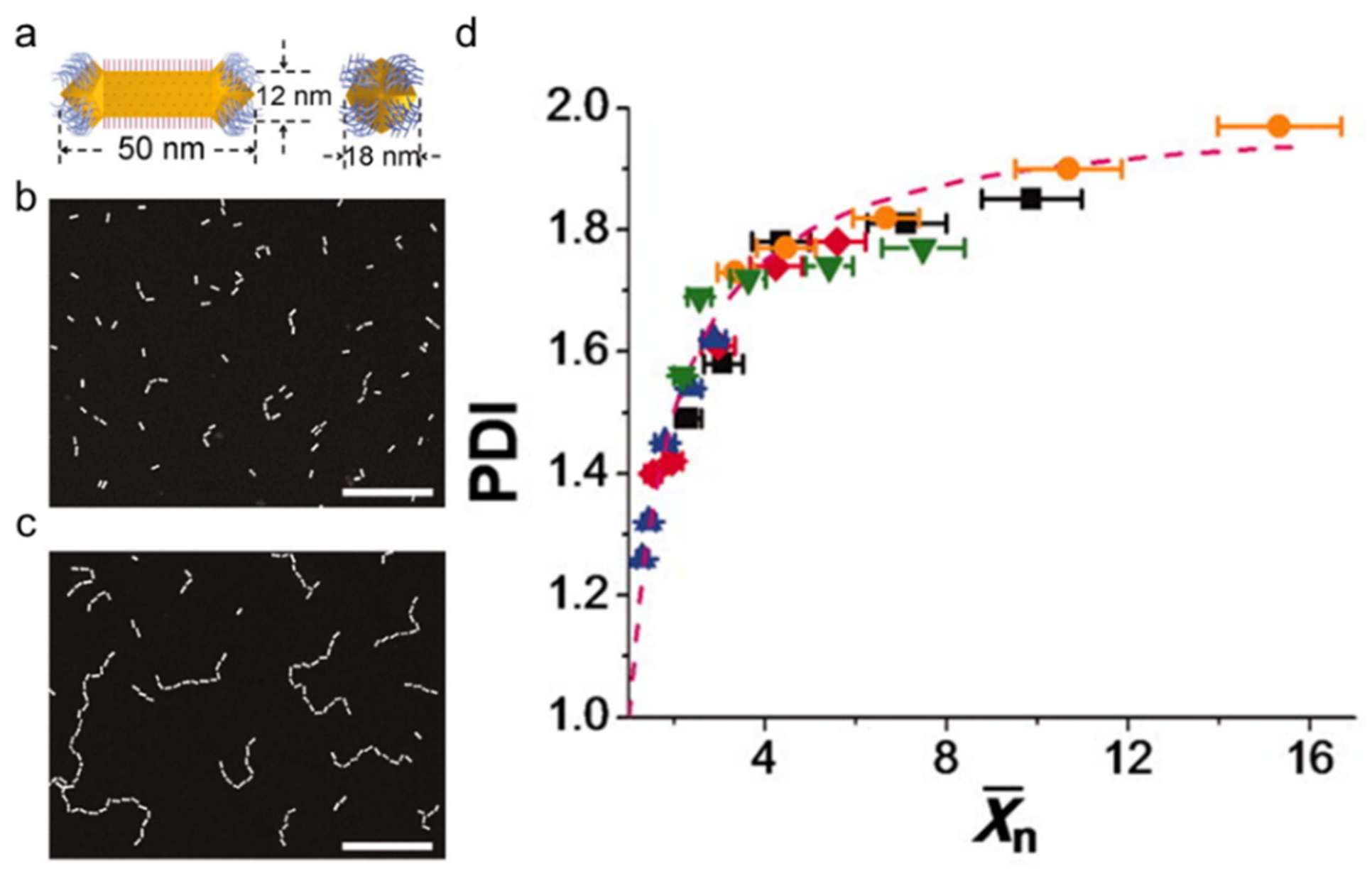


Figure 16

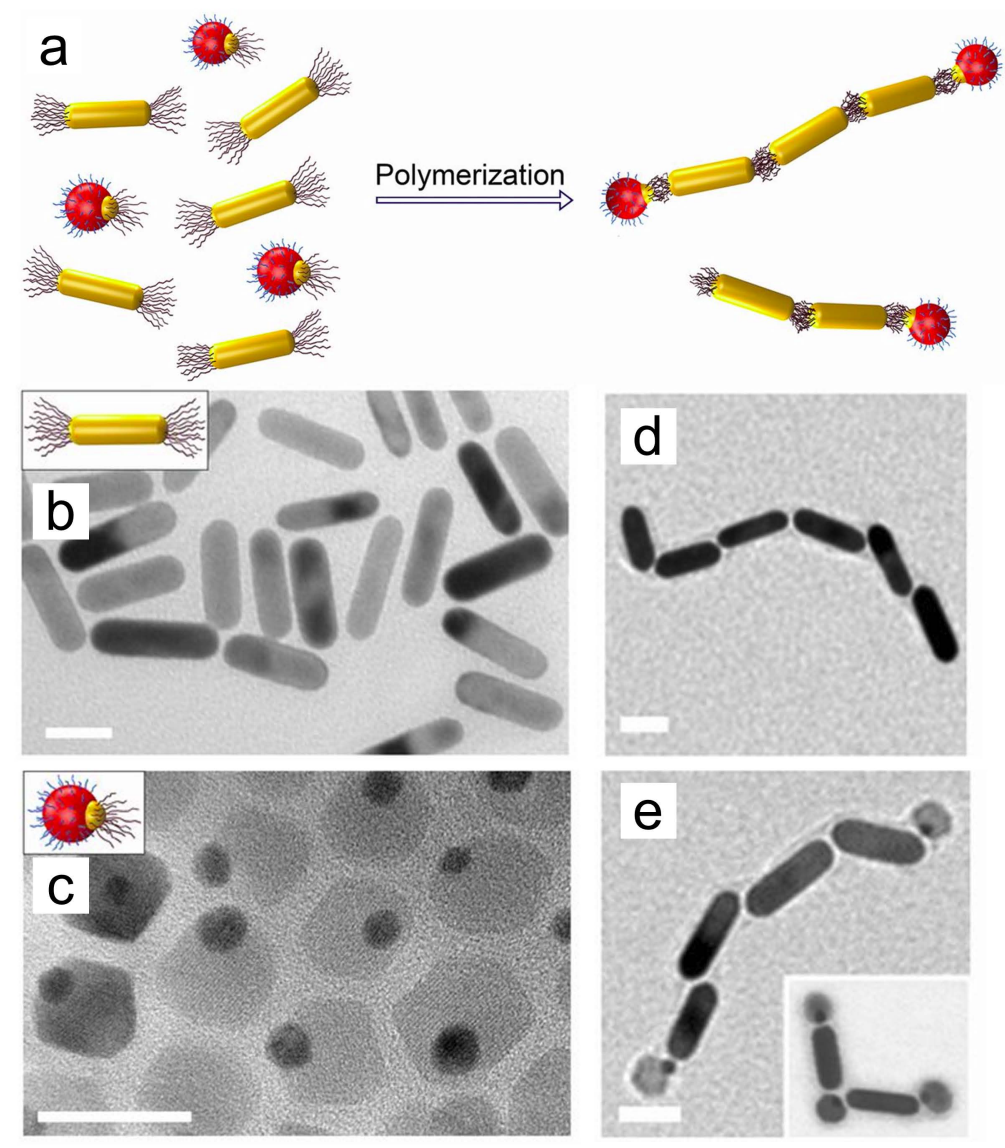


Figure 17

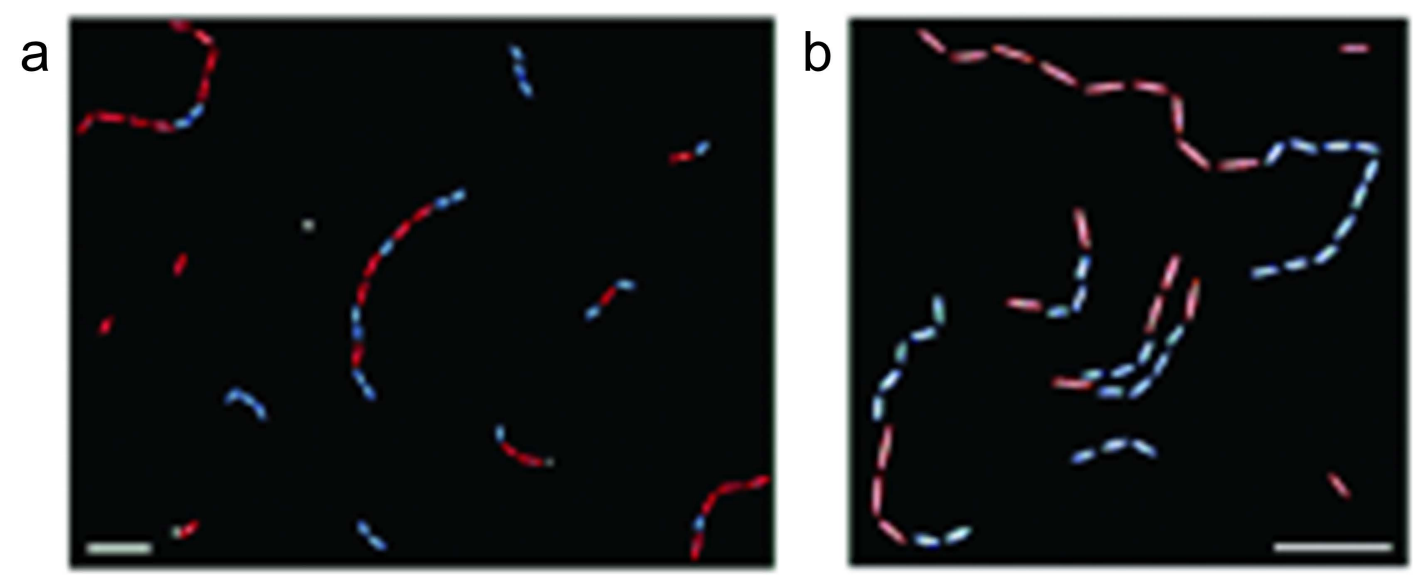


Figure 18

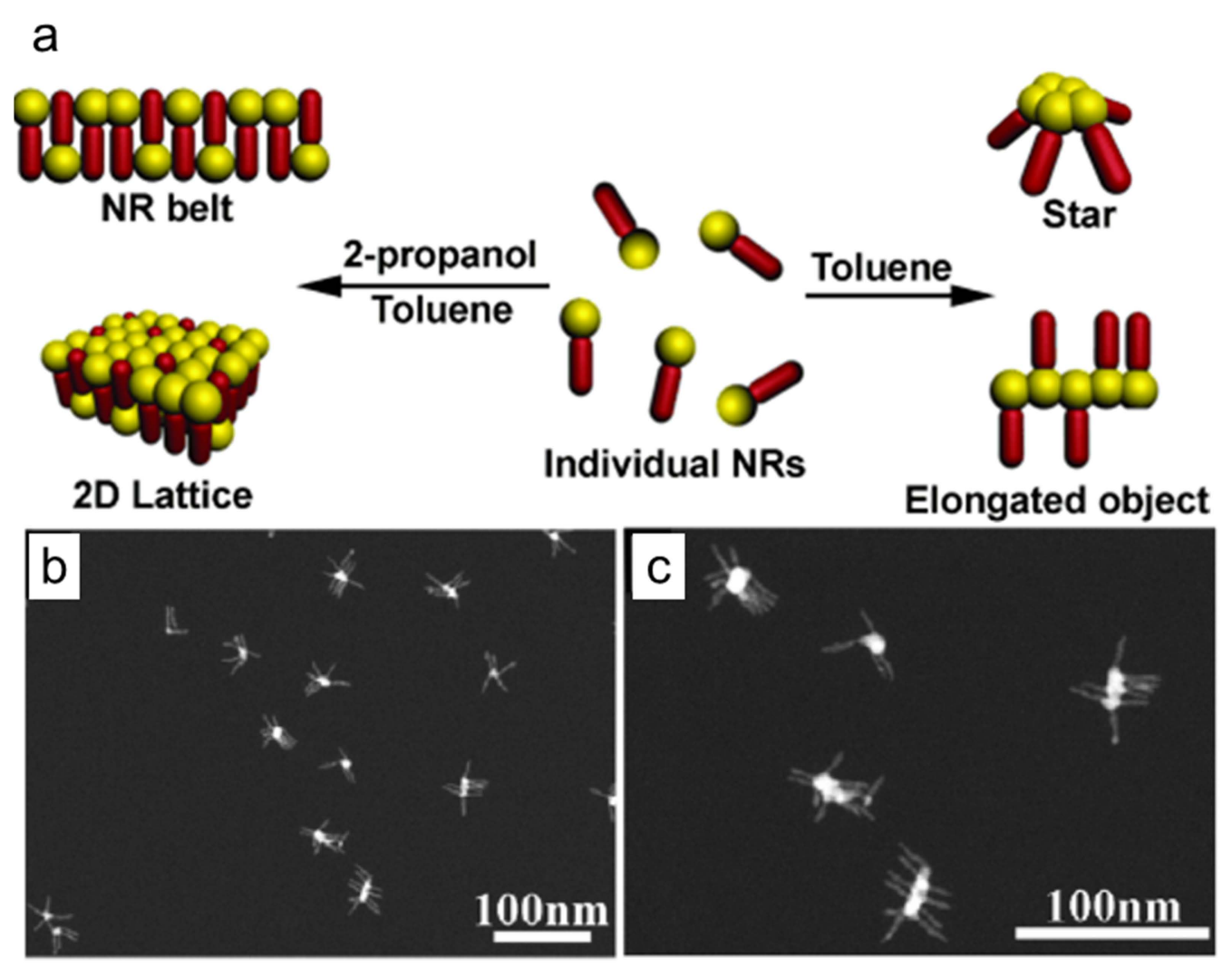


a
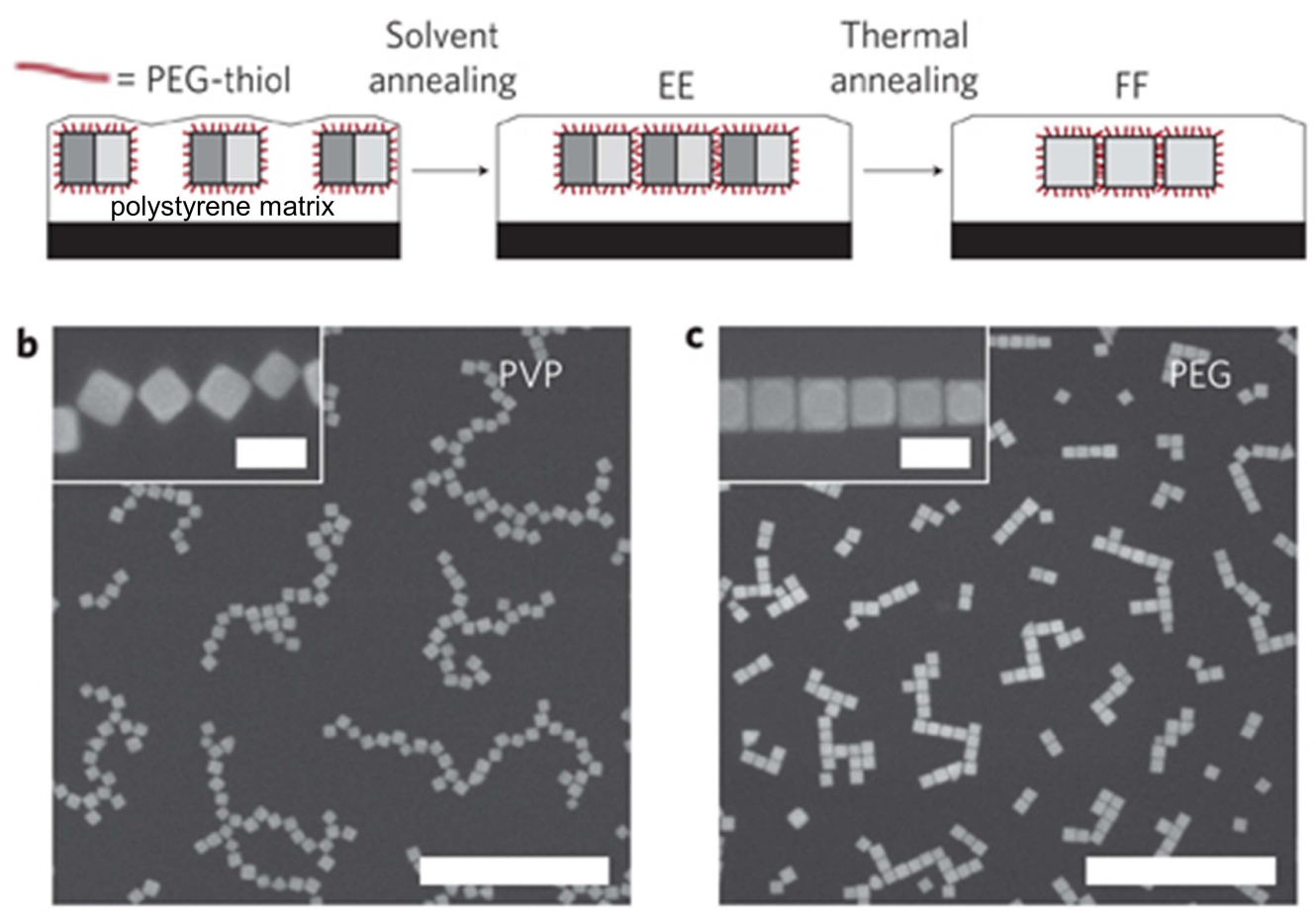
Figure 20

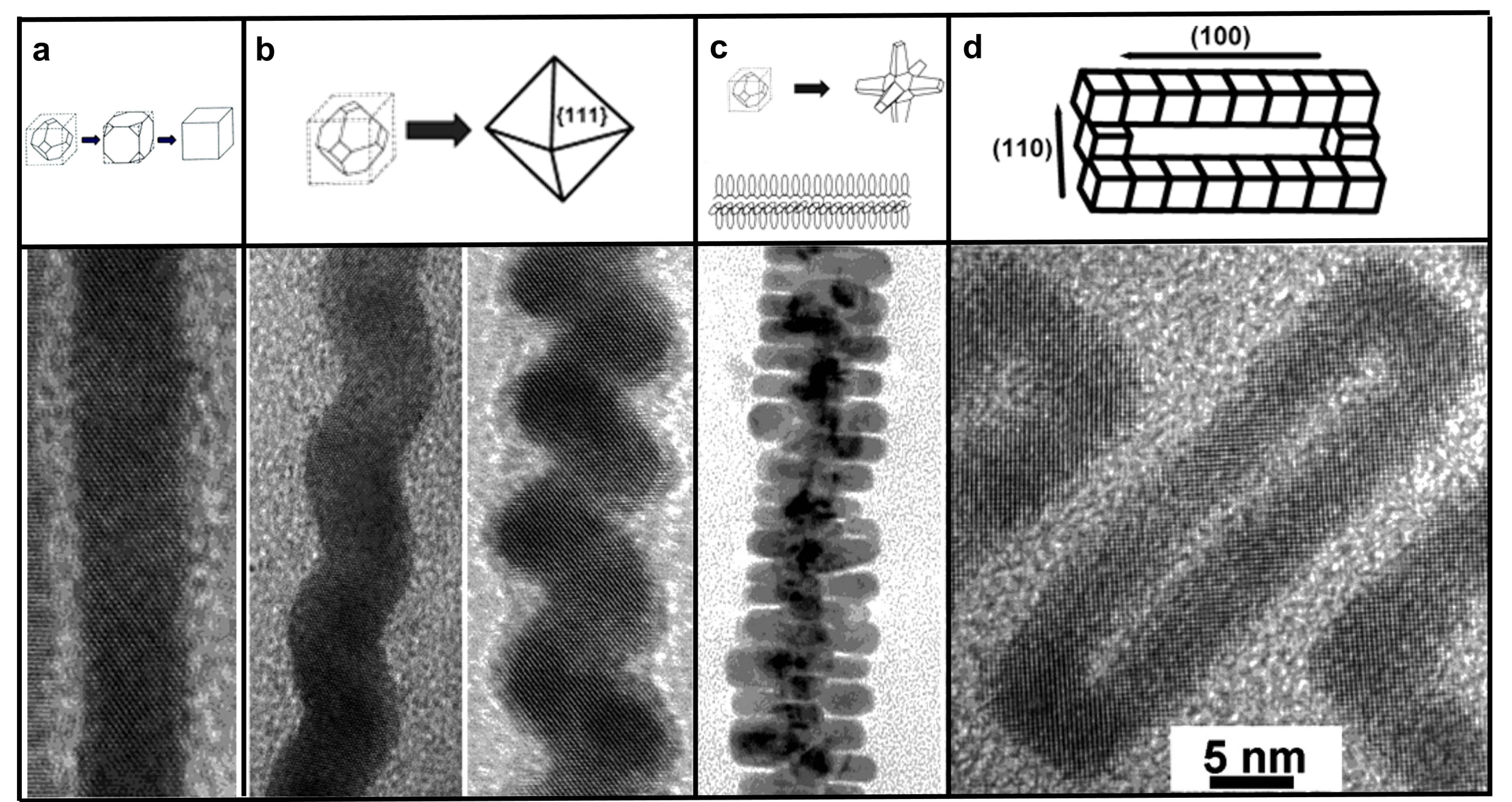




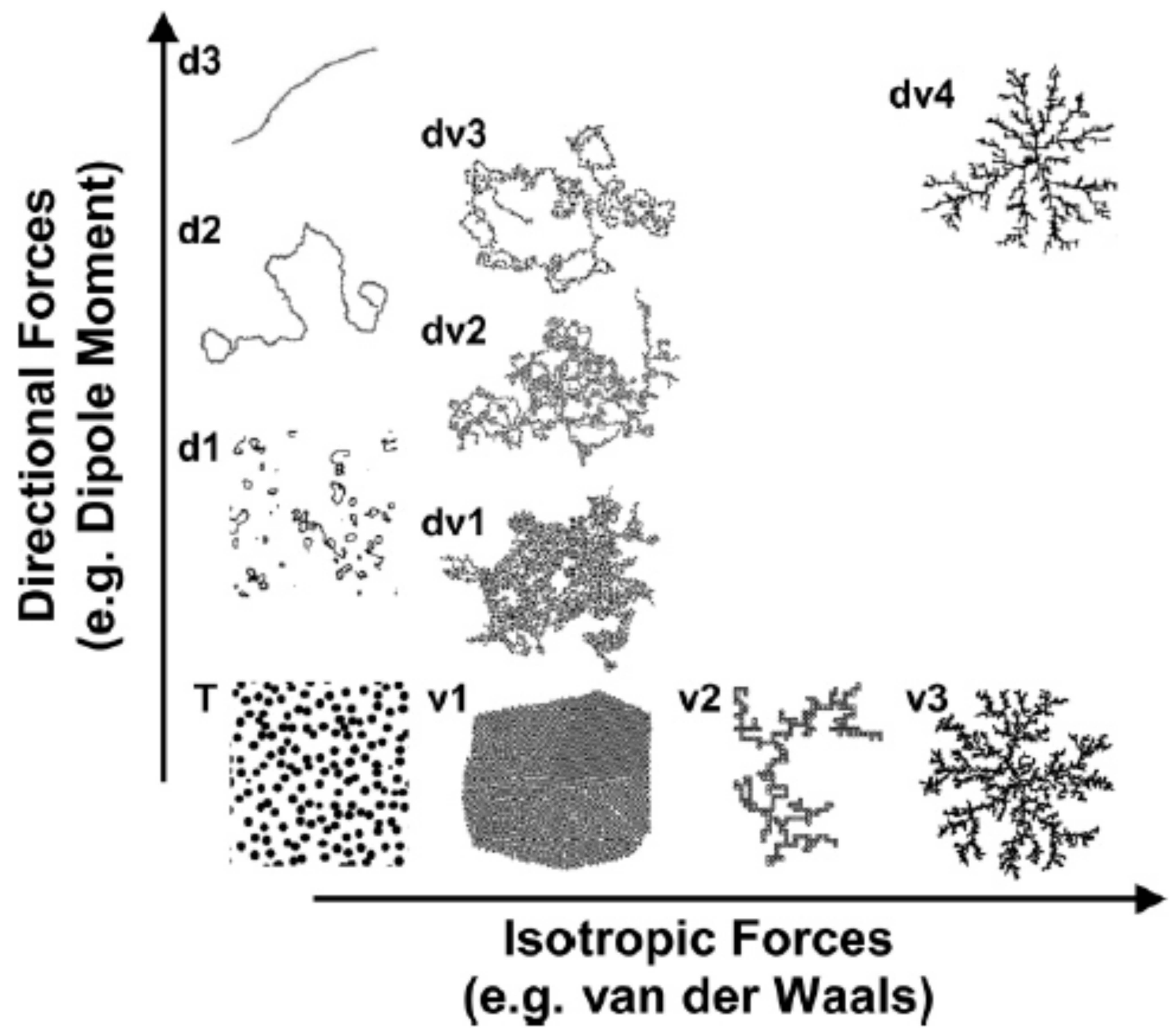


Figure 23

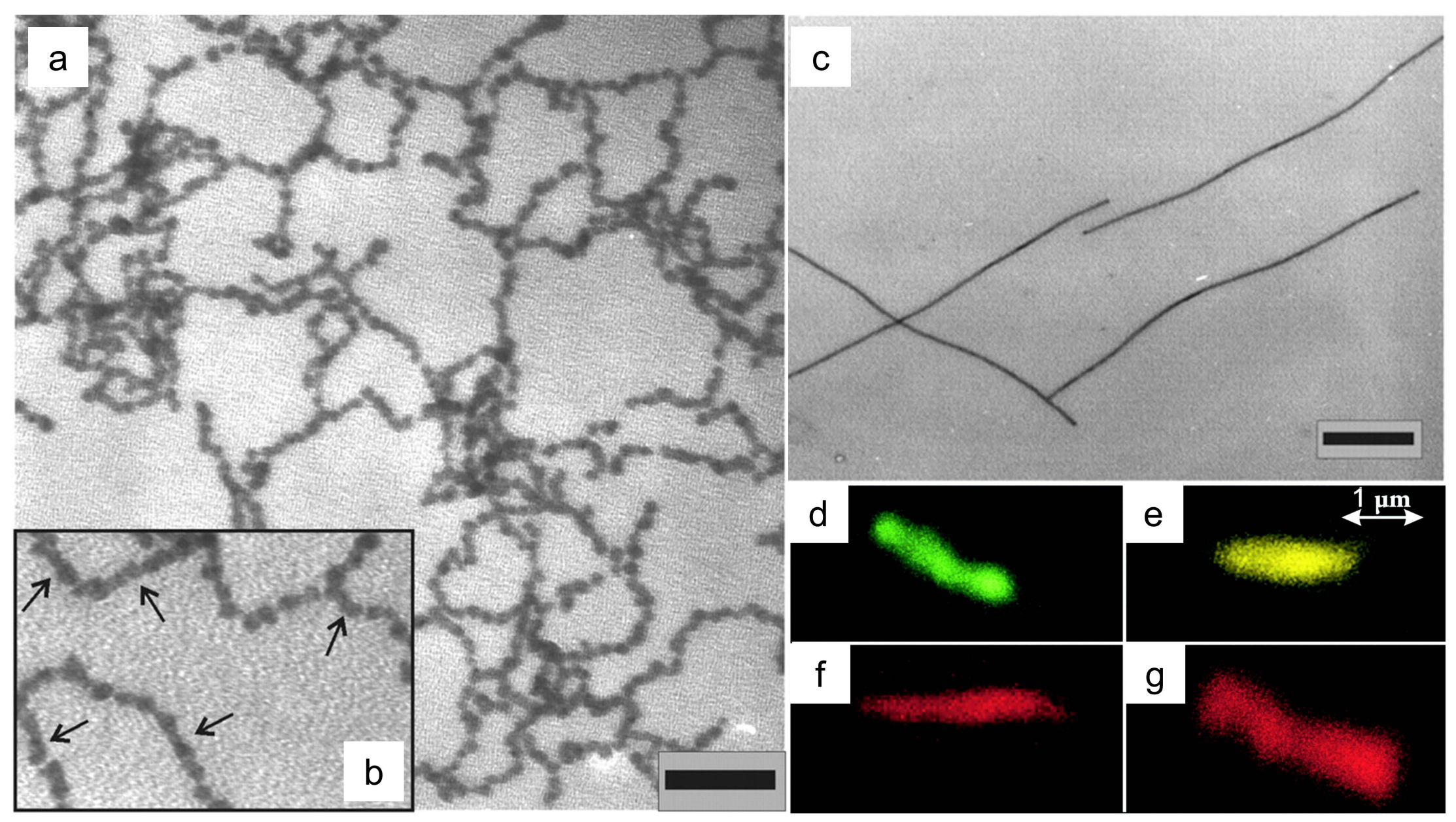


Figure 24
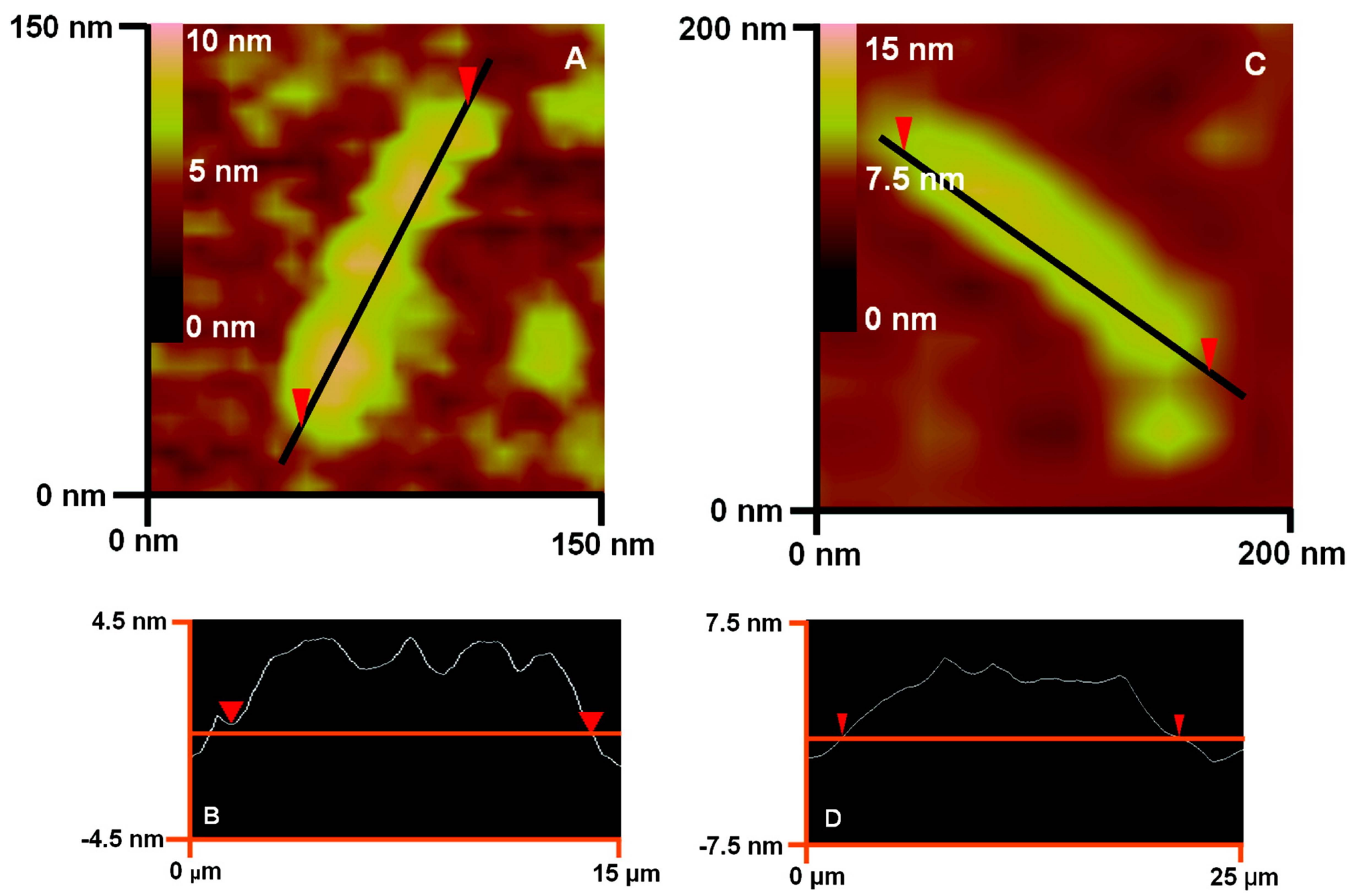
Figure 25
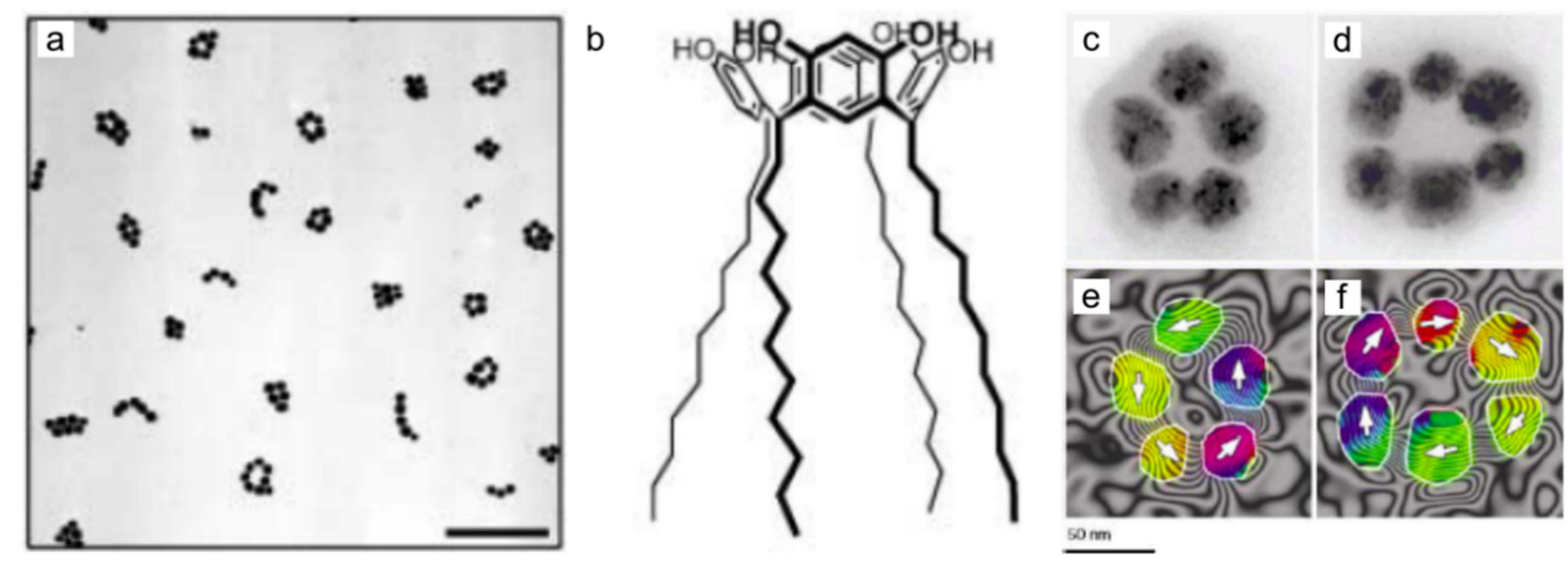
Figure 26

a

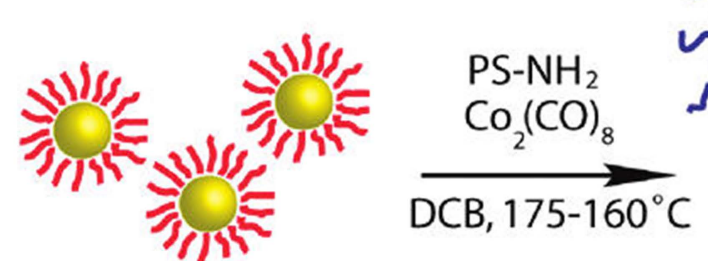

Oleylamine Capped AuNPs

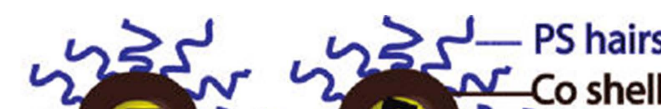

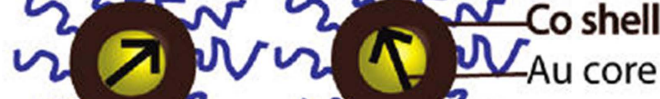

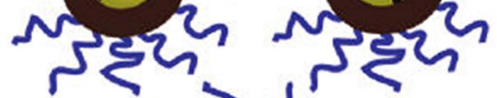
पूर्यु पूर उदू?

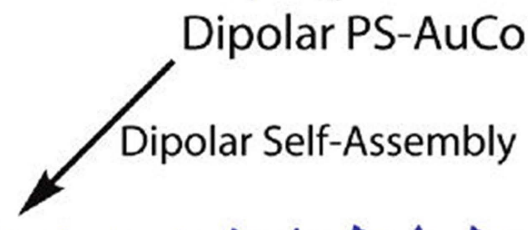

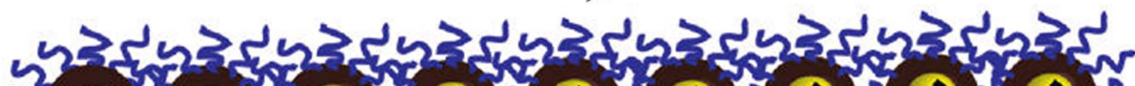

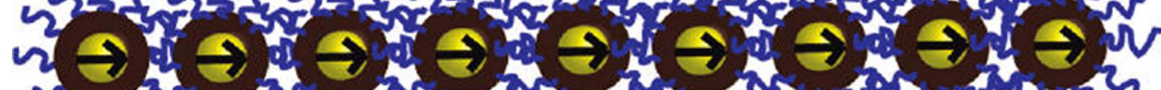

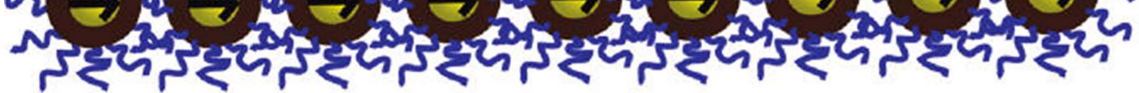

Pre-Organized NP Chains
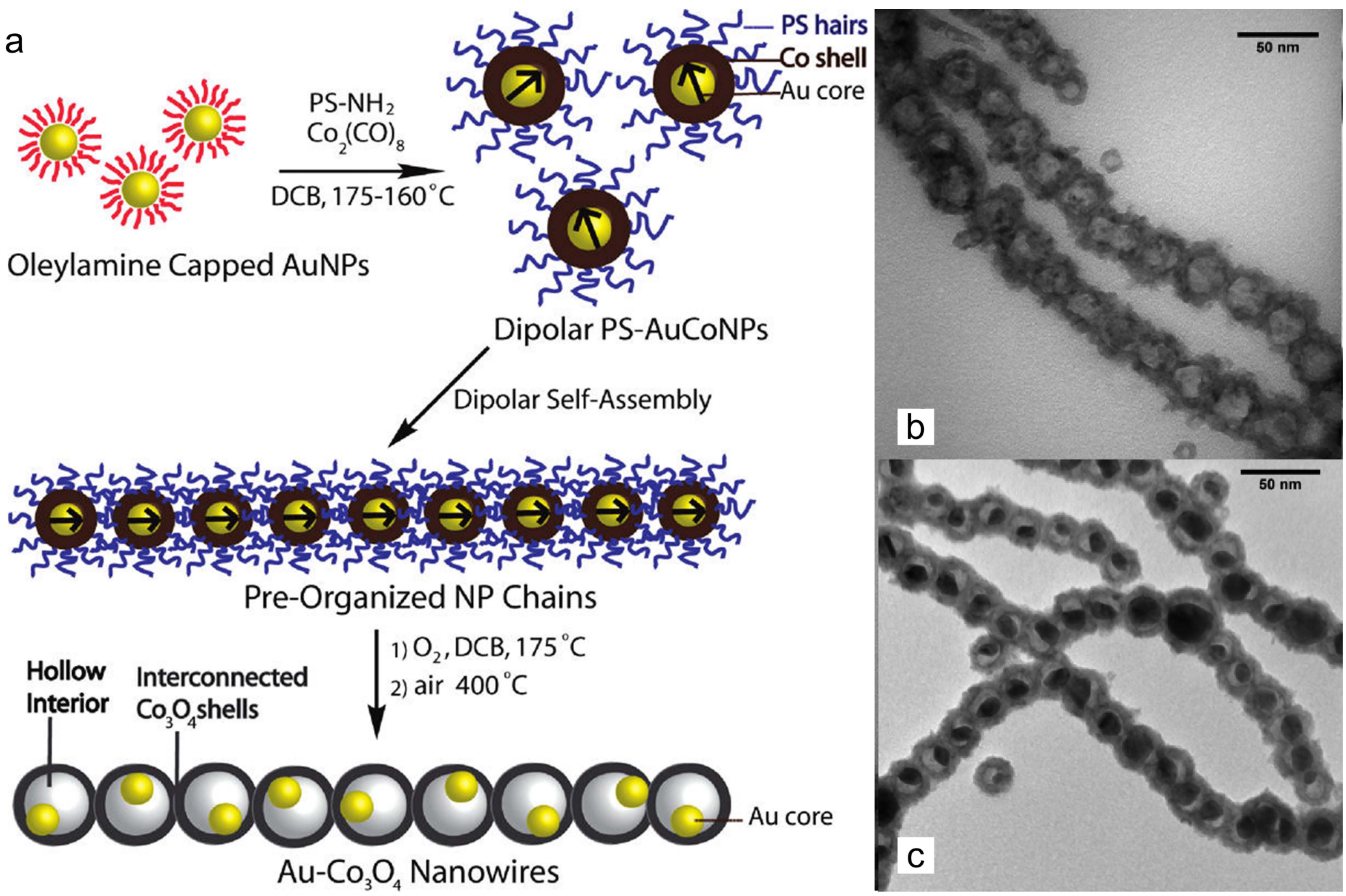
Figure 27

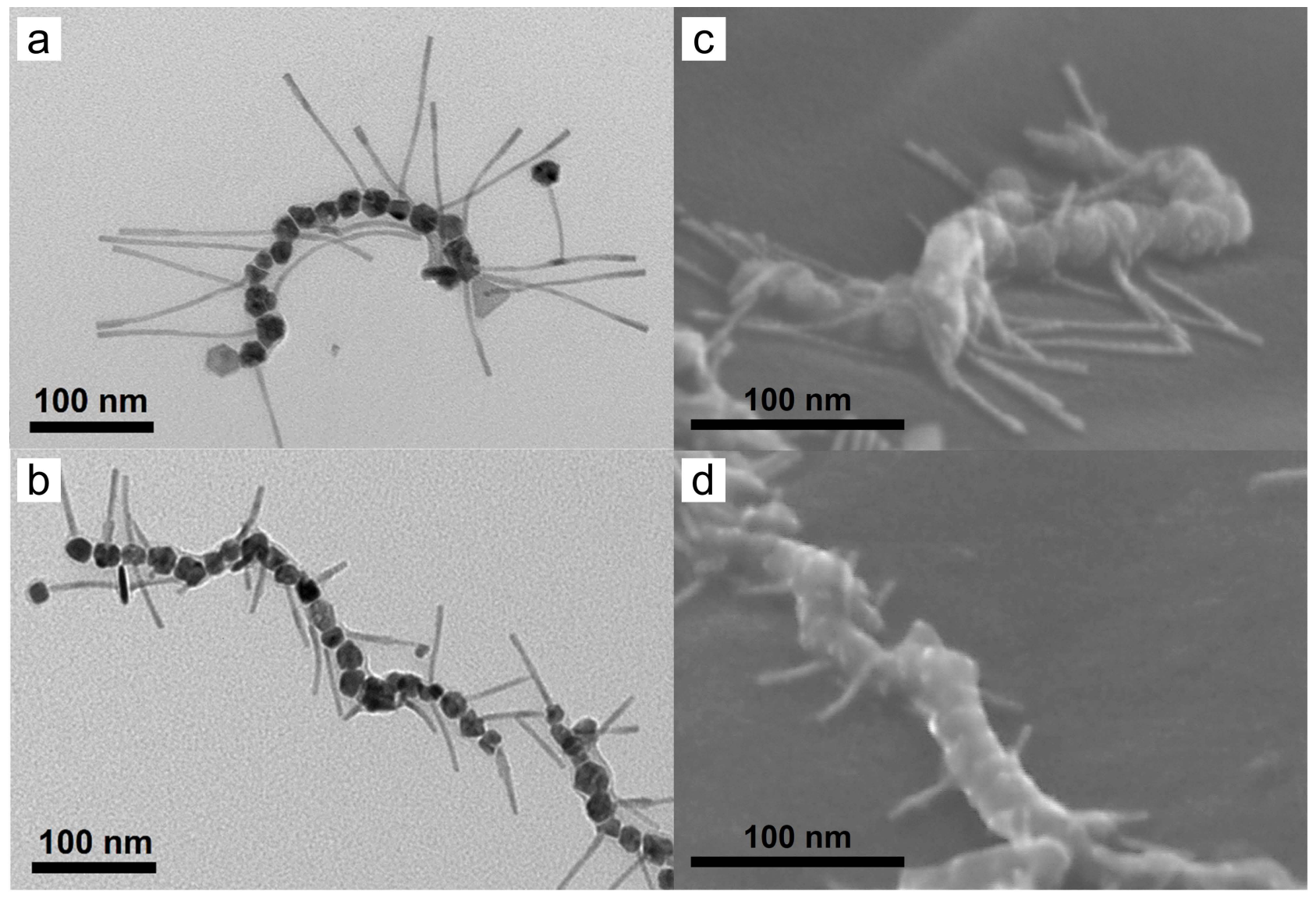


a

Dipolar CoNPs Dipolar CoNPwithout nanorods tipped nanorods
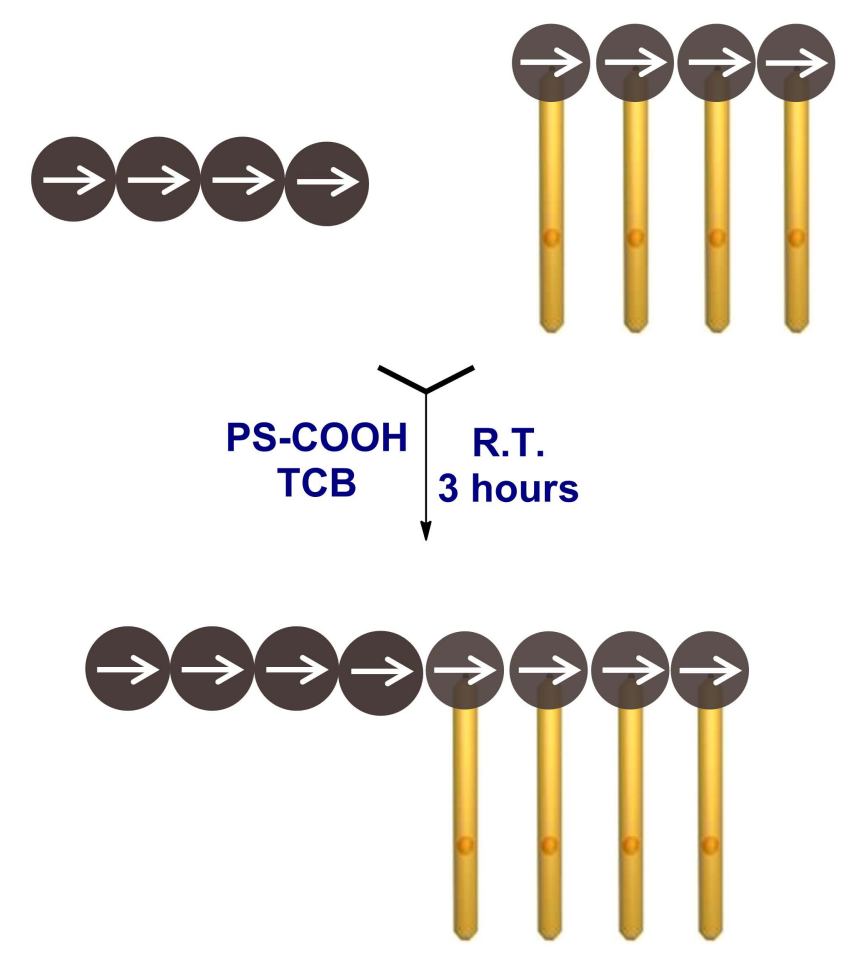

Segmented copolymer

* plus remaining homopolymers

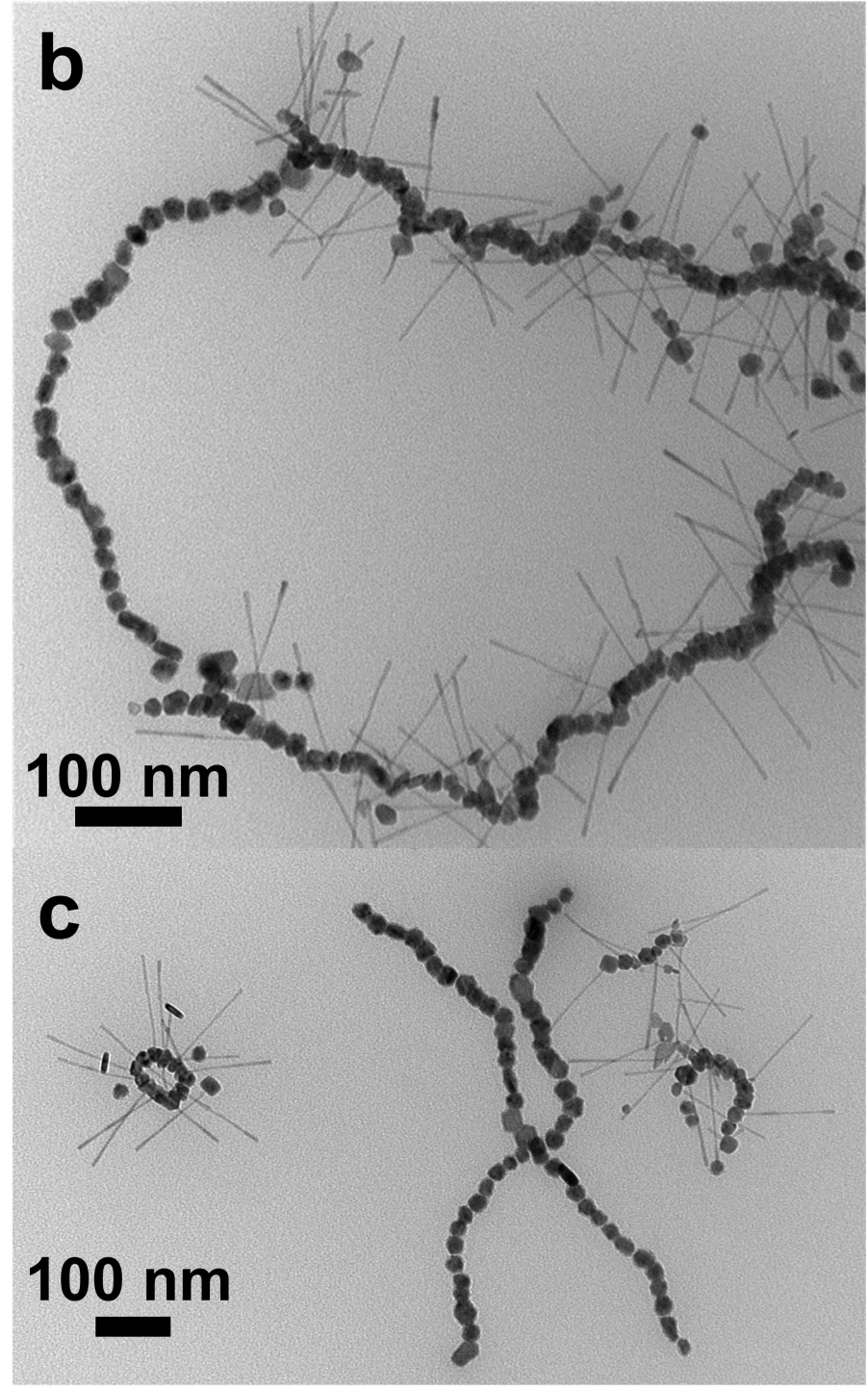


Figure 29

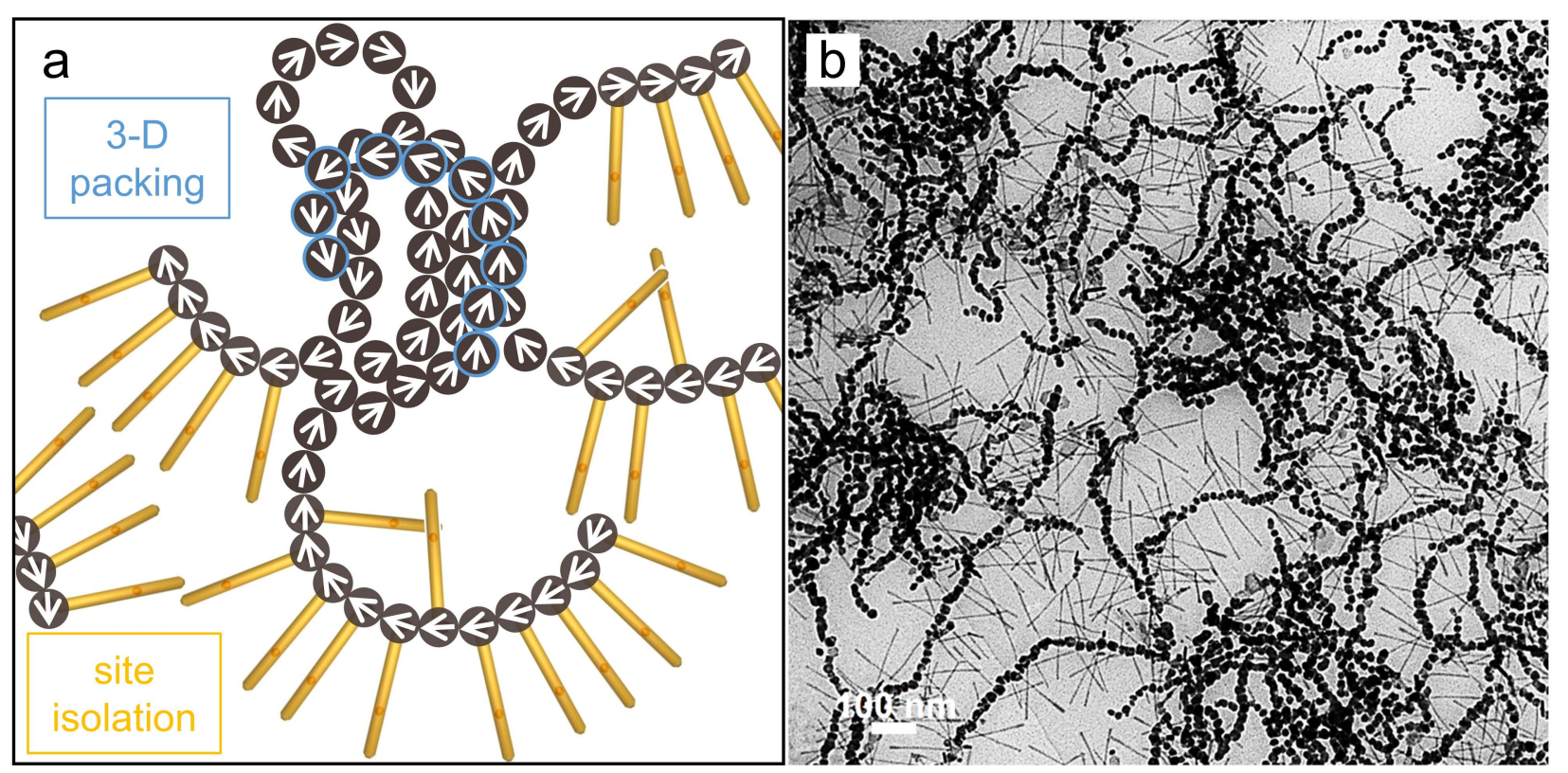


Figure 30

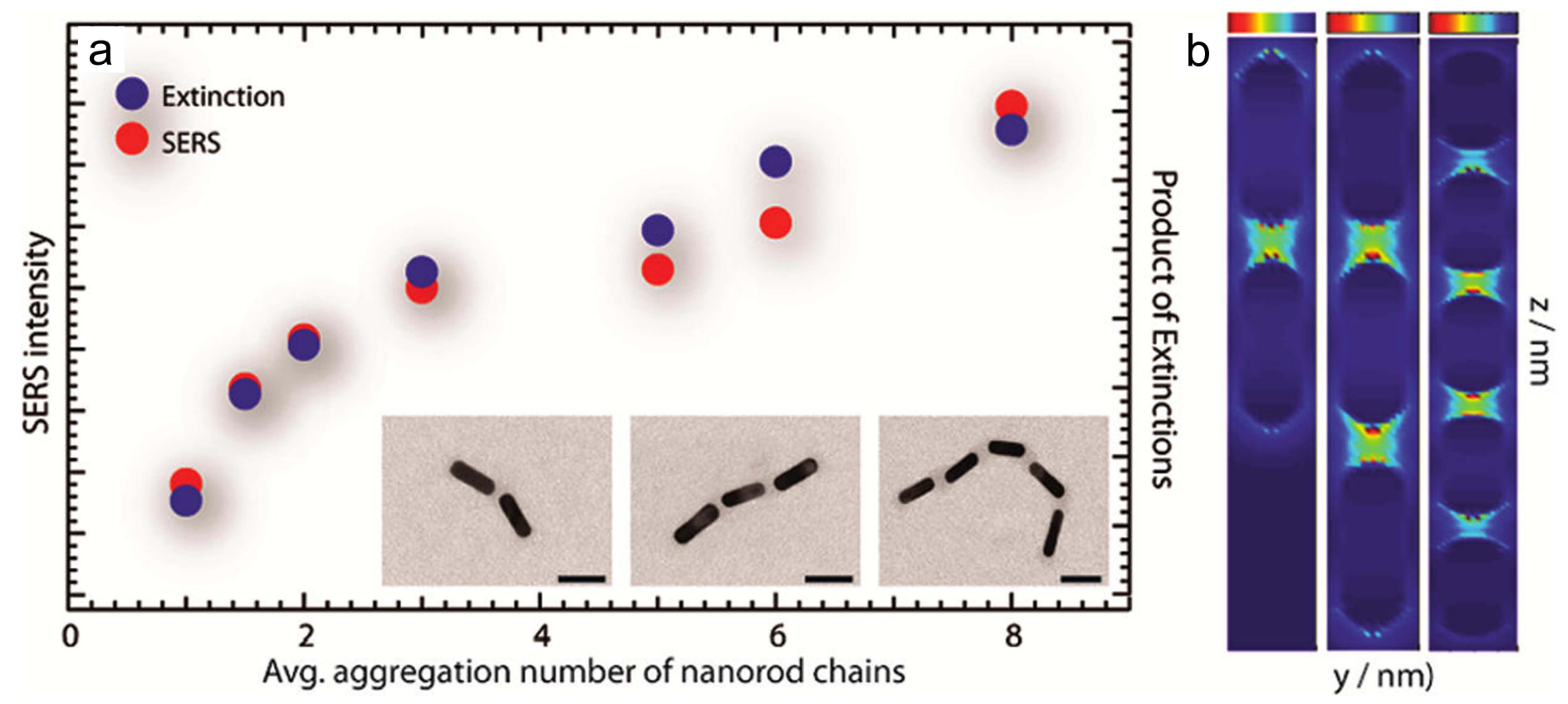


Figure 31
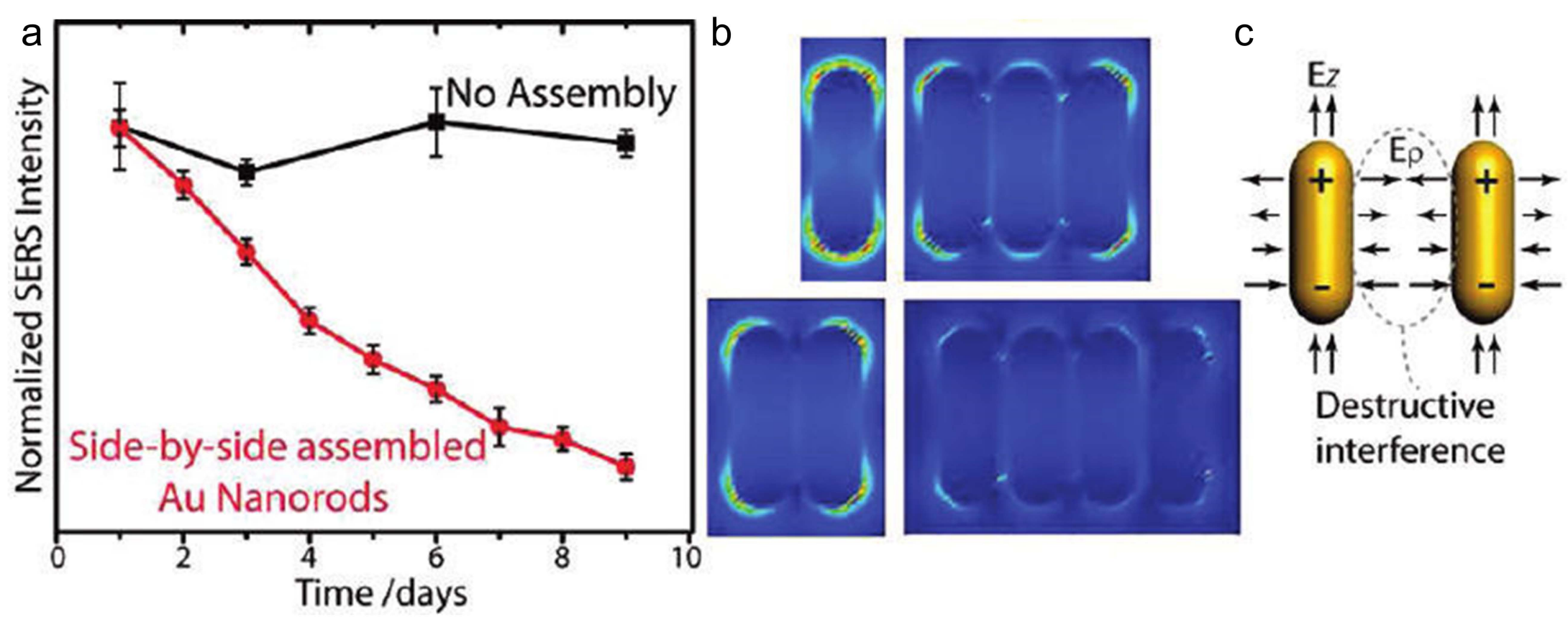


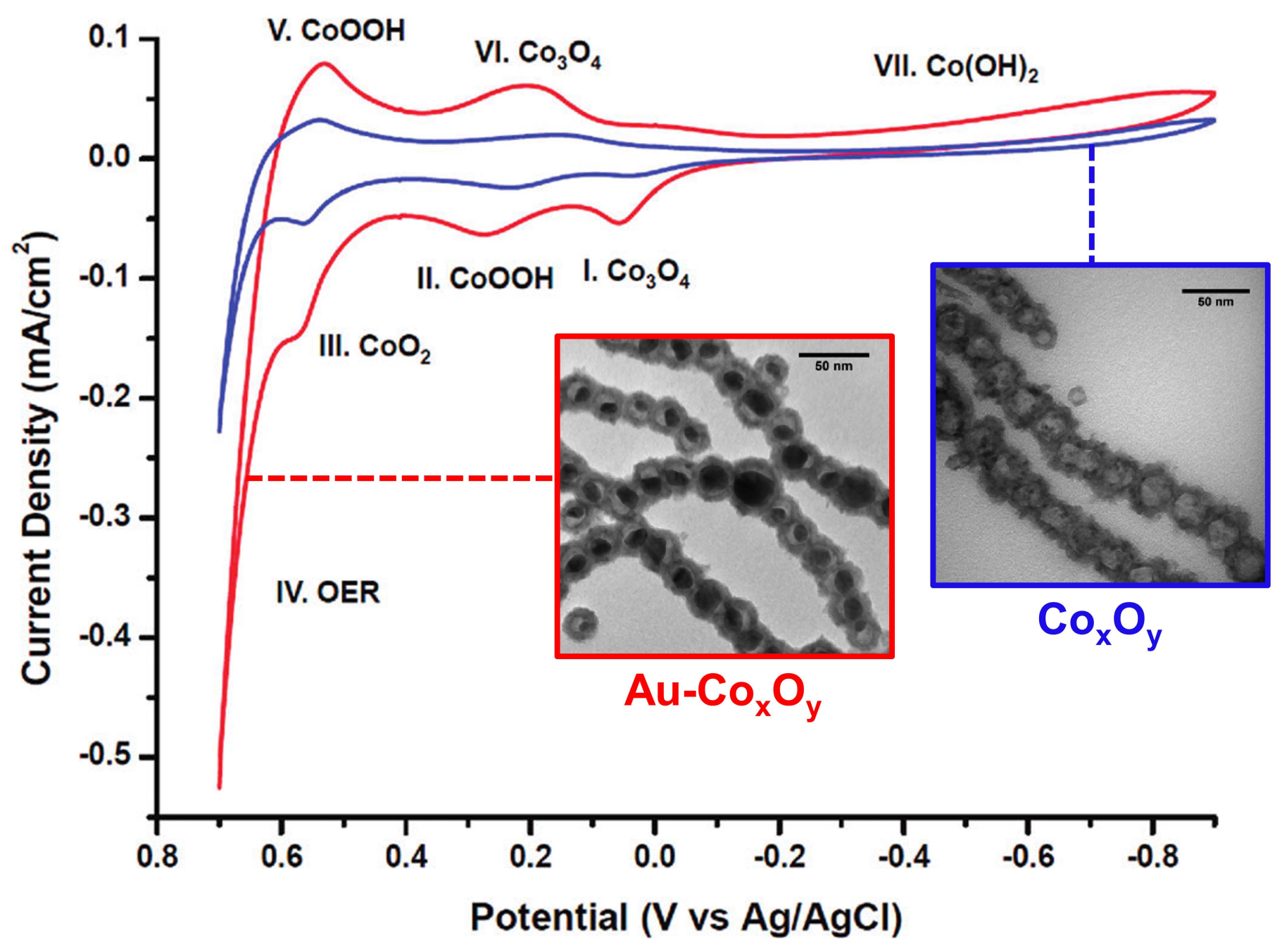




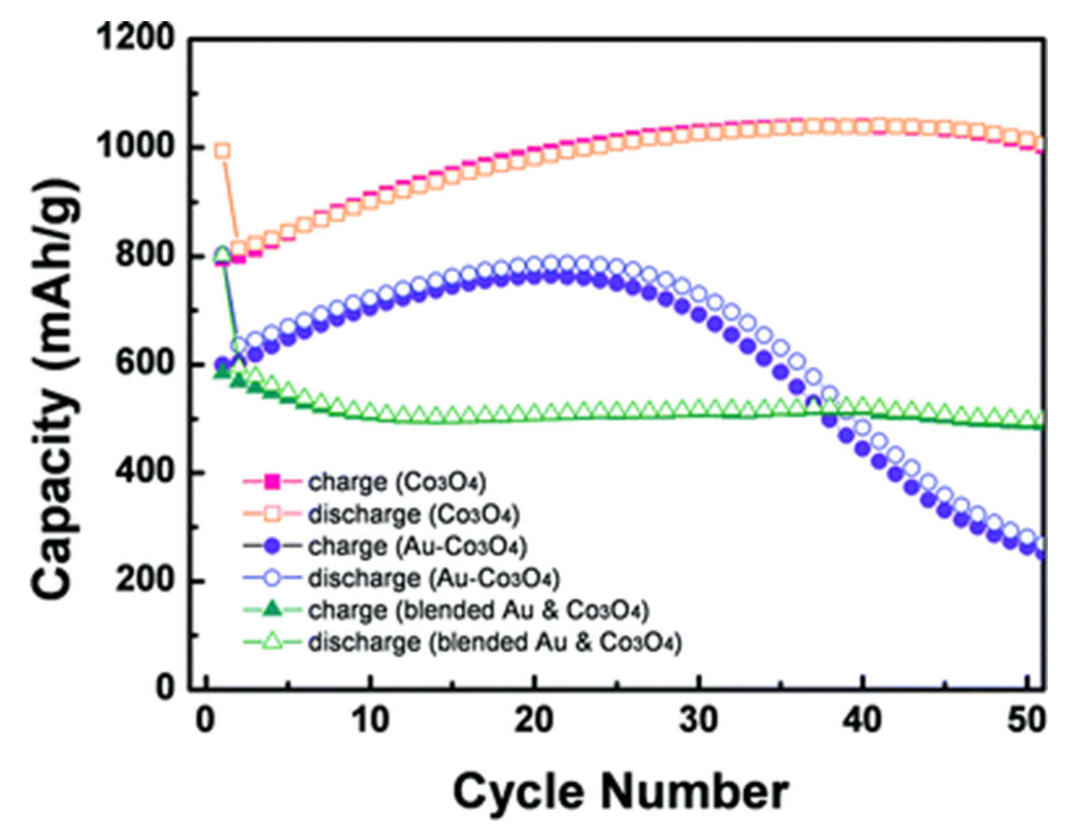


a) Ligand-directed

\section{organic ligands}$$
\text { in }
$$

inorganic core

b) Surface-directed

3) non-covalent

2) multifunctional ligands

1) oriented lattice fringes attachment

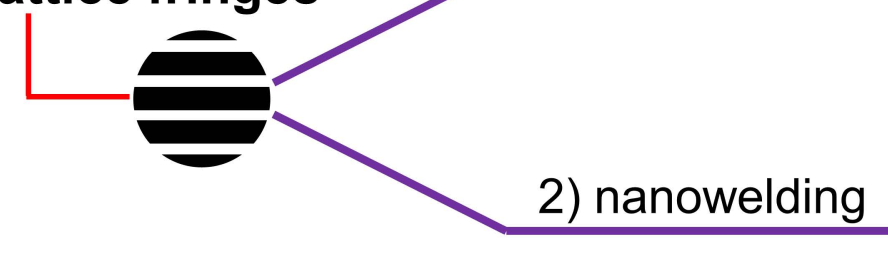

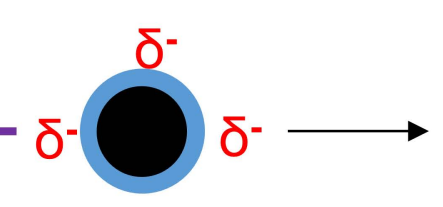<smiles></smiles><smiles>[X]CCNC(=[V])c1ccccc1</smiles>

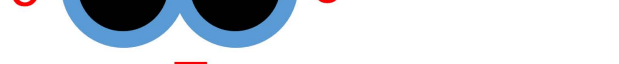

$\delta^{-}$<smiles></smiles>

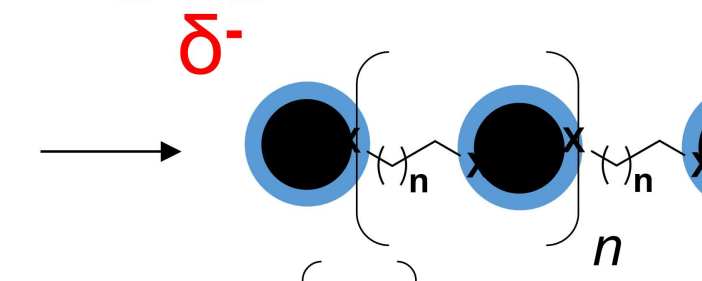
$\delta=n$ $\delta-$
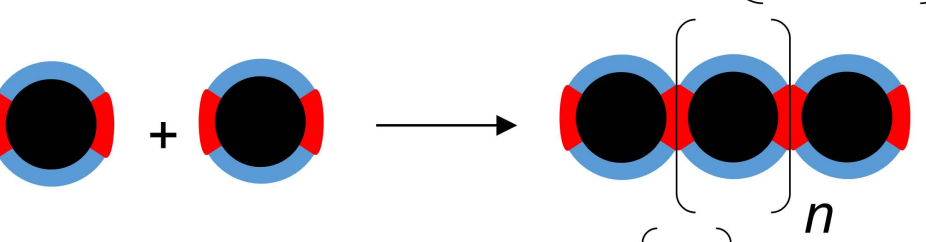
"patchy particles"

c) Dipole-directed dipole 1) electrostatic
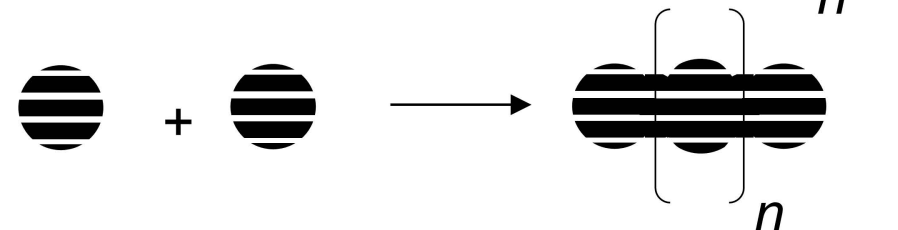

forms single crystals

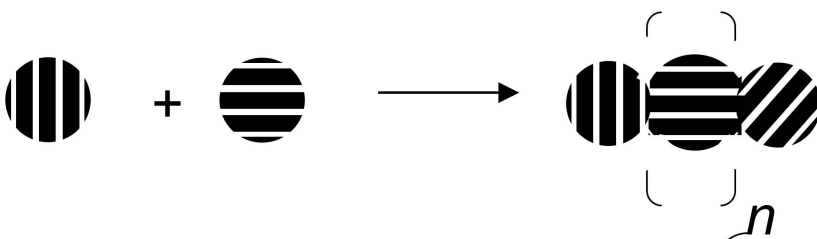
random crystal orientations

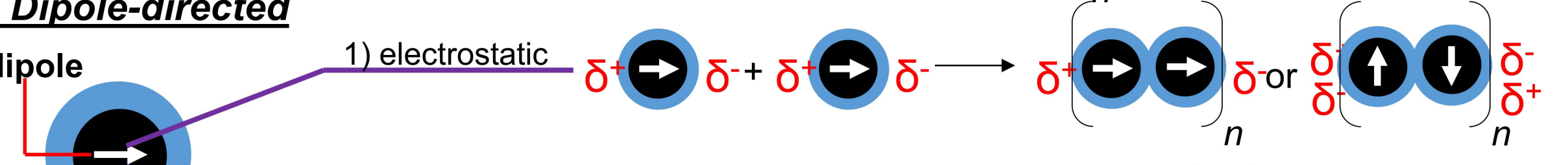

2) magnetic (spin)

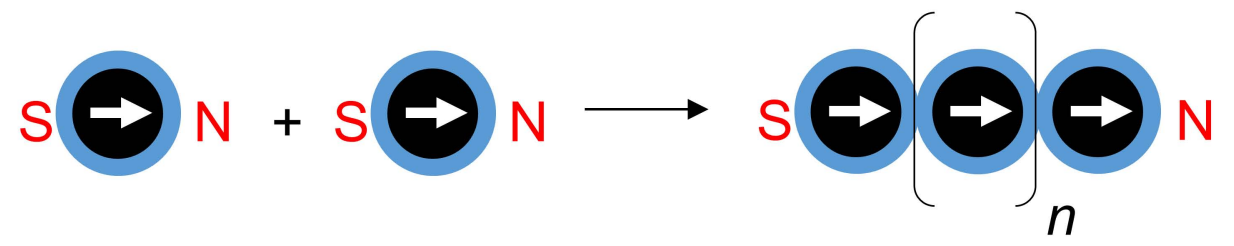

